Florida International University

FIU Digital Commons

FIU Electronic Theses and Dissertations

University Graduate School

$11-8-2019$

\title{
Sensor-Based Adaptive Control and Optimization of Lower-Limb Prosthesis.
}

Roozbeh Atri

ratri@fiu.edu

Follow this and additional works at: https://digitalcommons.fiu.edu/etd

Part of the Biomedical Commons, Controls and Control Theory Commons, and the Signal Processing Commons

\section{Recommended Citation}

Atri, Roozbeh, "Sensor-Based Adaptive Control and Optimization of Lower-Limb Prosthesis." (2019). FIU Electronic Theses and Dissertations. 4328.

https://digitalcommons.fiu.edu/etd/4328

This work is brought to you for free and open access by the University Graduate School at FIU Digital Commons. It has been accepted for inclusion in FIU Electronic Theses and Dissertations by an authorized administrator of FIU Digital Commons. For more information, please contact dcc@fiu.edu. 


\section{FLORIDA INTERNATIONAL UNIVERSITY}

Miami, Florida

\section{SENSOR-BASED ADAPTIVE CONTROL AND OPTIMIZATION OF LOWER- LIMB PROSTHESIS}

A dissertation submitted in partial fulfillment of the requirements for the degree of DOCTOR OF PHILOSOPHY

in

ELECTRICAL AND COMPUTER ENGINEERING

by

Roozbeh Atri 
To: Dean John L. Volakis

College of Engineering and Computing

This dissertation, written by Roozbeh Atri, and entitled Sensor-Based Adaptive Control and Optimization of Lower-Limb Prosthesis, having been approved in respect to style and intellectual content, is referred to you for judgment.

We have read this dissertation and recommend that it be approved.

$\begin{array}{r}\hline \text { Kang K. Yen } \\ \hline \text { Armando Barreto } \\ \hline \text { Ahmed Ibrahim } \\ \hline \text { Wei-Chiang Lin } \\ \hline \text { Ou Bai, Major Professor }\end{array}$

Date of Defense: November 8, 2019

The dissertation of Roozbeh Atri is approved.

Dean John L. Volakis College of Engineering and Computing

Andrés G. Gil

Vice President for Research and Economic Development and Dean of the University Graduate School

Florida International University, 2019 
(C) Copyright 2019 by Roozbeh Atri

All rights reserved. 


\section{DEDICATION}

To my dearest Tom and my lovely parents. 


\section{ACKNOWLEDGMENTS}

First, I would like to express gratitude to my advisor Dr Ou Bai for his patience, motivation and immense knowledge. His mentorship helped me grow both personally and professionally. He never hesitated to devote his time, regardless of day or night, to discuss the research.

I would like to thank the members of committee, Dr. Yen, Dr. Barreto, Dr Ibrahim, and Dr. Lin, for taking the time to evaluate and comment on this dissertation.

I would also like to thank Dr. Murphy, Mr. Lovegreen, Dr. Fei, and Dr Gorgey in Hunter Holmes McGuire VA Medical Center in Richmond, Virginia for their help with data collection. I will forever be grateful for the time they took out of their busy schedule to assist me with finding volunteer subjects and collect data.

A special thanks to my friends and colleagues in the Human Cyber Physical Systems (HCPS) lab, Sebastian, Rod, Masud, Tushar, Tong, Robin, and Connie for their support and help throughout various stages of the research.

A special thanks to my best friend Amin who has always been there during challenging times and found a way to help me navigate through it. My deepest gratitude to my parents and siblings. Without their support, this would not have been possible.

This research was supported by the Graduate assistantship from the Department of Electrical and Computer Engineering at Florida International University and National Science Foundation (CNS-1552163). 


\title{
ABSTRACT OF THE DISSERTATION \\ SENSOR-BASED ADAPTIVE CONTROL AND OPTIMIZATION OF LOWER- LIMB PROSTHESIS
}

\author{
by \\ Roozbeh Atri \\ Florida International University, 2019 \\ Miami, Florida \\ Professor Ou Bai, Major Professor
}

Recent developments in prosthetics have enabled the development of powered prosthetic ankles (PPA). The advent of such technologies drastically improved impaired gait by increasing balance and reducing metabolic energy consumption by providing net positive power. However, control challenges limit performance and feasibility of today's devices. With addition of sensors and motors, PPA systems should continuously make control decisions and adapt the system by manipulating control parameters of the prostheses. There are multiple challenges in optimization and control of PPAs. A prominent challenge is the objective setup of the system and calibration parameters to fit each subject. Another is whether it is possible to detect changes in intention and terrain before prosthetic use and how the system should react and adapt to it.

In the first part of this study, a model for energy expenditure was proposed using electromyogram (EMG) signals from the residual lower-limbs PPA users. The proposed model was optimized to minimize the energy expenditure. Optimization was performed using a modified Nelder-Mead approach with a Latin Hypercube sampling. Results of the 
proposed method were compared to expert values and it was shown to be a feasible alternative for tuning in a shorter time.

In the second part of the study the control challenges regarding lack of adaptivity for PPAs was investigated. The current PPA system used is enhanced with impedancecontrolled parameters that allow the system to provide different assistance. However, current systems are set to a fixed value and fail to acknowledge various terrain and intentions throughout the day. In this study, a pseudo-real-time adaptive control system was proposed to predict the changes in the gait and provide a smoother gait. The proposed control system used physiological, kinetic, and kinematic data and fused them to predict the change. The prediction was done using machine learning based methods. Results of the study showed an accuracy of up to 98.7 percent for prediction of change for four different cases. 


\section{TABLE OF CONTENTS}

CHAPTER

PAGE

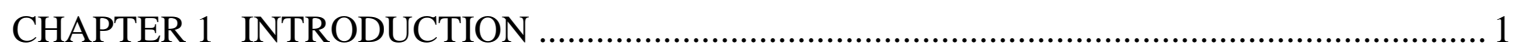

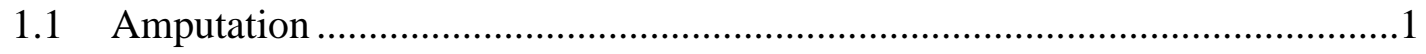

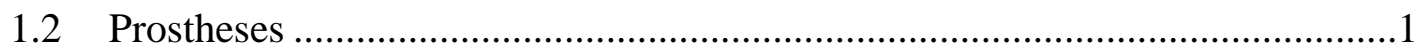

1.2.1 Passive Prostheses .........................................................................

1.2.2 Active prostheses..........................................................................

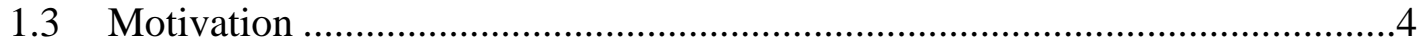

1.4 Body-area sensor network …………………….........................................

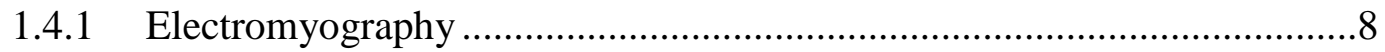

1.4.2 Inertial Measurement Unit ..................................................................12

1.4.3 Piezo-Resistive Insole Pressure Sensors …………………….................13

1.4.4 Infrared Distance Sensor ....................................................................15

1.4.5 Custom Made BASN ........................................................................16

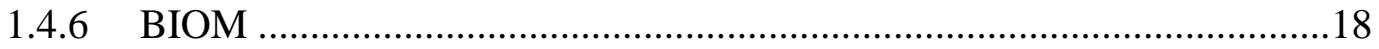

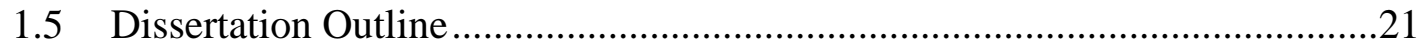

CHAPTER 2 POWERED PROSTHESES OPTIMIZATION ………………………………....2

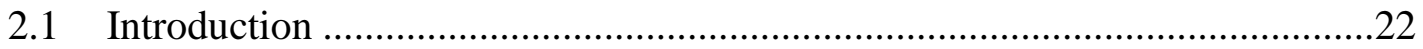

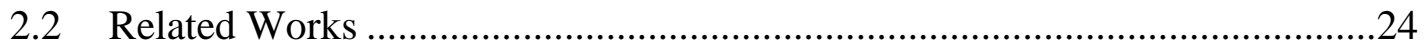

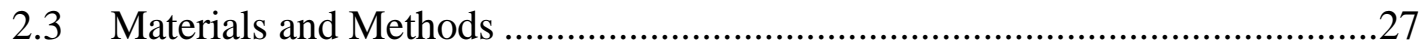

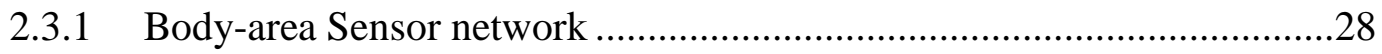

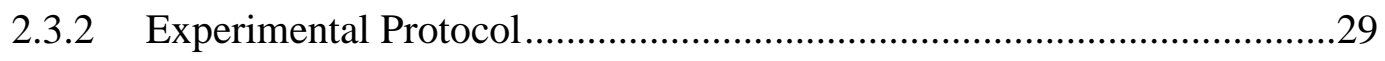

2.3.3 Energy Expenditure Estimation from EMG ..........................................31

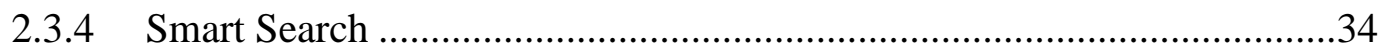

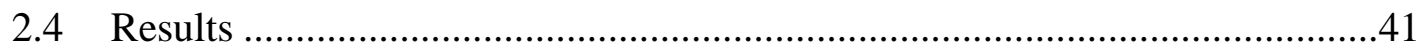

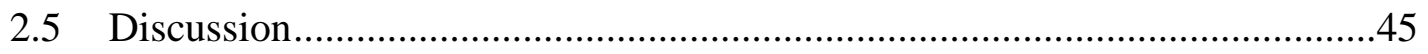

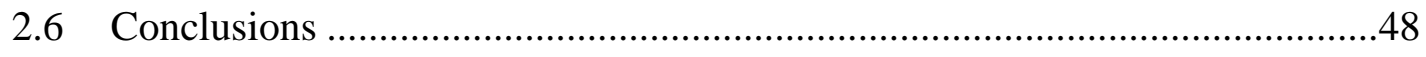

CHAPTER 3 ADAPTIVE CONTROL OF POWERED PROSTHESES .................................... 49

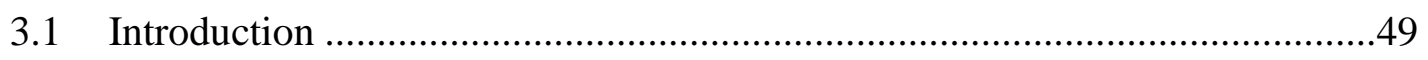

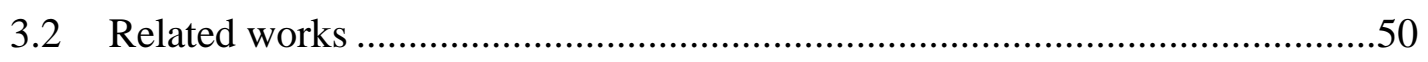

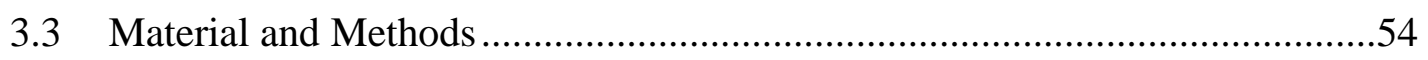




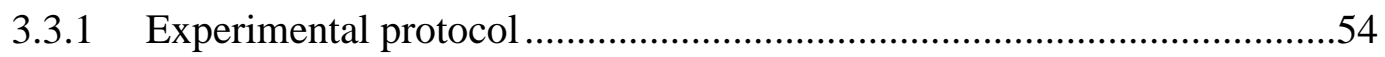

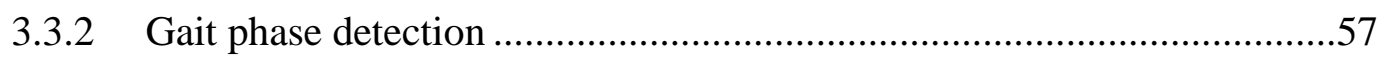

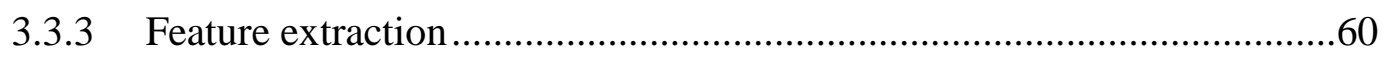

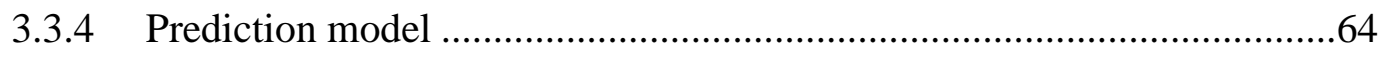

3.3.5 Pseudo real-time testing .................................................................66

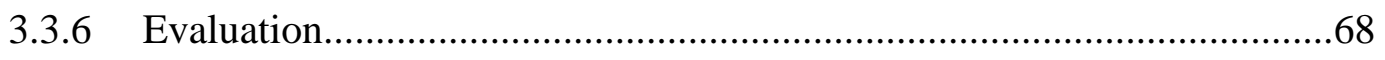

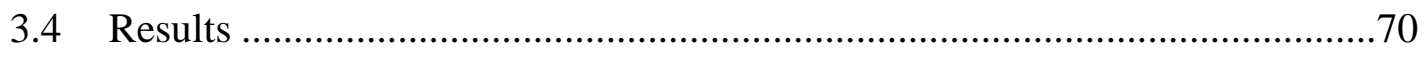

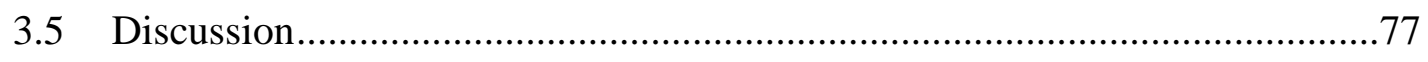

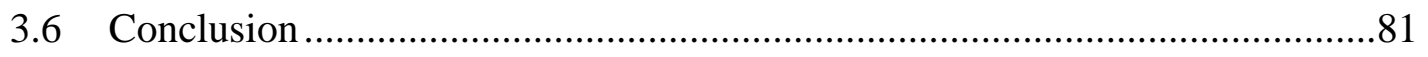

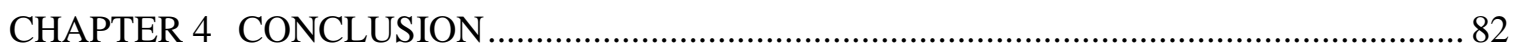

4.1 Future direction....................................................................................... 84

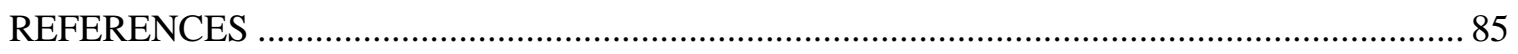

VITA 


\section{LIST OF TABLES}

TABLE

PAGE

Table 1. Results of the EMG-based smart search to tune PPA..................................42

Table 2. Review of the related works for PPA control ...........................................51

Table 3. Physical features of the prosthetic control subjects. ...................................54

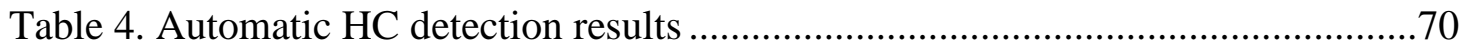

Table 5. Selected features using feature importance of extended tree classifier. .........71

Table 6. Accuracy, Sensitivity and, Specificity measures for change and terrain prediction for level terrain, stair ascent (SU), stair descent (SD), and turn (T).. 77 


\section{LIST OF FIGURES}

FIGURE

PAGE

Figure 1. Prosthetic fitting process. from left to right the parts are the molding, filled mold, clear test socket, final fiber carbon socket, and the liner. 3

Figure 2. lower limb muscle locations and names [38].......................................11

Figure 3. IMU sensor placement on the intact ankle, both hips and knees.................13

Figure 4. Gait phases during one gait cycle 14

Figure 5. Ground Reaction Force (GRF) system including three piezo-resistive pressure sensors located in toe, midfoot, and heel.

Figure 6. custom-made Body Area Sensor Networks (BASN) designed for the study. From left to right the sensors are Infrared distance sensor, ground reaction forces sensor, electromyogram sensor. Each EMG sensor is capable of recording to channels of myographic signals and it is embedded with a 9 degree of freedom Inertial Measurement Unit sensors.

Figure 7. Design BIOM powered prosthetic ankle [1].

Figure 8. The mechanical model for the series elastic actuator (SEA) for the BIOM ankle [1].

Figure 9. Data collection setup using a treadmill and a harness to prevent fall. (a) Amputee subject on the treadmill with a protective harness on (b) electrode placement on TA and GL muscles on the intact limb. 30

Figure 10. 10 channels of EMG from lower limb of subject 1 while operating PPA with zero percent power and zero percent stiffness for five steps. The pitch signals depicted on top was used to detect each step and it was used to partition EMG signals to each step. Two steps detected from the pitch signals are highlighted.. 32

Figure 11. Grids of the estimated metabolic energy model using a 10-channel EMG sensor for various combinations of the stiffness and power parameters for three bent-knee and three transfemoral amputee subjects.

Figure 12. Error for smart search using the GA method in various population sizes. GA method was investigated using various initial population sizes to identify the smallest population size needed to converge to the global minima faster. The results are evaluated using an error measure which is the distance of the result of optimization from the target value. 44

Figure 13. Error for smart search using NM and LHS methods in various vertices and random samples. (a) The distance from the target value for various simplexes. 
(b) The distance for the NM optimization using LHS with a various number of intervals to limit the search. 45

Figure 14. Transition to different terrain while using PPA. (a) The subject takes three steps on level ground and transitions to stair ascend leading with intact limb. (b) Subject walks on level ground and transitions to stair descend leading with the intact limb. (c) Subject walks toward the stairs but instead of ascending, the stairs turn and comes back. 52

Figure 15. Simulated prosthetic user (a), Amputee subject (b)....................................55

Figure 16. Ground reaction forces recorded using insole piezo-resistive sensors inside prosthetic shoes and detected heel contact times. 59

Figure 17. EMG signal from Soleus before and after motion artefact removal of subject 1

Figure 18. A general overview of gradient boosted trees. .66

Figure 19. Flowchart for the pseudo-real-time terrain mode prediction and adaptive control for powered prosthetic ankle optimization. 67

Figure 20. Feature selection results

Figure 21. A sample of GRF and EMG signals with detected HCs for two level terrain gait cycles followed by a stair ascend step. The transition and one level terrain sample are highlighted in blue. 72

Figure 22. Distribution of selected features for four different classes investigated in this study. (a) IR distance reading at $\mathrm{HC}$ for subject 4; (b) maximum for gyro signal in z-axis on the intact hip of subject 2; (c) minimum for accelerometer in y-axis on amputee's hip for subject 1; (d) RMS for intact Soleus for subject 3. 73

Figure 23. Pseudo-real-time testing results for detection of the change in locomotion mode.

Figure 24. Pseudo-real-time testing results for prediction of stair ascent.....................75

Figure 25. Pseudo-real-time testing results for prediction of stair descent...................75

Figure 26. Pseudo-real-time testing results for prediction of turn. ..............................76

Figure 27. Confusion matrix for pseudo real-time testing of terrain prediction on four subjects. 


\section{LIST OF ACRONYMS AND ABBREVIATIONS}

\begin{tabular}{|c|c|}
\hline TF & Transfemoral \\
\hline PPA & Powered Prosthetic Ankle \\
\hline BSAN & Body Area Sensor Network \\
\hline EMG & Electromyography \\
\hline IMU & Inertial Measurement Units \\
\hline PS & Piezo-Resistive Insole Pressure Sensors \\
\hline IR & Infrared Distance Sensor \\
\hline SENIAM & Surface Electromyography for the Non-Invasive Assessment of Muscles \\
\hline Sol & Soleus \\
\hline TA & Tibialis Anterior \\
\hline GL & Gastrocnemius Lateralis \\
\hline VL & Vastus Lasteralis \\
\hline RF & Rectus Femoris \\
\hline GMed & Gluteus Medialis \\
\hline $\mathrm{BF}$ & Biceps Femoris \\
\hline $\mathrm{MCU}$ & Microcontroller Unit \\
\hline GRF & Ground reaction forces \\
\hline ToF & Time-of-Flight \\
\hline $\mathrm{CPO}$ & Certified Prosthetist and Orthoptist \\
\hline ES & Exhaustive Search \\
\hline GA & Genetic Algorithm \\
\hline NM & Nelder-Mead \\
\hline
\end{tabular}




\begin{tabular}{|c|c|}
\hline LHS & Latin Hypercube Sampling \\
\hline GM & Gluteus Medius \\
\hline BK & Bent-Knee \\
\hline IRB & Institutional Board Review \\
\hline MAV & Mean Absolute Value \\
\hline $\mathrm{HC}$ & Heel Contact \\
\hline $\mathrm{HO}$ & Heel Off \\
\hline Var & Variance \\
\hline LogDet & Log Detector \\
\hline WL & Wavelength Length \\
\hline WAmp & Willison Amplitude \\
\hline SSC & Slope Sign Change \\
\hline ETC & Extra Tree Classifier \\
\hline SVM & support vector machine \\
\hline RBF & radial basis function \\
\hline GBT & Gradient Boosted Tree \\
\hline ANN & Artificial Neural networks \\
\hline BFGS & Broyden-Fletcher-Goldfarb-Shanno \\
\hline L-BFGS & limited-memory Broyden-Fletcher-Goldfarb-Shanno \\
\hline
\end{tabular}




\section{CHAPTER 1 INTRODUCTION}

\subsection{Amputation}

Amputation is the surgical removal of a limb where the main cause of such operations are vascular disease (54\%) including diabetes and peripheral arterial disease, trauma (45\%), and cancer (less than 2\%)[2-9]. It has been estimated that there are 185,000 new amputations annually in the United States and the amputee population is estimated to be 2 million [10, 11]. Below-knee amputations are the most common amputations, representing $71 \%$ of dysvascular amputations; there is a $47 \%$ expected increase in below-knee amputations from 1995-2020[12].

There are two types of leg amputations: Transtibial and Transfemoral. Transtibial is also known as below the knee amputation and it is performed between the ankle and the knee. Transfemoral amputation is done above the knee. Amputated limb needs to be given time for healing prior to prosthesis use.

\subsection{Prostheses}

People that go through leg amputation use prosthetic limbs to replace the lost limb. Following the recovery and healing from the amputation surgery, patients attend physical therapy and start using a temporary prosthesis. The prosthesis can be customized to fit and suit various patients. Once the limb is fully healed and can bear pressure, the prosthetic clinician takes a cast and builds a custom socket to minimize pressure and abrasion.

Using the prostheses amputees are able to gain some independence and ability to walk. However, they face many challenges such as higher metabolic energy expenditure, sores, lack of balance and symmetry, and difficulty on uneven terrains. Conventionally the 
prostheses are passive but recently there have been several powered prosthetic systems developed.

\subsubsection{Passive Prostheses}

Conventional prostheses mimic the appearance of the lost limb and provide some sort of functionality. Amputees use passive prostheses to replace the lost limb and be able to initiate gait. Such prosthetic systems consist of a socket, suspension, shaft, foot and covering. The sockets are custom designed to fit the residual limb and avoid pressure and blisters on it. The socket is connected to the prostheses using the suspension which holds it. The prosthesis is connected to the foot using the shaft and usually a cosmetic cover is used to mimic the appearance. Figure 1 shows the passive prostheses system and the molding process for the socket. However, the passive nature of these systems fail to provide the optimal assistance needed for normal walking and it has been shown that amputees consume more metabolic energy and the possibility of losing balance and falling is high. 


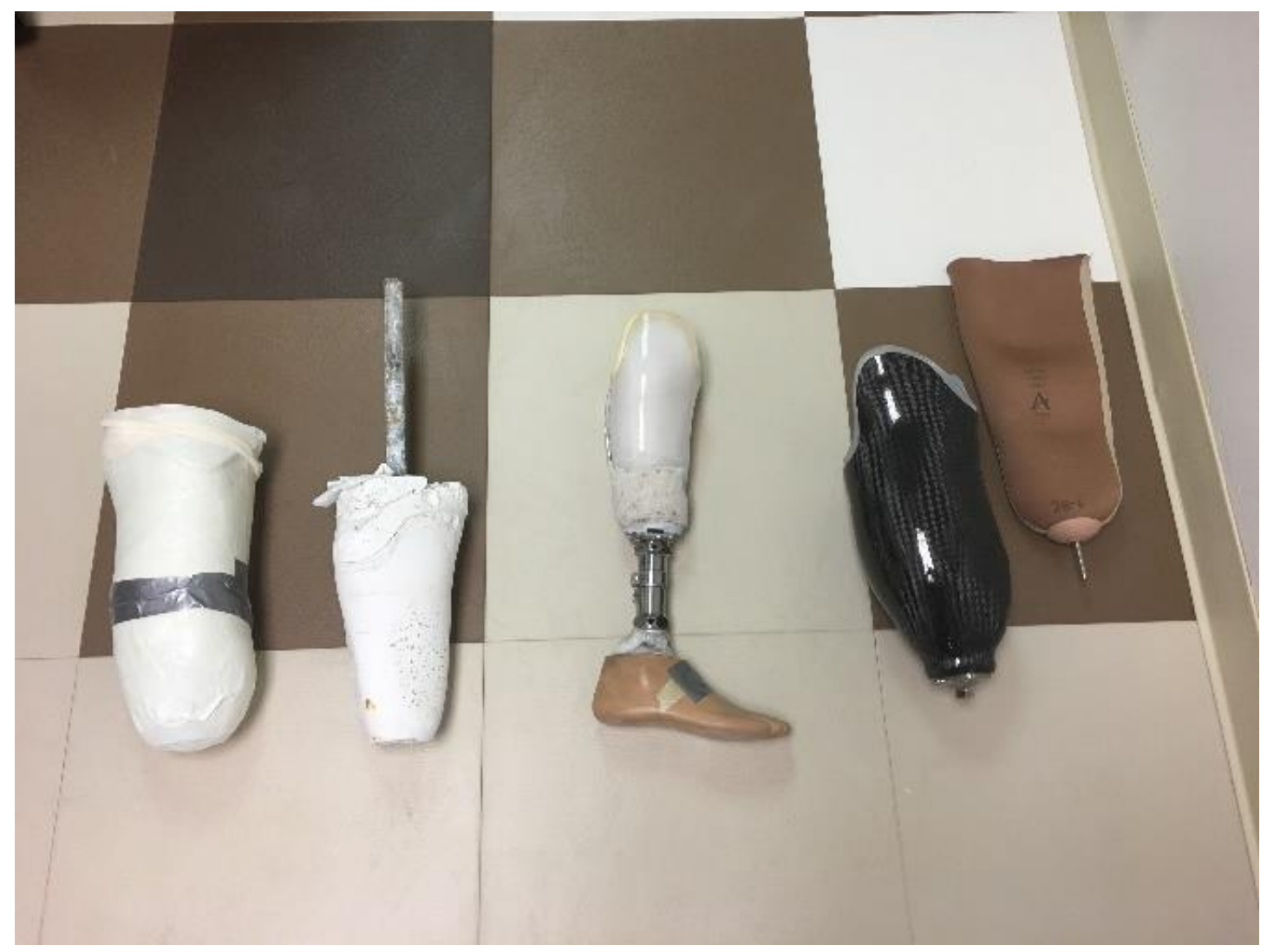

Figure 1. Prosthetic fitting process. from left to right the parts are the molding, filled mold, clear test socket, final fiber carbon socket, and the liner.

\subsubsection{Active prostheses}

During the last decade, the prostheses industry benefited from the advent of new powered technologies and with the further improvements and reliability of such technologies the use of these state-of-the-art systems became more widespread and accessible. Currently there are microprocessor control knees widely used in the industry. More recently, powered ankle-foot systems have been introduced which can be used as an intelligent system, which is capable of providing assistance with a net positive power. In this section a brief overview of powered prostheses systems will be provided and some widely studied systems will be introduced.

Earlier ideas for powered prosthetic ankles were introduced by Popovic et al. [13] and since then, several powered prosthetics have been developed.[14-20]. Early researches 
focused on automating the amputee gait and their results lead to the development of quasipassive ankle-foot prosthetics [21-23]. Quasi-passive systems use active damping and springs to passively store energy. This stored energy provides automatic adaptation to the ground surface [21, 24, 25]. Holmberg from Halmstad University [16] introduced a prosthetic ankle that automatically adjusted according to the ground angle using accelerometer data. Later, Bedard from Halmstad University as well, [14] made an actuated leg prosthesis for above-knee amputees. However, these systems lacked an actuator to provide active assistance. Goldfarb and their group from Vanderbilt University Nashville $[18,26]$ designed and controlled a pneumatically actuated transfemoral (TF) prosthesis with powered knee and ankle joints that introduced active assistance. Huang and their group from the University of Rhode Island Kingston [17] prototyped a smart prosthetic using a redundant actuator concept which enabled the system to partially function when the prosthesis loses power. Bellman et al [27] from Arizona State University also developed a system called SPARKy (Spring Ankle with Regenerative Kinetics) which had two degrees of freedom [27]. A breakthrough was made by Grabowski and Herr from Massachusetts Institute of Technology and Center for Restorative and Regenerative Medicine where, they developed a bionic prosthesis that emulates the function of the biological ankle during level walking [15, 19, 28-30]. The bionic prosthesis provided net positive work on a range of walking speeds and they showed improvement in metabolic energy expenditure and biomechanical pattern of amputee gait.

\subsection{Motivation}

Recent developments in prosthetics have enabled the development of powered prosthetic ankle (PPA) such as knees and ankles. The advent of such technologies 
drastically improved impaired gait by increasing balance, reducing metabolic energy consumption and pressure on the residual limbs by providing net positive power [15]. However, control challenges limit the performance and feasibility of today's devices. With the addition of sensors and motors, the system should continuously make control decisions and adapt the system by manipulating control parameters of the prostheses. Powered prosthetic limbs have impedance-controlled parameters such as the power of the motor and stiffness of the ankle. Having impedance-controlled parameters introduce further freedom to control the leg and ability to adapt to different intentions. To elicit the best performance of such technologies it is necessary to have a real-time prediction for the terrain type and the intention. Having an adaptive intention aware control system would help powered prosthetic systems to provide optimal assistance to amputees. Even though there have been some emerging technologies regarding the intention detection, currently, such prosthetic systems are being used with fixed control parameters, lacking the integration of the intention. The lack of intention prediction and adaptations introduces major shortcomings for the prosthetic leg when used in daily life.

The question of how assistive robots like prosthetic limbs should respond to users intent or change in the terrain and act in synchronization with the user is still open. Furthermore, there is the question regarding how early the intention should be detected and in what phase of the gait the control system should adapt the prosthetic leg. The intention detection can be done using various signals obtained from various sensors such as built-in kinetic, kinematic and physiological sensors that are placed on residual limbs. However, the main challenge is the timing of intention detection to have a reliable control system. The control system should be able to accurately predict the intention or the terrain prior to the swing 
phase on the prosthetic leg. Failure to correctly predict the intention would cause the system to use the wrong setup for the leg, which might lead to user stumbling, falling or dragging the prostheses. In addition, the susceptibility of such data to noise introduces more unreliability to the control system. The current methods have shown the possibility of using such systems to detect the intention, but the challenge in prediction has not been addressed. Major questions that require investigation for PPA optimization and control are listed as follows:

Question (1): How can PPA systems be optimized to provide objectively efficient assistance to users?

Question: (2): Is it possible to accurately predict a change in the terrain and implement a real-time adaptive control system?

The challenges regarding PPA optimization and control arise from three perspectives. On the one hand, the question of modelling the outcome of PPA assistance requires investigation. Ability to address the assistance parameter setup based on evidence rather than subjective experience will allow the PPA systems to provide objectively optimal support to the impaired gait and maintain lower metabolic energy expenditure. On the other hand, there is the challenge of predicting the changes in the terrain before PPA assistance and adjusting the system to the intention. This will ensure that the PPA system will be set up to the correct assistance mode and provide a smooth transition between various terrains and prevent loss of balance and further injury.

These challenges lead us to explore how the prosthetic control system should adapt to various intentions and terrains to improve the PPA performance and investigate real-time 
classification algorithms using various sensors as an input to predict the intention and develop an adaptive control system.

In this study, an evidence-based tuning method was developed for PPA tuning. The tuning algorithm used an optimization method to find the best tuning parameters faster. Results of tuning compared to expert values and it was shown to be feasible. In addition, a framework in which the combination of various sensors and signal processing techniques are employed to predict the intention, based on kinetic, kinematic and neurological feedback was developed. The prediction was done before prosthetic assistance with a supervised learning technique. The results of the study showed an accurate prediction performance. The results of this study show the feasibility of implementing a smart terrain prediction system for PPAs using physiological, kinetic and kinematic feedback from the residual limbs and have adaptive assistance for amputee gait.

\subsection{Body-area sensor network}

The rapid growth of physiological, kinetic, kinematic sensors, low-power circuits and wireless communication technologies allowed body area sensor networks (BASN) to go mainstream and available for a wide range of applications[31]. Body area sensor network is a wireless network of wearable computing devices [32-34]. BASN is an interdisciplinary area that allows inexpensive and continuous monitoring and can be used for optimization and control of PPAs.

The current study relies on the use of BASN to achieve the optimization and control goal for powered prosthesis systems and improve their applicability and reliability. Various sensors are employed to collect information from subjects, and they were used to develop a control system for PPA. The sensors used in this study are Electromyography (EMG), 
Inertial Measurement Units (IMU), Piezo-Resistive Insole Pressure Sensors (PS), Infrared Distance Sensor (IR). In this section, the sensors used in the study will be introduced and in the end, the custom made wireless BASN system for each information is going to be introduced.

\subsubsection{Electromyography}

Physiological processes are complex phenomena, and most physiological processes are accompanied by or manifest themselves as signals that reflect their nature and activities [35]. Electromyography is an experimental technique concerned with the development, recording and analysis of myoelectric signals. Myoelectric signals are formed by physiological variations in the state of muscle fiber membranes [36]. Skeletal muscles are made up of collections of motor unites which is the smallest muscle unit that can be activated by volitional effort [35].

There is widespread use of EMG in various application such as medical research, rehabilitation, ergonomics and sports sciences and it is proven to be an established evaluation tool. EMGs allow a direct evaluation of the muscle where it allows to measure the muscle activity.

EMG signals are noisy in nature and a raw recording is contaminated with noise. It is very important to understand the noise and remove it from the underlying EMG activity. EMG signals range between \pm 5000 microvolts and the frequency components are within 6 to $500 \mathrm{~Hz}$, showing most frequency power between 20 and $150 \mathrm{~Hz}$ [36]. Thus, it is important to consider the sampling frequency in the technical design to avoid losing information. Based on the Theorem of Nyquist and the fact that EMG bandpass can be as 
high as $500 \mathrm{~Hz}$, it is suggested to have at least $1000 \mathrm{~Hz}$ sampling frequency to avoid losing information and aliasing.

Besides the noise factor, the quality of the EMG signals is largely dependent on the skin preparation and the electrode positioning. For skin preparation, it is suggested to shave the area and clean the area using alcohol pads. Furthermore, the electrode type is chosen depending on the goal. In this study, surface electrodes are chosen to achieve a noninvasive control system. The most common type of surface electrodes is pre-gelled $\mathrm{Ag} / \mathrm{AgCl}$ electrodes, which are recommended by Surface Electromyography for the NonInvasive Assessment of Muscles (SENIAM) [37].

The focus of the study was on lower body muscles for amputee gait. Thus, the following muscle groups were investigated to develop the control system for PPA. The muscles were collected from both thighs and lower leg on both amputee and intact side. The muscles investigated are Soleus (Sol), Tibialis Anterior (TA), Gastrocnemius Lateralis (GL), Vastus Lasteralis (VL), Rectus Femoris (RF), Gluteus Medialis (GM), Biceps Femoris (BF).

The Tibialis Anterior muscle is the most medial muscle of the anterior compartment of the leg. The tibialis anterior is responsible for dorsiflexing and inverting the foot. The muscle has two origins, one being the lateral tibial condyle and the other being the upper lateral surface of the tibia, and inserts on the medial surface of the medial cuneiform and adjoining part of the base of the first metatarsal of the foot allowing the toe to be pulled up and held in a locked position. It also allows for the ankle to be inverted giving the ankle horizontal movement allowing for some cushion if the ankle were to be rolled. It is innervated by the deep peroneal nerve and acts as both an antagonist and a synergist of the 
tibialis posterior. However, the most accurate antagonist of the tibialis anterior is the peroneus longus. The tibialis anterior aides in the activities of walking, running, hiking, kicking a ball, or any activity that requires moving the leg or keeping the leg vertical. It functions to stabilize the ankle as the foot hits the ground during the contact phase of walking (eccentric contraction) and acts later to pull the foot clear of the ground during the swing phase (concentric contraction). It also functions to 'lock' the ankle, as in toe-kicking a ball, when held in an isometric contraction.

The action of the calf muscles, including the Soleus, is plantarflexion of the foot (that is, they increase the angle between the foot and the leg). They are powerful muscles and are vital in walking, running, and dancing. The soleus specifically plays an important role in maintaining standing posture; if not for its constant pull, the body would fall forward.

The rectus femoris is one of the flexors of the thigh at the hip. Results of the analysis depicted two different distribution in the energy consumption of this muscle, in two states for each leg. Vastus Lateralis extends the leg at the knee. This means that the vastus lateralis muscle straightens the leg at the knee joint such that there is an increase in the angle between the lower leg and the upper leg. It is expected that this muscle should be highly active in the gait process.

The Biceps Femoris muscle is one of three hamstring muscles that are located at the back of the thigh. These three muscles work collectively to flex the knee and extend the hip.

The Gluteus Medius muscle is partially covered, on its lower-third part, by the gluteus maximus muscle. This makes up what is commonly referred to as the buttocks. The gluteus 
medius works to provide rotation of the thigh outward from the center of the body, which enables a steady walking gait.

The location of the muscles is shown in Figure 2 and the electrode placement was done according to SENIAM recommendations [37]. It is worth noting that the lower leg muscles were investigated on the intact side as the amputee side was missing them.

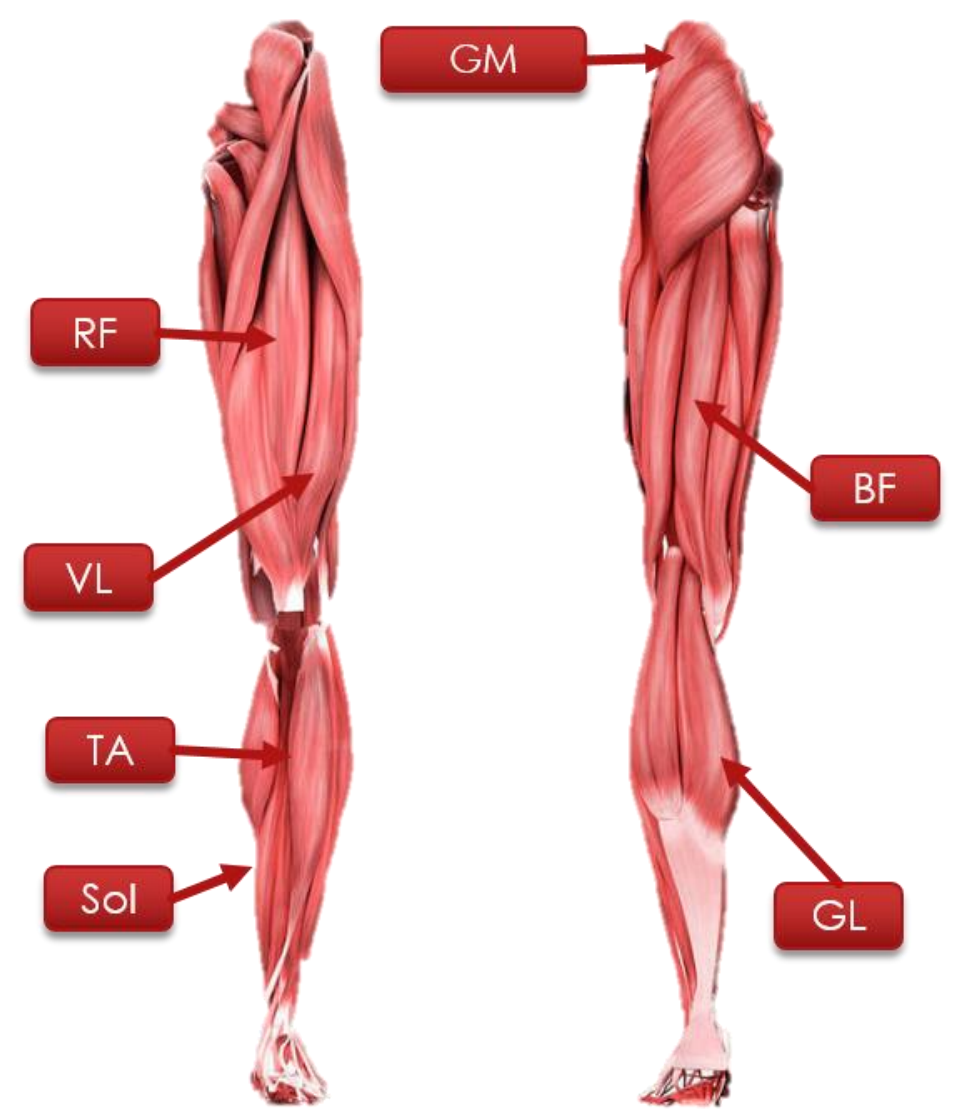

Figure 2. lower limb muscle locations and names [38].

As mentioned earlier the EMG signals tend to get contaminated with noise easily. The most important type of noise is motion artifact which happens as a result of the movement of electrodes and connecting cables. This phenomenon is more likely to happen during prosthetic gait and increasing the contamination. In this study, a bandpass filtering 
technique was implemented to remove the contamination. For this purpose, a $4^{\text {th }}$-order Butterworth filter from 20 to $450 \mathrm{~Hz}$ was used.

\subsubsection{Inertial Measurement Unit}

Inertial measurement unit is an electronic device that measures and reports a body's specific force, angular rate, and sometimes the orientation of the body, using a combination of accelerometers, gyroscopes, and sometimes magnetometers [39]. IMUs are mainly used in medical rehabilitation [40-45], robotics [46-49], and navigation [50-52]. IMU is mainly used in devices to measure velocity, orientation, and gravitational force [53]. Earlier IMUs consisted of Accelerometer and gyroscope where accelerometer measured the acceleration and gyroscope measured the angular rotation. With the advancement in technology, magnetometer was also added to IMU. The magnetometer measures the magnetic direction and can improve the gyroscope reading. Each sensor has three degrees of freedom defined as $\mathrm{x}, \mathrm{y}$, and z-axis and combined together IMU had 9 degree of freedom.

IMUs have been successfully used in gait-related studies and can be used to assess the gait characteristic. In this study, IMU sensors with 9 degrees of freedom placed on lowerbody to assess the gait characteristic and use the information for optimization and prediction process. IMU sensor collected from a custom made the sensor and multiple sensors placed on thighs, knees and ankles of amputee subjects. The mechanical signals from the IMU signals do not contain high-frequency information and they were filtered using a low-pass fourth-order Butterworth filter with bandwidth of $20 \mathrm{~Hz}$. Figure 3 shows the placement of the sensors and the axis for accelerometer and gyroscope data. 


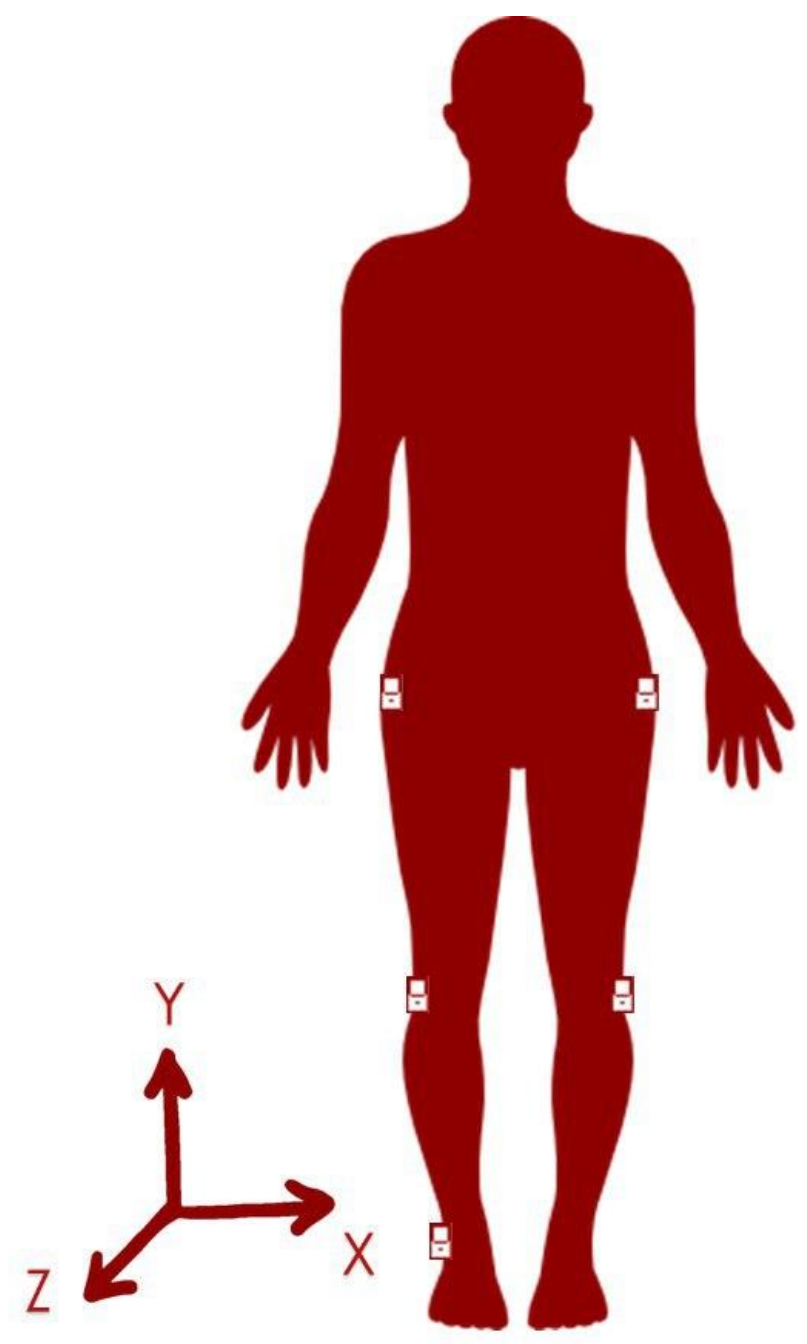

Figure 3. IMU sensor placement on the intact ankle, both hips and knees.

\subsubsection{Piezo-Resistive Insole Pressure Sensors}

The human gait is a repetitive and symmetric sequence of limb movements and gait of a normal person is a very efficient process in the means of power and velocity allowing the human to walk for a long time [54]. This optimal gait pattern changes with amputation and passive prosthetic limbs fail to provide the required assistance to compensate on the lack of symmetry and balance. PPA systems show promise in improving these problems but it is mandatory to sense and understand the normal and amputee gait and its characteristics to be able to provide an optimal control system. 
Human gait is a repetitive bipedal cycle involving steps and strides [55]. This process is consisting of two main phases; the stance phase and swing phase. The stance phase is the duration of contact between the ground and the feet and swing phase is the duration when there is no foot contact with the ground [56]. The stance phase occupies $60 \%$ of the gait cycle while the swing phase occupies only $40 \%$ of it [55]. In a more detailed classification, gait can be divided into six phases - Heel strike, Foot Flat, Mid-Stance, Heel-off, Toe-Off, Mid-Swing. Gait starts with the heel strike where the foot initially touches the ground. Following the heel strike foot flat phase is observed when the foot fully touches the ground and the other leg starts to swing. Mid-stance phase follows the heel strike when the toes for the opposite leg are off. The final phase of stance is called heel-off where the opposite leg lands on the ground and the heel starts to lose contact with the ground. Following the heel-off phase, toe-off is observed which is the first phase for the swing in the gait cycle. Finally, the swing phase is completed and the middle of the swing is called mid-swing phase. Figure 4 illustrates the normal gait for the gait phases labelled for one side.

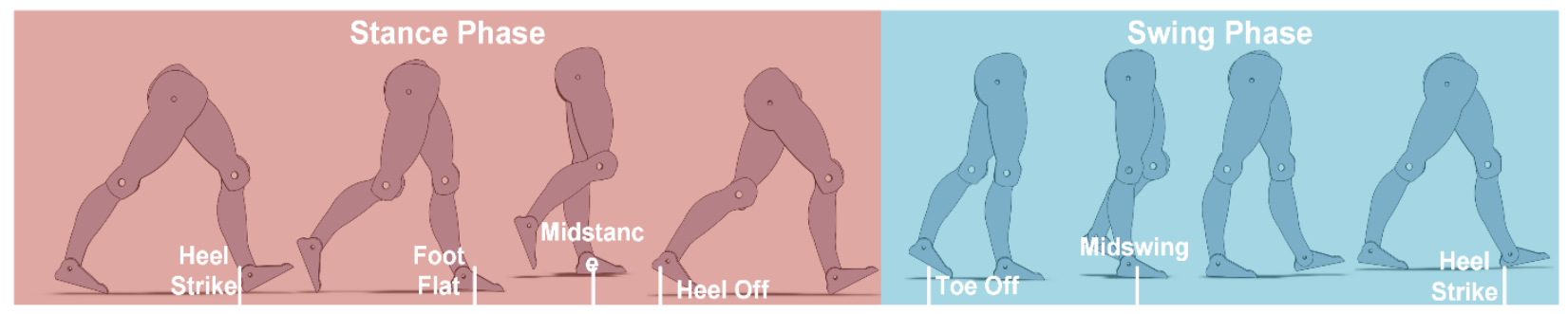

Figure 4. Gait phases during one gait cycle

Reliable control of powered prosthetic systems requires an accurate gait phase detection to prevent missed or unwanted activation. In developing the protocol, we will rely on recent works in gait phases that are pressure-based or acceleration-based [54, 57, 58]. The underlying insight is that the movement can be best observed using mechanical based 
sensors, that illustrate physical changes in the gait. By making a series of various sensors and placements on the lower-limb, we can have an extraction of the various phases in the gait that would introduce more reliability and usability for the control algorithm. For the phase estimation purpose, a piezo-resistive pressure sensing system was designed and used. The pressure system consists of three pressure sensors placed inside the sole of the shoe on the forefoot, mid-foot and heel locations. Figure 5, shows the developed insole and the pressure signals recorded for one step.

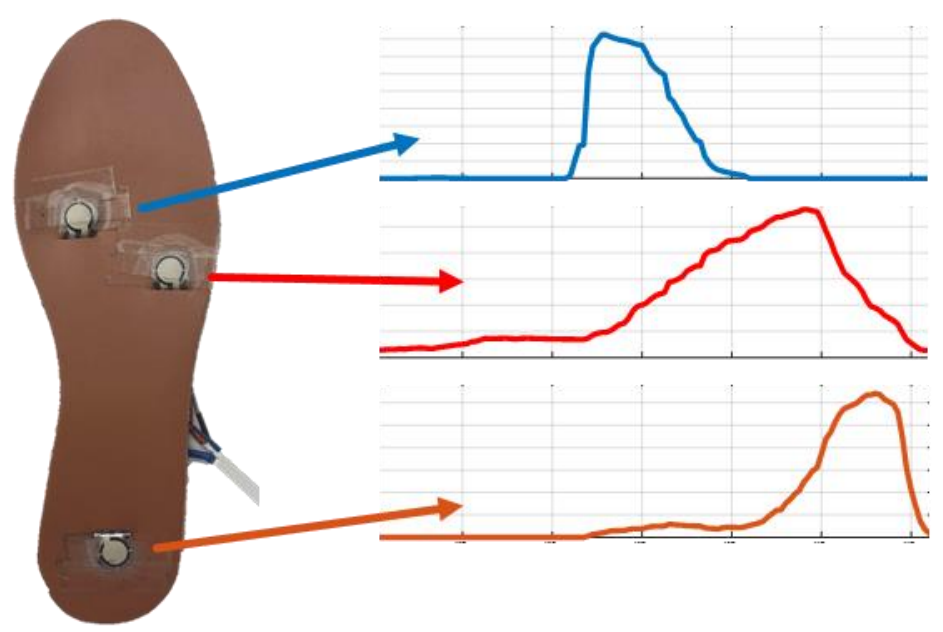

Figure 5. Ground Reaction Force (GRF) system including three piezo-resistive pressure sensors located in toe, midfoot, and heel.

\subsubsection{Infrared Distance Sensor}

While walking throughout the day, people face various obstacles and uneven terrains such as stair and ramps which require a change in the gait characteristics to maintain the symmetry and balance. To have a reliable control system for prosthetic ankles it is necessary to consider such behavior and address them. As sometimes these real-life scenarios include a physical barrier, in this study it was concluded to include a distance sensor to detect the obstacles. For this purpose, an Infrared distance sensor was developed. 
The IR sensor placed on the toe of the prosthetic foot to detect obstacles and ascension in uneven terrains.

\subsubsection{Custom Made BASN}

BASNs are wearable computing devices that consist of nodes to collect physiological and other signals. Conventionally, BSANs had a small number of data nodes and low data communication rate [59]. Recent developments in state-of-the-art technology resolved this issue and made it possible to collect many nodes of data with a higher sampling rate [5961]. For this study, various custom-made BSAN systems developed to obtain the information and process them for optimization and control of the system. The BSAN systems are wifi-based and wearable. For this purpose, CC3200 wireless MCU used.

The CC3200 SimpleLink Wi-Fi is the industry's first Wi-Fi certified single-chip microcontroller unit (MCU) with built-in Wi-Fi connectivity (ti.com/product/CC3200). Created for the Internet-of-Things (IoT), the SimpleLink CC3200 chip device is a wireless MCU that integrates a high-performance ARM Cortex-M4 MCU with a fast parallel camera interface, I2S, SD/MMC, UART, SPI, I2C, and four-channel ADC. The Wi-Fi network processor subsystem features a Wi-Fi Internet-on-a-Chip and contains an additional dedicated ARM MCU that completely offloads the applications MCU. The WiFi subsystem includes an $802.11 \mathrm{~b} / \mathrm{g} / \mathrm{n}$ radio, baseband, and MAC with a powerful crypto engine. In this study, the SimpleLink CC3200 single-chip system implemented as the highspeed data communication engine for data transmission and sensor device control [59].

IMU measurement is done using the MPU-9250 motion tracking device [62]. MPU9250 is a 9 -axis motion processing unit which is delivered in $3 \times 3 \times 1 \mathrm{~mm}$ and is the world's smallest 9-axis motion tracking sensor. This sensor is low-power with consumption 
performance of $9.3 \mu \mathrm{A}$. MPU-9250 consists of 3-axis accelerometer, 3-axis gyroscope, and 3-axis gyroscope. The configuration and data access achieved using the $\mathrm{I} 2 \mathrm{C}$ bus.

For EMG recording a high accuracy A/D converter named ADS1292 was used. ADS1292 is a 24-bit, two-channel, low-power analogue to digital converter. This A/D converter embedded with all the features commonly required for portable bio-potential signals. ADS1292 is capable of high sampling rate which makes it suitable for EMG signal recording.

Ground reaction forces (GRF) are measured using Tekscan A301 piezoresistive transducers [63]. The pressure signal amplified using the MCP6004 Op Amp. A powerpath management IC used along with the LTC 2942-1 for battery charging, voltage, and temperature measurement. GRF system compromised of an insole embedded with three piezo transducers placed on forefoot, mid-foot and heel.

For IR sensor design, VL53L0X sensor used. The VL53L0X is a new generation Timeof-Flight (ToF) laser-ranging module housed in the smallest package on the market today, providing accurate distance measurement whatever the target reflectance, unlike conventional technologies. It can measure absolute distances up to $2 \mathrm{~m}$ [64].

Figure 6 shows all the sensors used in this study. The sensors designed using USR-C322 module, which is capable of wireless connectivity. 


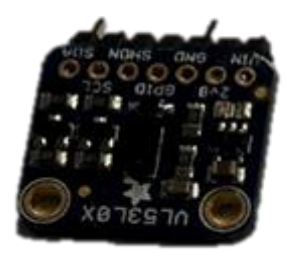

IR

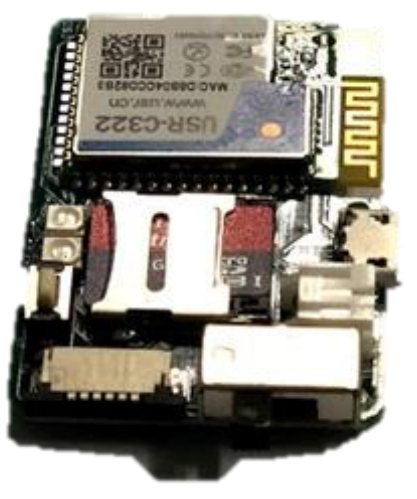

GRF

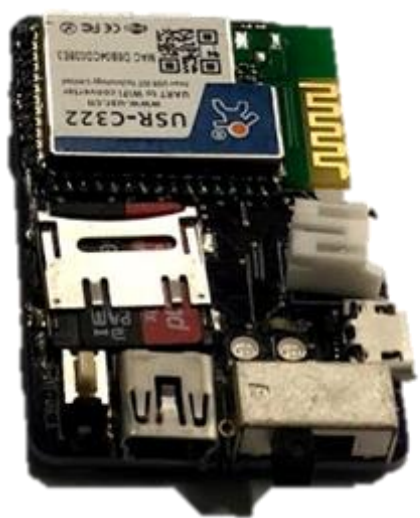

EMG

Figure 6. custom-made Body Area Sensor Networks (BASN) designed for the study. From left to right the sensors are Infrared distance sensor, ground reaction forces sensor, electromyogram sensor. Each EMG sensor is capable of recording to channels of myographic signals and it is embedded with a 9 degree of freedom Inertial Measurement Unit sensors.

\subsubsection{BIOM}

The PPA used in this study is the BIOM ankle designed by Hugh Herr and Alena Grabowski $[15,30]$. BIOM ankle is the only commercially available prosthetic ankle that provides net-positive power and it has been shown that reduces the metabolic energy expenditures and provides a better gait performance and balance.

The BIOM prosthetic attaches to the socket via a pylon and has a mass of $2 \mathrm{~kg}$. The prosthetic includes an actuator in-series with a carbon-fiber leaf spring, in parallel with a unidirectional leaf spring, and heel and forefoot leaf springs that provide elasticity [15]. A series-elastic actuator performs negative and positive work. The actuator comprises a 200 W DC brushless motor (Maxon EC-Powermax 30) and ball-screw transmission (Nook $14 \times 3 \mathrm{~mm}$ ) in series with a carbon-composite leaf spring. A $0.22 \mathrm{~kg}$ Lithium-polymer 
rechargeable battery provides energy to the motor. The prosthesis is $67 \%$ efficient; approximately $30 \mathrm{~J}$ of electrical energy produces $20 \mathrm{~J}$ of net positive work during the stance period of walking, the typical energy requirement for an $80 \mathrm{~kg}$ person walking at $1.75 \mathrm{~ms}^{-1}$ $[15,30]$. A charged battery produces $4000-5000$ steps, sufficient to walk $4-5 \mathrm{~km}$ at $1.75 \mathrm{~ms}^{-}$

${ }^{1}$ and exceeding the $3060 \pm 1890$ steps per day typically walked by an active PWA [15, 65]. Figure 7 shows the designs of the BIOM [15].
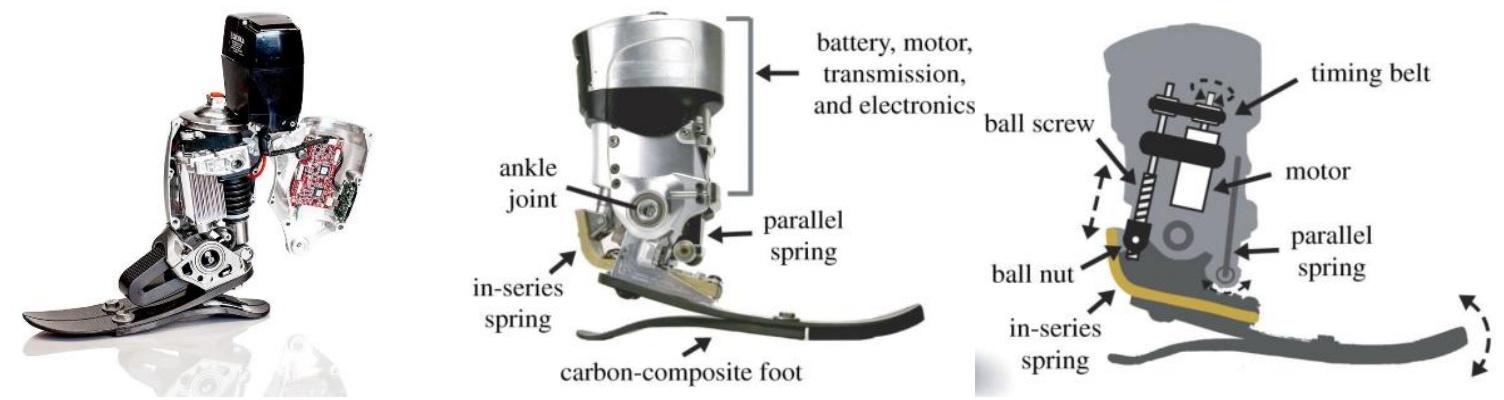

Figure 7. Design BIOM powered prosthetic ankle [1]. 
The BIOM system mechanically modelled as a series elastic actuator (SEA) which includes a motor in series with a transmission and a series spring. The model also includes a unidirectional parallel spring. Figure 8shows the mechanical model presented by Herr et al.[1].

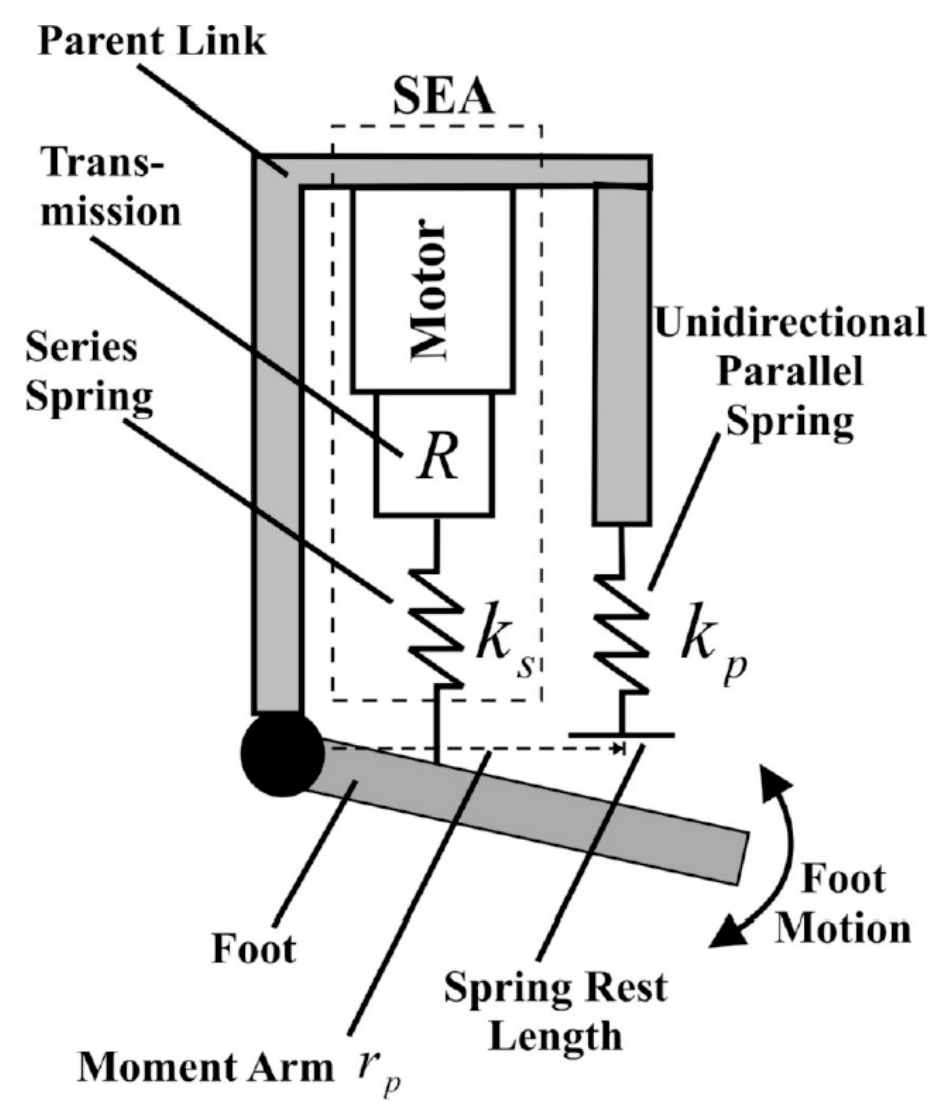

Figure 8. The mechanical model for the series elastic actuator (SEA) for the BIOM ankle [1].

Herr et al. proposed the following model for the torque output of the presented mechanical model.

$$
\begin{gathered}
M_{e} \ddot{x}+b_{e} \dot{x}=F_{e}-F_{s} . \\
F_{s}=k_{s}\left(x-r_{s} \theta\right) . \\
T_{e x t}= \begin{cases}r_{s} F_{s} & \theta<0 \\
r_{s} F_{s}+r_{p} k_{p} \theta & \theta \geq 0\end{cases}
\end{gathered}
$$


Where, $M_{e}$ is the effective mass, $b_{e}$ is the damping, $F_{e}$ is a linear motor force, $F_{s}$ is a series motor force, $T_{\text {ext }}$ is output torque of the ankle, $k_{s}$ and $k_{p}$ are series and parallel spring stiffness respectively, and $r_{s}$ and $r_{p}$ are series and parallel spring moments.

The commercially available system can be adjusted to various subjects with different physical features. The tuning process for this part is done by a certified prosthetist and orthoptist (CPO). The tuning process in conducted subjectively by relying on the expert's knowledge and the users' feedback. Following the setup, CPO chooses the best stiffness and power values for the user and the assistance is the same regardless of the intention of the user.

In the next section, a novel objective tuning method for prosthetic fitting is proposed and in chapter 4 , a terrain type prediction algorithm is developed to provide an adaptive control system for improved performance and usability of the PPA.

\subsection{Dissertation Outline}

The rest of the dissertation is organized in four parts. In the second chapter, a smartsearch algorithm developed to objectively tune the PPA system parameters. The proposed methods used an evidence-based method combined with an optimized search technique for parameter setup. Results compared to expert values and shown to be feasible alternative. Chapter three presents a pseudo-real-time terrain type prediction method is presented. The proposed method used sensor fusion method to predict the terrain type before HC of the PPA and results showed accuracy of up to $98 \%$ for the tested terrain types in real-time. Finally, chapter four will discuss the findings of this dissertation and highlight the future work needs to be done. 


\section{CHAPTER 2 POWERED PROSTHESES OPTIMIZATION}

\subsection{Introduction}

Recent improvements in prosthetics introduced powered ankles and knees to the amputee gait and the advent of such technologies brought more balance and symmetry to the users. These prosthetic systems are embedded with battery-driven mechanical motors to provide extra torque and assists the users by propelling them forward without requiring them to supplement excessive metabolic energy. The history of powered prosthesis development presented in chapter 1 . In this study, BIOM ankle which is commercially available was used.

PPA systems can be controlled and fitted to various user types with different physical features by manipulating impedance-controlled parameters. The main parameters are stiffness for the ankle and the amount of power provided by the system [66]. The stiffness parameter controls how the ankle flexes and helps users to have a smooth transition between various phases in the gait, which makes it crucial to adjust it to fit each user [67-

70]. If the stiffness was set to a low value, it might cause the user to catch himself/herself by consuming more energy to prevent falling and, if it were set to a higher value than necessary, the prosthetic gait would introduce more dissidence to the gait and undermine the effectiveness of the system. In addition, the amount of power provided by the leg needs to be adjusted as well and its value relies on the weight, height, and strength of the user. If this parameter is not set properly, it will result in under-powering or over-powering; underpowering would cause the user to drag the leg, consume more metabolic energy, and have more pressure on the residual limb, while over-powering would provide excessive push from the system and cause the user to stumble and increase the possibilities of falling. 
The setup for PPA systems is currently done by certified prosthetists and orthotists. The CPO tunes the powered system after achieving a good fit for the prosthetic socket and other equipment [71-73]. The way the tuning is done is based on the CPO's observation and the feedback that the user provides. CPO asks the amputee to walk in an assisted walkway while they change the parameters and observe the gait. The best parameter setup is achieved when the CPO is satisfied with his/her observation. This process is timeconsuming as it requires the user to walk back and forth while the $\mathrm{CPO}$ manipulates the parameters and observes their gait pattern until they are satisfied with the performance. The current methods are subjective and observation-based, and there are not many tools to evaluate and quantify the fitness of the parameters. In this study, a smart data-driven tuning method was introduced. The proposed method uses physiological data to build an evidencebased method to calibrate the parameters of the PPA system.

The results of this study showed the feasibility of using smart search for objectively tuning the powered prosthetic systems in a much shorter time with surface EMG signals from the residual limb. The results of the study found the optimal parameter setup with far less number of iterations compared to an exhaustive searching. Furthermore, the results showed that by using this method, it is possible to replace the observation-based tuning which requires trial and error for tuning with evidence-based smart searching which uses biological feedback from the amputee while the PPA is in operation.

The rest of the optimization study is organized as follows. In section 3.2, a review of previous works on PPA tuning and optimization techniques are presented. In section 3.3, materials and methods including the sensor system and the processing and optimization are introduced. In section 3.4, the results of the implementation of the proposed method are 
presented. Finally, results of the study are discussed in section 3.5 and the conclusion is presented in section 3.6.

\subsection{Related Works}

Recently, several studies have focused on systems to quantify the observation and do the tuning automatically using mechanical sensors. Wang et al [74] developed a fuzzy logic-based system using the knee angle and phase duration that can tune the control impedance for a powered knee prosthesis, resulting in increased symmetry for the nonamputee subjects. Huang et al [75] also used a powered knee with a passive ankle and developed a fuzzy-based cyber expert system using temporal symmetry, stance width, and trunk sway. Wen et al [76] also worked on powered knee prosthesis and proposed two automatic tuning strategies - parallel and sequential. The system was tested on able-bodied subjects and the results showed the possibility of using such technology to match the ideal knee profile to improve gait. All the works in this field focused on prosthetic knees and did not consider powered prosthetic ankles that transtibial amputees operate. In addition, the focus of these works was on mechanical sensors and they did not consider the biological aspect of the gait. Recent studies have shown the possibility of using electromyogram signals to control exoskeletons and foot orthosis [77-79]. Since mechanical sensors show the effect of movement after the movement was made, it was assumed that considering the biological data would provide a physiology-based model that is more robust to control and optimize the prosthetic systems. The assumption was made based on the fact that the motor movement is the result of physiological stimulation in the muscles. Our recent work focused on this assumption by using EMG signals to model amputees' gait in a physiological sense and investigated the sensitivity of different muscle groups to the 
changes in natural gait, and the results showed that users rely more on the sound leg to complete gait [80]. Later, a pilot study was conducted to tune a powered prosthetic ankle using multi-channel EMG signals using grids of various possible combinations and the results were compared to experts tuning, showing the possibility of using evidence-based on physiology for automatic tuning [81]. It is hypothesized that the use of EMG signals would provide a direct observation from the amputee gait itself and can be used to model the metabolic energy expenditure, whereas mechanical sensors are unable to provide direct information about energy expenditure.

However, the proposed method relied on data collection from a limited combination of parameters, where a number of possible combinations were collected offline and a grid of stiffness and power parameters was presented. Since each parameter can vary from 0 to 100 percent, there are many possible combinations, but only a limited number of parameter combinations were considered. This limitation also constrained the resolution of the search for each parameter. To have higher resolution, a smaller-sized grid made up of more data is required, which would be highly time-consuming and not practical. The purpose of this study was to explore data-driven optimization methods to achieve a smart tuning in a short time using physiological data acquired from wireless EMG sensors. This study investigated the use of heuristic and numerical optimization methods to explore a better approach to optimize the parameter setup for PPA and converge to the optimal parameter combination without having to go through the exhaustive search. As the state-of-the-art is lacking a model for amputee gait using EMG signals, a data-driven optimization approach was introduced to be able to calibrate the parameters solely based on the observation. 
In this study, a smart searching method was explored to find the best possible setup for the PPA using biological data collected wirelessly using EMG. The smart search was achieved by a data-driven approach using multiple algorithms to investigate the speed and convergence of the methods. The most common way to search through the parameters is doing an exhaustive search (ES) where all of the possible combinations of the parameters are explored and, based on the performance of the user in each combination, the best value is chosen. Even though looking through all possible combinations would result in an accurate tuning, this approach is time-consuming since there are thousands of combinations, and would not serve as a practical replacement for current methods. To overcome the time constraint of ES, we employ the Genetic Algorithm (GA) which is the most common heuristic optimization technique which has been used in various applications such as path planning, image processing, real-time systems, etc. [82]. GA has shown to be a very effective and accurate approach for optimization. Genetic Algorithm is a heuristic method that is inspired by the process of natural selection to generate the optimized solution. Genetic algorithms are often viewed as function optimizers, although the range of problems to which genetic algorithms have been applied is quite broad [83]. The implementation of GA starts with a population of random chromosomes and the population is evaluated using a fitness function. Based on the fitness, members of the population are assigned reproductive opportunity to find a better solution [83]. Using the goodness of the members, parent chromosomes are selected for reproduction and, using a mutation technique, the next population gets generated and evaluated. This process is repeated until the user-defined stopping criteria is achieved. Stopping criteria was defined as the change in the best state in the current population and the best state in the previous iteration. 
However, since it uses a population-based optimization, which imitates the evolution theorem and updates the members of the population on every iteration, it might also require an unpractical number of trials to do tuning.

In this study, we also investigated the use of Nelder-Mead (NM) simplex method to have a more optimal search and find the best parameter combinations for PPA calibration. The Nelder-Mead simplex algorithm is a very powerful local descent algorithm, making no use of the objective function derivatives. In NM, through a sequence of elementary geometric transformations (reflection, contraction, expansion and multi-contraction), the initial simplex moves, expands, or contracts [84]. In addition, a modified NM method is introduced here by the addition of the Latin Hypercube Sampling (LHS) to limit the search area for improving the optimization and finding the optimal parameters more accurately

and faster. LSH uses a stratified sampling scheme to improve the coverage of multidimensional input space and has been used in various computer models [85]. Using LHS, the conventional NM simplex method was modified to limit the searching area and perform accurate tuning with fewer iterations.

\subsection{Materials and Methods}

In this section, the materials and methods used in this study are presented in four subsections. First, wireless body-area sensor network used to collect data is introduced. Second, the experimental in-lab protocol used to collect data using the wireless EMG sensors is explained. Third, the signal processing method that was used to extract an estimate of the energy expenditure from muscles is presented. In the last section, various 
heuristic and numerical methods that were used to optimize the gait using EMG sensors are explained.

\subsubsection{Body-area Sensor network}

This study employed custom-made EMG body-area sensor network to collect physiological signals from the residual limbs of transfemoral amputees for energy expenditure estimation and subsequently for smart automatic tuning application on PPAs. EMG signals were collected wirelessly using custom-made sensors and multi-channel EMG data was collected while users operated a PPA. The self-designed prototype is lightweight, low-power consumption, battery-powered and wireless-enabled CyberSens for EMG recording using an ADS-1292 chip. The ADS-1292 module is a low-cost, lownoise 24-bit analogue front-end biopotential measurement system recently distributed by Texas Instruments (Dallas, TX). Using an ADS-1292 module greatly reduced the cost of the CyberSens while maintaining high-quality amplification. A 32-bit MPU (CC3200) with built-in Wi-Fi connectivity was employed in the CyberSens for on-board real-time signal processing and data transmission. This self-developed CyberSens provided high-precision EMG signal with a less than $0.8 \mu \mathrm{V}$ peak-to-peak noise. The custom-made CyberSens was designed to provide seamless recording and transmission of two channels of 24-bit EMG signal with the sampling rate up to $8000 \mathrm{~Hz}$. The range of the wireless transmission can reach about 100 feet in an open space. Furthermore, an IMU sensor was also embedded in the CyberSens. An MPU-9250 (Gyro, Accelerometer, Magnetometer) MEMS motion tracking chip device (InvenSense, San Jose, CA) was employed. This chip provides a userprogrammable gyro full-scale range from \pm 250 , to $\pm 2000^{\circ} / \mathrm{sec}$ and a user-programmable 
accelerometer full-scale range from $\pm 2 \mathrm{~g}$, to $\pm 16 \mathrm{~g}$, which meets the requirement for studying human locomotion.

EMG is an experimental technique concerned with the development, recording, and analysis of myoelectric signals. Myoelectric signals are formed by physiological variations in the state of muscle fiber membranes [86]. The EMG signal provides a window on the motor as well as on its controller [87]. The EMG data were collected from ten muscles total using surface $\mathrm{Ag} / \mathrm{AgCl}$ electrodes from both limbs. The electrode placement was done using the recommendations of Surface EMG for Non-Invasive Assessment of Muscles (SENIAM) [88]. The following muscles were used in this study: Tibialis Anterior (TA), Gastrocnemius Lateralis (GL), Vastus Lateralis (VL), Rectus Femoris (RF), Biceps Femoris (BF), Gluteus Medius (GMed). The first two muscles (TA, GL) were collected from the intact limb and the other four muscles were collected from both limbs. The data was collected wirelessly using the MATLAB platform and 5 in-lab designed sensors with the sampling frequency of $1000 \mathrm{~Hz}$.

\subsubsection{Experimental Protocol}

Subjects of this study consisted of three non-amputee subjects in a bent-knee (BK) configuration and three TF amputees. Non-amputee subjects operated the leg in a bent knee position to simulate prosthetic gait. Figure 9 shows a TF amputee and electrode placement for one wireless sensor with two channels of EMG signal. Subjects operated the leg on a treadmill at a self-chosen comfortable walking speed. A harness was used to prevent falling for the subjects. The study was approved by the Institutional Board Review (IRB) in Florida International University and Hunter Holmes McGuire Veterans Administration Medical Center, and signed consent was obtained from the subjects prior to data collection. 
Empower Ankle (Ottobock, Duderstadt, Germany) that was developed by Herr et. al. [89] was used in the study. The main parameters for this ankle are the stiffness and power parameters. Each parameter varies from 0 to 100 percent with a resolution of 1 percent. In this study, the power was limited to 50 percent to avoid over-powering and falling. Subjects were asked to walk on the treadmill for 10 steps and various parameter combination were recorded to be studied offline. The stiffness parameter was varied from 0 to 100 percent with steps of 10 percent and power was varied from 0 to 50 percent with steps of 5 percent. In total, 121 various parameter combinations were collected in the recording. The collected data were analyzed offline to find the best parameter combination and various smart search methods were presented and tested to show the possibility of tuning the PPA automatically and faster.

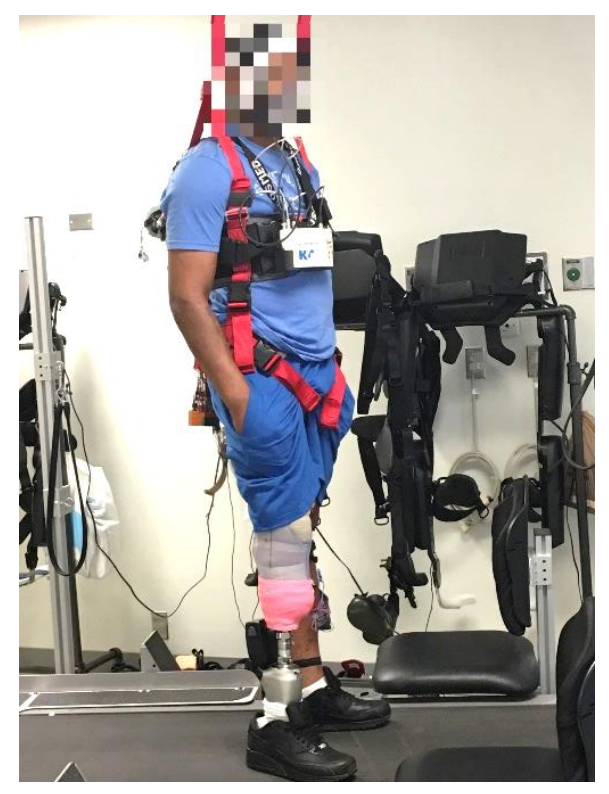

(a)

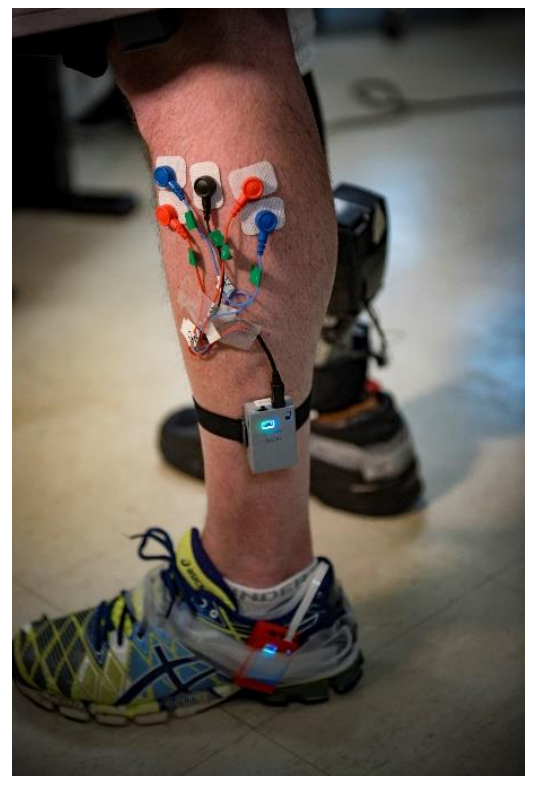

(b)

Figure 9. Data collection setup using a treadmill and a harness to prevent fall. (a) Amputee subject on the treadmill with a protective harness on (b) electrode placement on TA and GL muscles on the intact limb. 


\subsubsection{Energy Expenditure Estimation from EMG}

EMG signals are non-stationary and noisy in nature, leading to complications in the comprehension of underlying information. In addition, EMGs are highly susceptible to noise and are contaminated with a baseline drift, which depends on many factors such as quality of the EMG amplifier, the environment noise, and the electrode movement [90]. The baseline drift lies in the lower frequencies and the main EMG information is limited to the bandwidth of $20-500 \mathrm{~Hz}$ [91]. To remove the baseline and high-frequency noise, signals were filtered using a fourth-order Butterworth bandpass filter with cutoff frequencies of $20-500 \mathrm{~Hz}$.

Furthermore, gait phases were added to present an accurate phase-dependent model. Gait phases are detected using the IMU data that was incorporated in the sensor alongside EMG and recorded simultaneously. Human gait is a bipedal cycle, consisting of two phases - the stance phase and swing phase. The stance phase is the duration of contact between the ground and the feet and swing phase is duration where there is no contact between the ground and the feet [56]. IMU signals collected on the ankle are used to estimate these phases. For this purpose, the accelerometer, gyroscope and magnetometer data were collected using the 9-axis motion processing unit (MPU-9250) and by real-time on-board processing pitch signal calculated and recorded. Using a threshold and peak detection algorithm, the timing for each step was detected and used to automatically partition EMG signals into each step. Figure 10, illustrates the pitch signal on top and two of the steps that have been detected are highlighted. 


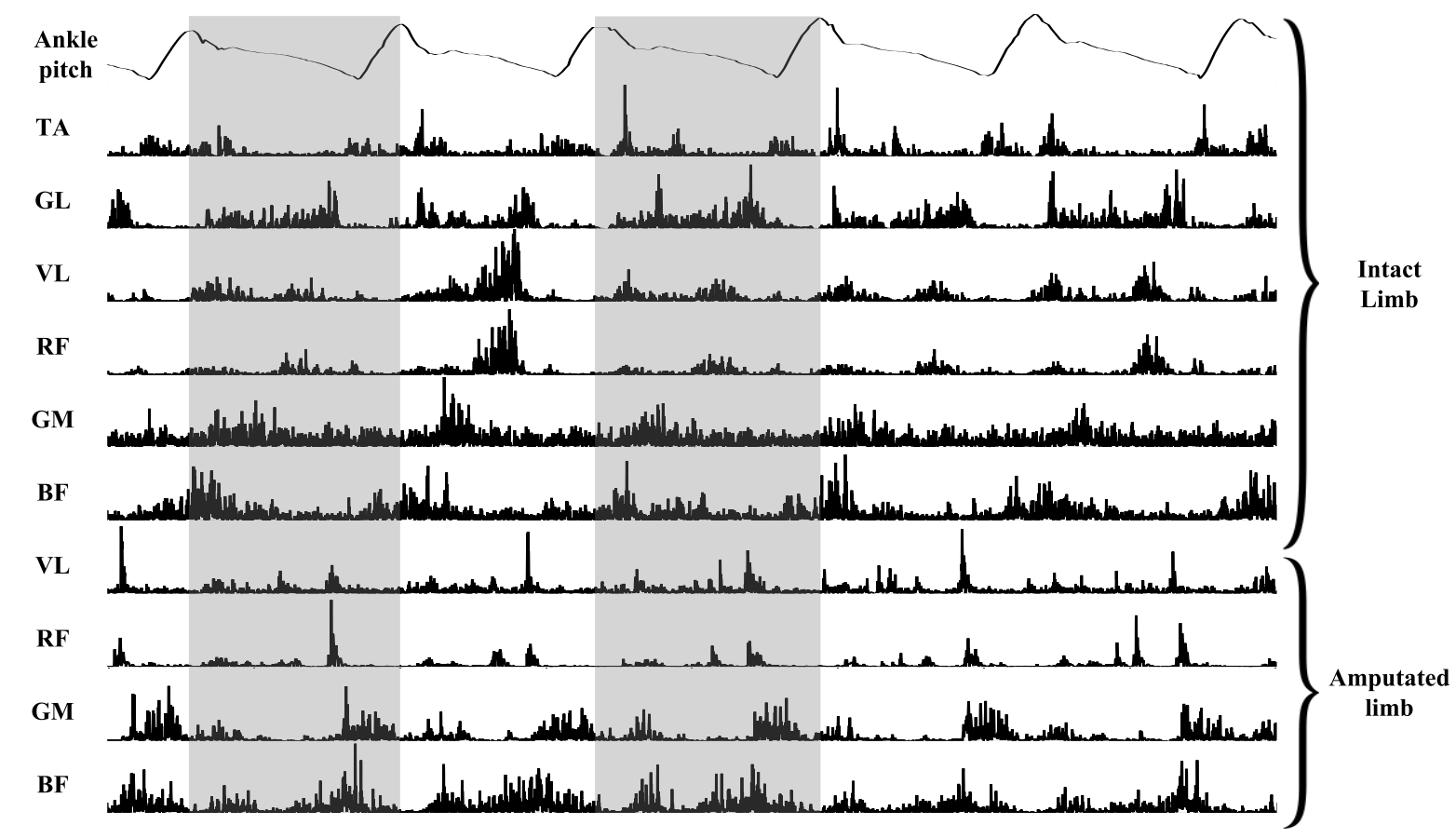

Figure 10. 10 channels of EMG from lower limb of subject 1 while operating PPA with zero percent power and zero percent stiffness for five steps. The pitch signals depicted on top was used to detect each step and it was used to partition EMG signals to each step. Two steps detected from the pitch signals are highlighted.

As mentioned before, EMG signals are non-stationary and they need preprocessing to be able to extract information from them. Figure 10, shows 10 channels of synchronized filtered and rectified EMG and the pitch signal with detected extremums for step partitioning. To quantify the EMG signals, simple feature extraction was implemented using the amplitude of the rectified signals. The feature selection was based on the fact that the EMG spectrum depends on the firing of motor units, and it has been shown that it could be used to provide a sensitive measure for activity [92] and the amplitude of the signal shows the summation of action potentials of motor units firing around the electrode placement. It has been shown that the increase of load on muscle increases the amplitude of the motor unit action potentials and, as a result, the amplitude of EMG increases as well $[93,94]$. It was assumed that by focusing on the amplitude of the EMG signals, it is possible 
to estimate a measure to model the energy expenditure or load-bearing on the residual limbs. For this purpose, mean absolute value (MAV) feature was extracted as the following:

$$
M A V=\frac{1}{N} \sum_{i=1}^{N}|x[i]|
$$

Where $\mathrm{N}$ is the length of the EMG segment and $x$ is the EMG signal.

MAV feature is extracted from each of the ten muscles and the average of the data collected from the first recording ( 0 Stiffness and 0 power) was used to normalize the features. The normalization coefficients are calculated as follows:

$$
n_{j}=\frac{1}{M} \sum_{k=1}^{M}\left|M A V_{j}[k]\right|_{\substack{S=0 \\ P=0}}
$$

Where, $\mathrm{n}$ is the normalization factor for $j$ th muscle, $\mathrm{M}$ is the number of steps, and $\mathrm{S}$ and P represent stiffness and power parameters for PPA, respectively.

Previously, we have presented a method to use multi-channel EMG signals to model the amputee gait using a PPA, where different muscle groups were combined into a single measure using a weighted technique [81]. The weights for the muscles were calculated using fast-twitch fibre concentration [95]. Using the percentage of the fast-twitch fibres in each muscle added the physical difference of the muscles to the model. Muscle size weights are also normalized with respect to the maximum to have values between 0 to 1 and the metabolic energy estimation model is calculated as follows: 


$$
E(s, p)=\sum_{i=1}^{N} w_{i} \times \frac{M A V_{i}(s, p)}{n_{i}}
$$

Where, $w_{i}$ is the normalized weight based on the concentration of fast-twitch fibers for the $i$ th muscle and $M A V_{i}$ is the value of the phase-dependent amplitude feature for the $i$ th muscle, and $n_{i}$ is the normalization ration for $i$ th muscle.

The measure $E(s, p)$ is a function of the parameters, which is the underlying model. But, due to lack of data and non-stationary nature of EMG, it is unknown and it is calculated in a data-driven method here.

\subsubsection{Smart Search}

Utilization of equation (2-3) to identify the best parameters for each user would require doing an exhaustive search over all the possible combinations for stiffness and power parameter. As mentioned before, doing ES is accurate but unpractical due to the time constraint. In addition, due to the subject variability and non-stationary nature of the EMG signals, the underlying model for energy is unknown. In this study, a data-driven smart search optimization was proposed to automatically tune the PPA and optimize equation (23) without having to collect data for all the possible combinations. Equation (2-2) used as the objective function for stiffness and power parameters and the optimization was done as follows: 


$$
\begin{aligned}
& \underset{p, s}{\operatorname{argmin}} E(p, s) \\
& \text { s.t. } 0 \leq p \leq 50 \\
& 0 \leq s \leq 100
\end{aligned}
$$

Eq.

Where, $\mathrm{E}$ is the measure calculated using equation (3), $\mathrm{p}$ is the power parameter, and $\mathrm{s}$ represents the stiffness parameter. As mentioned in 3.2, the upper limits for parameters were determined to prevent overpowering.

The study explored more effective searching framework by employing heuristic and numerical methods to do the smart search to find the optimal tuning parameters. The data was collected offline from 121 combinations of stiffness and power where stiffness varied from 0 to 100 percent with a resolution of 10 percent and power varied from 0 to 50 percent with a resolution of 5 percent. To have a higher resolution, the collected data was interpolated to include a 1 percent change in the objective function. The final grid contains 5151 possible combinations of the parameters. To avoid doing EH over 5151 parameters, alternative optimization algorithms of Genetic Algorithm and the Nelder-Mead Simplex methods were used in this study. In addition, a Latin Hypercube Sampling approach was introduced to modify the NM method.

\subsubsection{Genetic Algorithm}

Genetic algorithm is a heuristic search method inspired by Darwin's evolution theorem. GA consists of four phases - initial population, fitness function, selection, crossover and mutation. GA starts with an initial population where each set is a possible solution, which in this case is the parameter combination. Each population is represented in the form of binary genes. Then the population is evaluated by a fitness function. In this study, the 
fitness function is equation (3). Based on the fitness value, the population is organized and in the next phase two individuals are selected for reproduction. After parent selection, in the crossover phase a crossover point in the chromosome is chosen to make offspring by exchanging the genes of parents among themselves. To maintain diversity within the population mutation is done by randomly switching the values in a gene. This process is repeated until the stopping criteria are met or maximum iterations are reached. In this study, GA was modified to have integer values for parameters and equation (3) was used as the fitness function. The algorithm was explored using various number of population to compare the speed and accuracy of the results.

\subsubsection{Nelder-Mead Simplex Method}

The Nelder-Mead method is a numerical method that is commonly used to find the minimum or maximum of a multidimensional function and it can be applied to nonlinear optimization problems where the derivatives may not be known. Its main strengths are that it requires no derivatives to be computed and that it does not require the objective function to be smooth.

The NM method attempts to minimize a scalar-valued nonlinear function of $n$ variables using only function values without any derivative information [96]. It is a direct data-driven searching method. NM uses geometrical shapes where the corners are the vertices and the fitness of each vertex is used to expand or contract to converge to the optimal point. The worst vertices where $E(p, s)$ has the biggest values is replaced with new vertices in each iteration and a new triangle is formed. The vertices are updated in each iteration to form a new shape where the value of the function gets smaller and smaller until the optimal point 
is found. In this study, various number of vertices was investigated to find the smallest number of vertices need for this problem. The NM method iteratively generates a sequence of vertices to approximate an optimal point [97]. The method starts by randomly generating $n+1$ vertices [98]. At each iteration, the vertices are ordered according to the cost function values. In this study, cost function defined in equation (3) was used to find power and stiffness values. Considering equation (3), the ordered cost functions will be calculated as follows:

$$
E\left(p_{1}, s_{1}\right) \leq E\left(p_{2}, s_{2}\right) \leq \cdots \leq E\left(p_{n+1}, s_{n+1}\right)
$$

Where, $E$ denotes the cost function, and $p_{i}$ and $s_{i}$ are randomly generated values for stiffness and power parameters. Since we are dealing with physical parameters, the random generation for the values was limited to suit the current problem. Power parameter was limited to 0 to 50 percent and stiffness limited to 0 to 100 percent. In addition, randomly generated values are limited to integer values as the resolution of the system cannot go under 1 percent. The algorithm uses four possible operations - reflection, expansion, contraction, and shrink - each being associated with a scalar parameter. Based on the value of the cost function, these possible operations are used to calculate the new point. After initial $n+1$ vertices are organized from best to worst, the centroid for the $n$ best vertices are calculated as follows: 


$$
\bar{c}=\frac{1}{n} \sum_{i=1}^{n} c_{i}
$$

Where, $c_{i}$ is a vector of the parameters as $\left[p_{i}, c_{i}\right]$ in the $i$ th vertices, $\mathrm{n}$ is the number of vertices, and $\bar{c}$ is the centroid which shows the coordinate of the centroid parameters. Using the centroid, a reflection point can be calculated as follows:

$$
c_{r}=\text { floor }\left(\left\lfloor\bar{c}+\alpha\left(\bar{c}-c_{n+1}\right)\right\rfloor\right)
$$

Where, $\alpha$ is the reflection rate and the value is floored to have an integer value to fit the current problem as the parameters have the constraint to obtain integer values. Following that, the fuction gets evaluated in the reflection point and if $E_{1} \leq E_{r} \leq E_{n}$, then the $n+1$ vertices are replaced by $c_{r}$.

However, if $E_{r}<E_{1}$, then the expansion point needs to be calculated to cover the area with a smaller value that was missing in the previous iteration. The expansion point is calculated as follows:

$$
c_{e}=\operatorname{floor}\left(\left\lfloor\bar{c}+\beta\left(c_{r}-\bar{c}\right)\right\rfloor\right)
$$

Where, $\beta$ is the expansion rate. The expansion point is evaluated and if $E_{e}<E_{r}, c_{n+1}$ is replaced by $c_{e}$. Otherwise, $c_{n+1}$ is replaced with $c_{r}$.

If the reflection point is $E_{n} \leq E_{r}<E_{n+1}$, then the algorithm needs to compute a contraction point outside and evaluate it as follows:

$$
c_{c}=\text { floor }\left(\left\lfloor\bar{c}+\gamma\left(c_{r}-\bar{c}\right)\right\rfloor\right)
$$

Where, $\gamma$ is the contraction rate. If $E_{c} \leq E_{r}$, then contraction point replaces the $n+1$ point and, otherwise, the algorithm shrinks the data. Before going to the shrinking part, we 
need to consider the case where reflection point is bigger than $n+1$ point $\left(E_{r} \geq E_{n+1}\right)$. In this case, we also need to contract the data inside using equation (6). Evaluating the current contraction point, if it is smaller than $n+1$ point, it replaces that. Otherwise, the data needs to shrink as follows:

$$
c_{i}=\text { floor }\left(\left\lfloor c_{1}+\delta\left(c_{i}-c_{1}\right)\right\rfloor\right)
$$

Where, $\delta$ is the shrink rate. Conventionally, the reflection, expansion, contraction and shrink rates are user-defined, but in this study, we used the adaptive method presented by Gao et al. to implement the ND [97]. The adaptive parameters for NM method are calculated as the following:

$$
\alpha=1, \quad \beta=1+\frac{2}{n}, \quad \gamma=0.75-\frac{1}{2 \times n}, \quad \delta=1-\frac{1}{n}
$$

The method was modified to fit our problem by limiting the parameters to integer values, which represent the 1 percent resolution and the new point in each iteration was limited to lower and upper bands of the parameters. The algorithm also evaluated using various numbers of vertices to explore the smallest number required for tuning. The dimension of the vertices (n) was varied from 5 to 20 to evaluate the convergence accuracy and number of necessary iterations.

\subsubsection{Modified Nelder-Mean Complex with Latin Hypercube Sampling}

If there are multiple local minima with small differences in the optimization problem the NM might fail to converge to the global minima and is stuck in local minima. In addition, searching through a greater area might take more iterations until the target 
optimization value is achieved. To resolve this issue and improve the results, it is hypothesized that using a sampling method would improve the searching accuracy and find the minima in fewer iterations. For numerical problems, the Monte Carlo methods are usually more efficient to do sampling from high dimensional probability distributions [99]. However, a great number of samples are typically required in the traditional Monte Carlo method to achieve good accuracy. Monte Carlo, which is a random sampling method, is the easiest method for sampling, but there is no assurance that a sample element will be generated from any particular subset of the sample space $[100,101]$. There are techniques to improve accuracy by controlling the sample points. Latin Hypercube Sampling is a widely used method to generate controlled random samples [102]. The basic idea of LHS is to divide probability distributions into intervals of equal probabilities and a sample is taken out of each interval [103-105]. In this study, two-dimensional LHS was used to obtain sample points to determine the best search area. The two-dimension LHS was used with the assumption that power and stiffness parameters are independent, their probability distributions are evenly partitioned into $\mathrm{N}$ regions, and one sample point is randomly selected from each region. By evaluating the samples, the area for global minima is located. Using the located area, the searching method was limited to this area to keep the algorithm from being stuck in local minima or taking more iterations to find the global minima. In this study, LHS sampling was combined with the NM method to improve the smart search result and converge to the optimal setting faster. Using LHS, NM method was restricted into the area specified by the sampling technique. Various initial points were investigated to evaluate the speed and accuracy of the tuning for PPA. 


\subsection{Results}

Figure 3 shows the baseline grid for 5150 parameter combinations and the value of the estimated energy from EMG signals for six subjects (3 amputees, 3 or 4 simulated amputees). The grid of parameters shown here is collected using wireless sensors for 121 combinations of the parameters and it was interpolated to have a resolution of 1 percent (smallest change in the parameters). The amplitude of the figures is showing the estimated energy using equation (3). The subject variability is clearly observable on the grids and each subject has a different measure in the obtained grid. TF2 was not able to finish the study and only half the grid was obtained. The optimization was still implemented for the obtained half-grid for this subject.

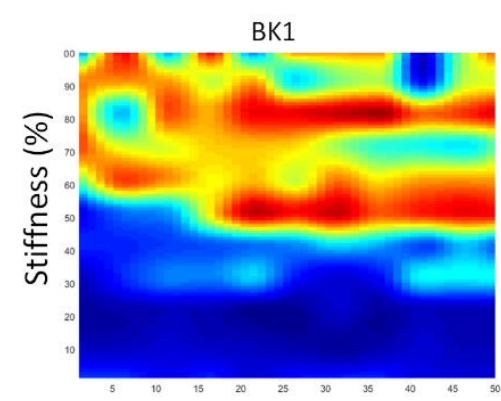

BK3

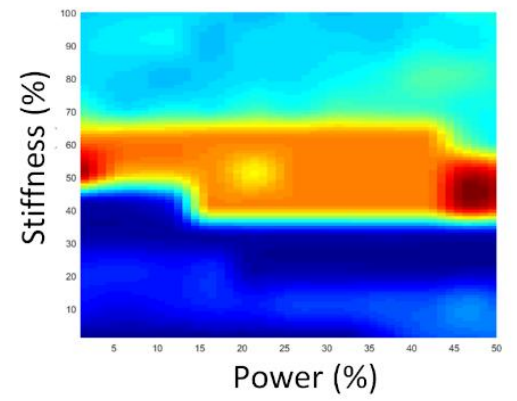

BK2

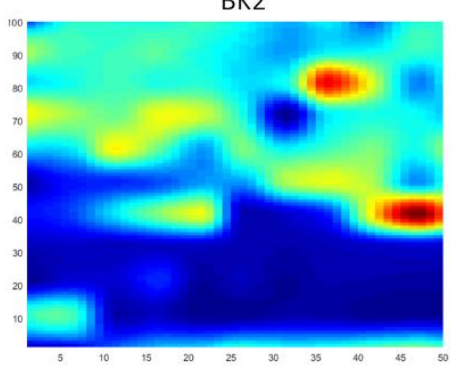

TF2

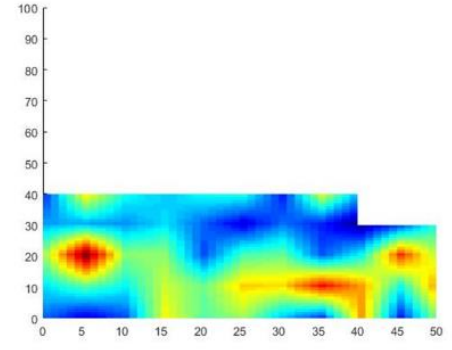

Power (\%)
TF1

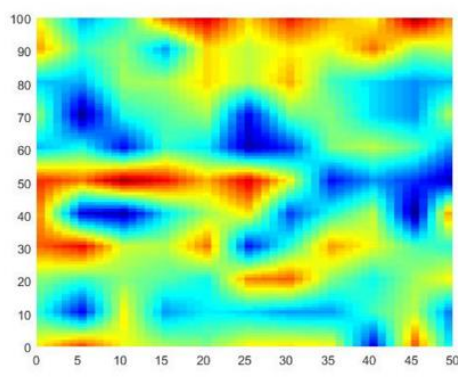

TF3

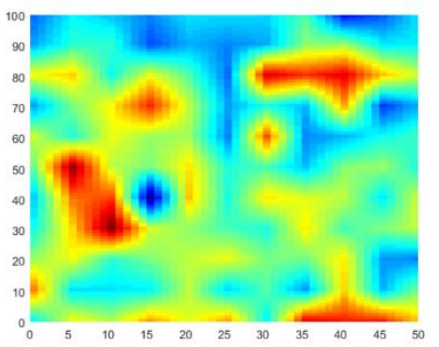

Power (\%)

Figure 11. Grids of the estimated metabolic energy model using a 10-channel EMG sensor for various combinations of the stiffness and power parameters for three bent-knee and three transfemoral amputee subjects. 
The best parameters for each user is chosen to be the minimum for the estimated energy in the grid, representative of the correct tuning value. The collection of all possible parameter combinations for 10 steps (approximately, 10 seconds) for 5150 parameter combinations would require the operation of the PPA for over fourteen hours, which is not practical and collection is not time-effective.

Table 1 shows the results of the various methods presented in this study for each subject. It could be seen that GA has the best accuracy in finding global minima. However, it requires more than 60 iterations and, in each iteration, there is a population that needs to be collected. The results are shown for a population of 15 . In addition, the investigation of NM showed it also converges to the target in much shorter time (less than 40 iterations) but the variance is higher. Addition of boundaries using LHS reduced the variance and improved the search results although requiring slightly more iterations.

Table 1. Results of the EMG-based smart search to tune PPA.

\begin{tabular}{|c|c|c|c|c|c|c|c|c|}
\hline \multirow[t]{2}{*}{ Subject } & \multirow[t]{2}{*}{$\begin{array}{c}\text { CPO } \\
\text { Tuning }\end{array}$} & \multirow[t]{2}{*}{ Target $^{1}$} & \multicolumn{2}{|c|}{ Genetic Algorithm } & \multicolumn{2}{|c|}{ Nelder-Mead } & \multicolumn{2}{|c|}{$\begin{array}{c}\text { Nelder-Mead with } \\
\text { LHS }\end{array}$} \\
\hline & & & Iterations & Result & Iterations & Result & Iterations & Result \\
\hline BK1 & $\begin{array}{l}\mathrm{P}=25 \\
\mathrm{~S}=25\end{array}$ & $\begin{array}{l}P=22 \\
S=21\end{array}$ & $66 \pm 12$ & $\begin{array}{l}P=22.3 \pm 2 \\
S=20.7 \pm 1.4\end{array}$ & $35 \pm 9$ & $\begin{array}{l}P=25 \pm 7 \\
S=17 \pm 5\end{array}$ & $49 \pm 9$ & $\begin{array}{l}P=24 \pm 3 \\
S=19 \pm 2\end{array}$ \\
\hline BK2 & $\begin{array}{l}\mathrm{P}=20 \\
\mathrm{~S}=40\end{array}$ & $\begin{array}{l}P=23 \\
S=11\end{array}$ & $60 \pm 7$ & $\begin{array}{c}P=25.8 \pm 7.2 \\
S=11.1 \pm 1\end{array}$ & $28 \pm 13$ & $\begin{array}{l}P=31 \pm 10 \\
S=14 \pm 8\end{array}$ & $45 \pm 8$ & $\begin{array}{l}P=25 \pm 6 \\
S=12 \pm 2\end{array}$ \\
\hline TF1 & $\begin{array}{l}P=40 \\
S=32\end{array}$ & $\begin{array}{l}P=45 \\
S=26\end{array}$ & $58 \pm 11$ & $\begin{array}{l}P=44 \pm 3 \\
S=25 \pm 3\end{array}$ & $21 \pm 8$ & $\begin{array}{l}P=28 \pm 15 \\
S=23 \pm 9\end{array}$ & $57 \pm 15$ & $\begin{array}{l}P=43 \pm 3 \\
S=25 \pm 1\end{array}$ \\
\hline BK3 & $\begin{array}{l}P=40 \\
S=65\end{array}$ & $\begin{array}{l}P=46 \\
S=41\end{array}$ & $63 \pm 10$ & $\begin{array}{l}P=40 \pm 12 \\
S=40 \pm 6\end{array}$ & $10 \pm 4$ & $\begin{array}{l}P=27 \pm 15 \\
S=51 \pm 18\end{array}$ & $24 \pm 13$ & $\begin{array}{c}P=26 \pm 0.7 \\
S=62 \pm 1\end{array}$ \\
\hline TF2 & $\mathrm{P}=40$ & $\mathrm{P}=41$ & $58 \pm 4$ & $\mathrm{P}=39 \pm 8$ & $11 \pm 8$ & $\mathrm{P}=26 \pm 12$ & $13 \pm 8$ & $\mathrm{P}=40 \pm 1$ \\
\hline
\end{tabular}




\begin{tabular}{|c|c|c|c|c|c|c|c|c|}
\hline & $\mathrm{S}=40$ & $\mathrm{~S}=31$ & & $\mathrm{~S}=29 \pm 7$ & & $S=25 \pm 11$ & & $S=31 \pm 1$ \\
\hline TF3 & $\begin{array}{l}P=23 \\
S=36\end{array}$ & $\begin{array}{l}P=16 \\
S=41\end{array}$ & $62 \pm 10$ & $\begin{array}{l}\mathrm{P}=28 \pm 15 \\
\mathrm{~S}=31 \pm 11\end{array}$ & $9 \pm 4$ & $\begin{array}{l}\mathrm{P}=29 \pm 19 \\
\mathrm{~S}=33 \pm 14\end{array}$ & \pm & $\begin{array}{r}\mathrm{P}=16 \pm 0.6 \\
\mathrm{~S}=92 \pm 2\end{array}$ \\
\hline
\end{tabular}

$1 \mathrm{P}$ and $\mathrm{S}$ represent Power and Stiffness parameters for PPA respectively. BK stands for bent-knee and TF

for transfemoral.

The GA is implemented to do the search over the combinations in a heuristic way to have a smarter alternative to the ES. Population sizes from 5 to 30 were investigated to explore the practicality of the search. Figure 12 shows the error bars for GA with various population sizes for each subject. The error is calculated as the Mahalanobis distance from the target point. With the increment in the population size, the error reduces, and the algorithm has a better convergence to the global minima. However, GA is population-based and every iteration requires updated population and collection of data for all the members of the population. Error! Reference source not found. shows the results of a search for a 11 the methods and the number of iterations before convergence. 


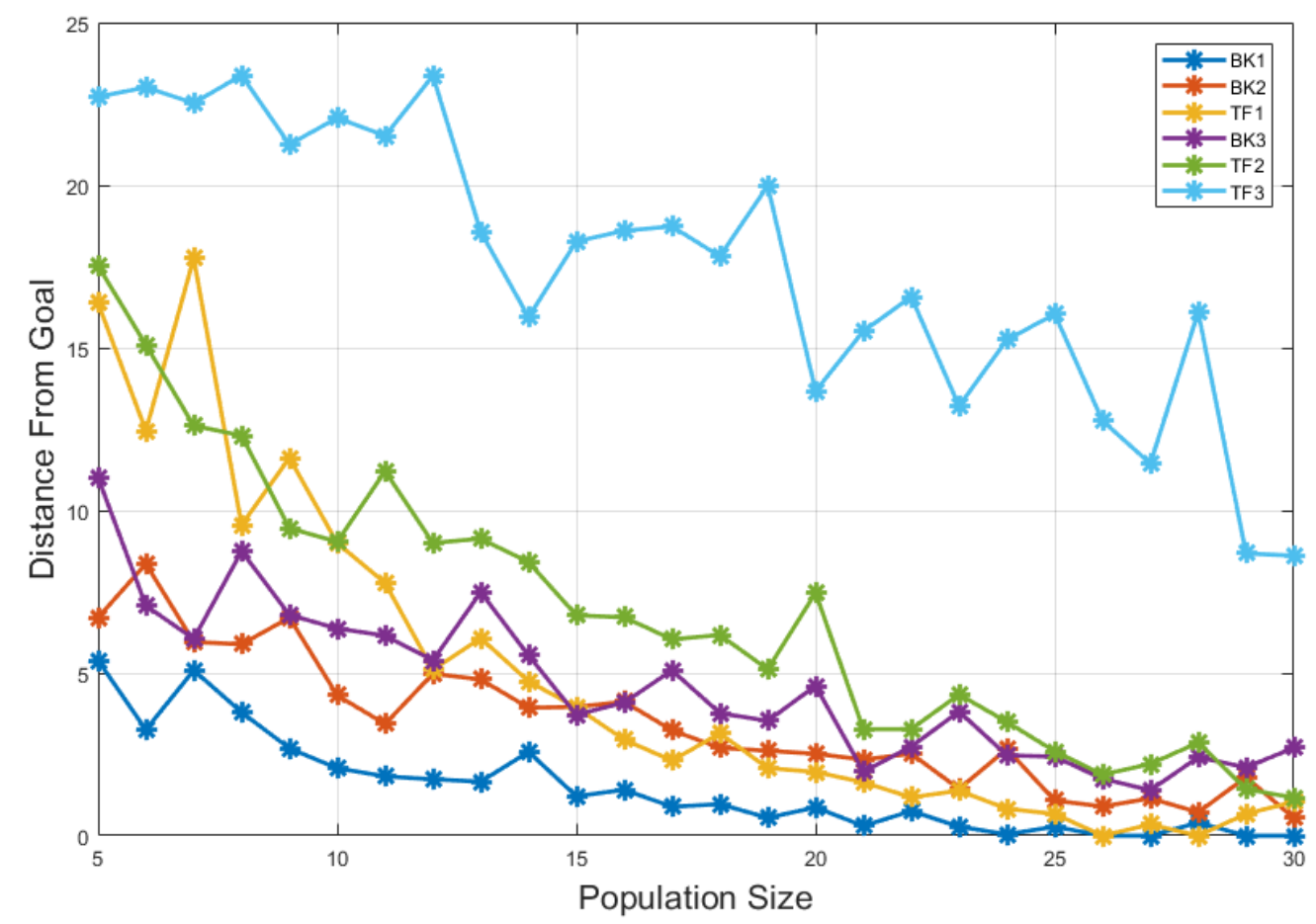

Figure 12. Error for smart search using the GA method in various population sizes. GA method was investigated using various initial population sizes to identify the smallest population size needed to converge to the global minima faster. The results are evaluated using an error measure which is the distance of the result of optimization from the target value.

The NM method was explored using different vertices by sweeping $\mathrm{n}$ from 3 to 100 . Since the initialization is random, each $\mathrm{n}$ was repeated 1000 times and the mean error was calculated. Figure 13 (a) shows the mean error for each vertex (n). The smallest number of $\mathrm{n}$ was chosen to be 10. Later, the NM method was explored by applying the search boundary determined by LHS. LHS was explored with various starting points and the starting points are swept from 4 to 30 . Figure 13 (b) shows the search results using various sampling points to bound NM. The best number of initial sampling was chosen to be 5 . 

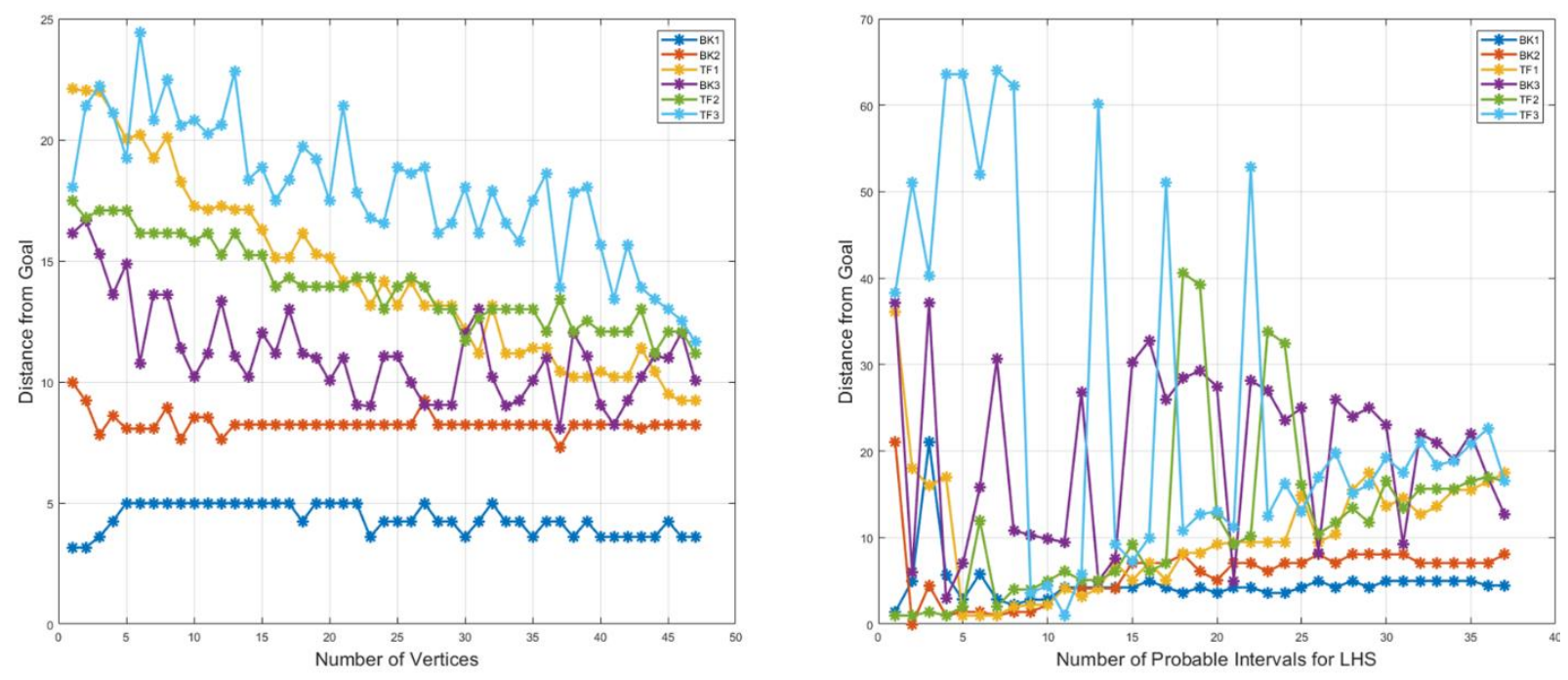

Figure 13. Error for smart search using NM and LHS methods in various vertices and random samples. (a) The distance from the target value for various simplexes. (b) The distance for the NM optimization using LHS with a various number of intervals to limit the search.

\subsection{Discussion}

In this study, a smart data-driven optimization method was used for automatic PPA tuning. A wireless EMG sensor system was used to collect physiological data from the residual limbs of the subjects. Previously, some studies focused on automatically tuning prosthetic knees and ankles using mechanical sensors and did not consider the physiological aspect of the gait. The current study hypothesized that the use of physiological data could result in accurate tuning. The reason for this hypothesis was based on the fact that the mechanical sensors collect information after the physical movement was made, while it is possible to use physiological data and observe the effect earlier. Also, physiological data could be linked to the energy expenditure which can be used to optimized the performance of PPA. For physiological sensors, EMG signals were collected 
from the residual limbs. EMG signals are analyzed and combined using a weighted technique where the weights reflected the size variability of the muscles based on the percentage of fast-twitch fibres.

The physiological data were processed and used to model estimation for energy expenditure. The estimated energy model was investigated to optimize the performance of PPA by minimizing the energy where the parameters were manipulated to tune the PPA. It was hypothesized that normal gait has optimal energy expenditure and the PPA should provide the smallest energy burden on the subject to have the best performance. The current methods of the tuning for PPA are solely based on the observation of the CPO and there is no tool to quantify the fitness of the leg. By introducing an EMG-based model, we presented an evidence-based alternative. The model considers the size difference of the various muscle groups by assigning a weight to the normalized amplitude feature of each muscle. It has been shown before that a similar method can be used to tune a PPA [81].

However, the best way to tune using the EMG-based method is to investigate the performance on all the possible combinations for stiffness and power parameters. Considering ten steps for each parameter combination, which is approximately ten seconds, would lead to a tuning session that would span over 14 hours which does not provide the time-efficiency for practicality. The focus of this study was to investigate the possibility of using adaptive data-driven optimization methods to achieve parameter selection and avoid ES. GA and NM approaches were implemented and, later, a modification was added to NM method using LHS to improve the search. GA resulted in the closest results to the target and smallest variance in the answers in comparison to others. GA was repeated 100 times; the results are reported for the average of the repetitions and it is worth noting that 
over 90 percent of the results were exactly the target value. However, as expected, since this approach is population-based and requires having multiple combinations in every iteration, it requires many cases to be considered and, even though it improved the search with comparison to ES, it still is impractical for tuning application. In this study, the NM simplex method was also proposed, where a geometrical simplex is manipulated by manipulating the shape using the fitness of the corners. A simplex, depending on the number of corners, would be a triangle, foursquare and so on. We investigated a triangular simplex up to 100 simplex shapes to identify the smallest simplex to optimize the problem. Figure 5 showed the change in the mean difference from the target for 100 repetitions and 10 simplexes were chosen as the best simplex for this purpose. The results of NM method showed an average of fewer than 40 iterations to set up parameters, which indicates the possibility of doing automatic tuning in approximately 6 minutes on average. However, the variance of the results was more than 5 percent, which increases the inconsistency of the approach and might lead to overpowering or under-powering of the PPA and miscalculation for stiffness. For example, the variance for power of TF1 was 15 , which is very large and would not be practical to have this much inaccuracy. The small change in the estimated energy, which can be seen in Figure 3, is the reason for the big variance. Introduction of LHS to NM method helped limit the search to the area where global minima were located, improved the results, and reduced the variance. However, the modified version converged to target values in more iterations. It took 75 iterations at most, which is approximately 12 minutes, for modified NM to tune the leg. The modified NM method using LHS resulted in better accuracy in comparison to NM but it took longer to tune. However, the longer tuning time for modified version was still short enough to be practical 
and it can be used to replace the conventional subjective methods. In comparison to GA, modified NM was not as accurate but it required significantly less time.

The results of the study showed the feasibility of using physiological data to objectively tune a PPA automatically and accurately. In addition, the lack of a universal model for amputee gait due to the subject variability of EMG signals was resolved by relying on the data itself. The NM collected data in a geometrical sense and, based on the fitness, the geometrical search area expanded or contracted to converge to the global minima, which are the best calibration values for PPA parameters. Future work will focus on implementing a real-time system to be tested outside of a controlled environment.

\subsection{Conclusions}

This study demonstrated the feasibility of using EMG signals from the residual limbs of amputees to model estimation for gait energy expenditure. In addition, a modified NM optimization method was presented and its results show that it is possible to use the NM method as a smart automatic calibration tool for the setup of the PPA parameters. PPA calibration can be done objectively using the presented method based on physiological evidence in a time-effective way. 


\section{CHAPTER 3 ADAPTIVE CONTROL OF POWERED PROSTHESES}

\subsection{Introduction}

The advent of powered prosthetic ankle systems revolutionized the assistance for amputee gait. The current technology provides net-positive power in the toe-off to facilitate the completion of steps. Furthermore, the PPA systems embedded with spring-based joints and motors that introduce resistance to gait by imitating the ankle movement and the muscles increasing balance. PPA systems have shown to reduce the metabolic energy expenditure and have been very effective in restoring balance and symmetry in gait [19, 30]. In addition, PPA systems are enhanced with impedance-controlled parameter such as the power and stiffness of the joint that makes them suitable for users with different physical features. The adjustable parameters also allow the system to provide various amounts of assistance for different terrain types and intentions. Even though PPA systems are capable of being adaptive, but current systems are lacking the required adaptivity on uneven terrains to provide optimal assistance.

Thus, it is crucial to be able to understand the underlying terrain and provide adaptive assistance to the user. In this study, a wide range of sensors such as Electromyogram (EMG), Inertial Measurement Units (IMU), Infrared Sensor (IR), Piezo-Resistive Pressure Sensors (PS) used to predict the changes in the locomotion mode before transitioning to the new terrain and adapt the system before heel contact (HC) of the prostheses. A novel real-time sensor-fusion method developed for prediction of the locomotion mode a step ahead of the time using machine-learning techniques to enhance the prosthetic control system and improve gait. 


\subsection{Related works}

Conventionally passive prostheses systems are used to replace lost limbs due to their relative simplicity, low-cost and robust design that helps bring back partial function restoration. The inherent shortcomings of these devices are their inability to generate mechanical power, lack of adaptivity to intention and, lack of sensory feedback [106]. Recent developments in the prosthesis field introduced powered prosthetic systems that are capable of providing net-positive power and it has been shown that they improve the gait while reducing the metabolic energy expenditure [30, 107, 108].

While such technologies have proven to be highly effective, additional challenges remain regarding the control system of such technologies. Such as how early the transition step should be detected and how the system should respond to a change in the intention are still required to be investigated. There have been studies focusing on detecting the terrain type to prototype adaptability. Several studies have focused on detection of the intention after the step happens and the challenge of predicting the intention is still requires more attention. Jin et al. [109] focused on electromyographic signals and extracted various features from healthy subjects and used a threshold-based method to detect the terrains in the lab environment. Huang et al. $[110,111]$ also used EMG signals to classify locomotion modes and used foot switch and motion systems to obtained the gait phases in the lab and showed the possibility of detecting various intentions. Varol et al. [112] used joint angles and angular velocities for intent recognition and showed it is feasible on one subject. Hargrove et al. [113] used the nerves from the amputated limb and did an invasive reinnervation surgery to transfer the nerves on residual thigh muscles and used these muscles to control a prosthetic leg. Wentik et al. [114] used EMG and kinetic data to detect the 
initiation of the gait and their results showed it could detect the toe-off and heel-contact in non-amputees. Hoover et al. [115] used EMG signals to detect the stair descent using EMG, and kinematic sensors. Spanias et al. [116, 117] combined various sensors and showed that it could reduce the error in intention detection. Review of the related works are presented in Table 2

Table 2. Review of the related works for PPA control

\begin{tabular}{|c|c|c|c|c|}
\hline Year & Author & Intentions & Accuracy & Description \\
\hline 2006 & Jin [109] & Slope, Stair, Level & $75>$ & $\begin{array}{l}\text { EMG in lab setting, threshold- } \\
\text { based classification }\end{array}$ \\
\hline 2010 & Varol [112] & Stand, Sit, Walk & 100 & $\begin{array}{l}\text { Joint angles and angular velocities, } \\
\text { One transfemoral subject }\end{array}$ \\
\hline 2011 & Huang [110] & $\begin{array}{l}\text { Slope, Stair, Level, } \\
\text { Obstacle }\end{array}$ & 95 & EMG, Ground reaction forces \\
\hline 2012 & Wentik [114] & Gait initiation & - & $\begin{array}{l}\text { EMG and inertial data from non- } \\
\text { amputees }\end{array}$ \\
\hline 2013 & Hargrove[113] & Level, Stair, Ramp & $86.8>$ & $\begin{array}{l}\text { EMG from surgically reinnervated } \\
\text { residual thigh muscles }\end{array}$ \\
\hline 2018 & Spanias [116] & Slope, Stair, Level & $88.46>$ & $\begin{array}{l}\text { EMG, Kinetic, Kinematic } \\
\text { information over multiple days }\end{array}$ \\
\hline
\end{tabular}

However, these studies mostly focused on detecting the terrain after the users shifted from one terrain to another. To be more specific the terrain or detection was done after the $\mathrm{HC}$ of the prosthetic leg, which would not allow time for the control system to adapt to the change. In addition, previous studies did not consider situations that might look similar to change but would not lead to change. For example, walking towards an obstacle or turning that might excite similar activity from the sensors on residual limbs that would result in false detection and increase the risk of losing balance due to false positive detection. 
An instance of how subjects might need different assistance level could be transitioning from level ground to stair ascent. In this case, subjects will require more net-positive power to help with stair terrain and failure of predicting and adapting accordingly would result in the expenditure of higher energy and less smooth transition. In order to be able to provide a real-time adaptive system, it is crucial to predict the intention before the prosthetic system is engaged in the new terrain. Likewise, for descending stairs, the system requires to reduce the power and increase the stiffness to help amputees. Figure 14 shows the various terrain types that might occur and investigated in this study. Hence, this study focused on predicting the change in terrain before the $\mathrm{HC}$ for prosthetic foot and adapting parameters to fit the new terrain. This will ensure smooth transition and optimal gait pattern.

(a)

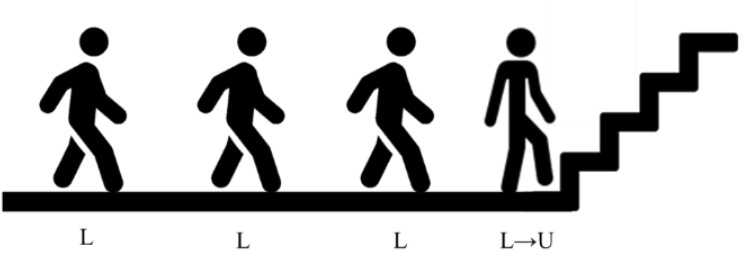

(b)

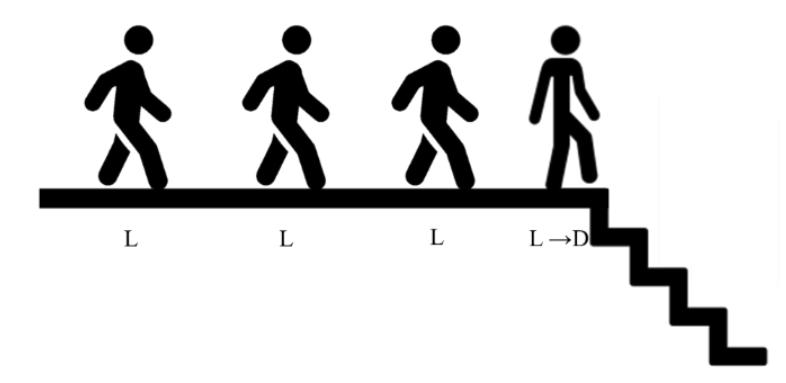

(c)

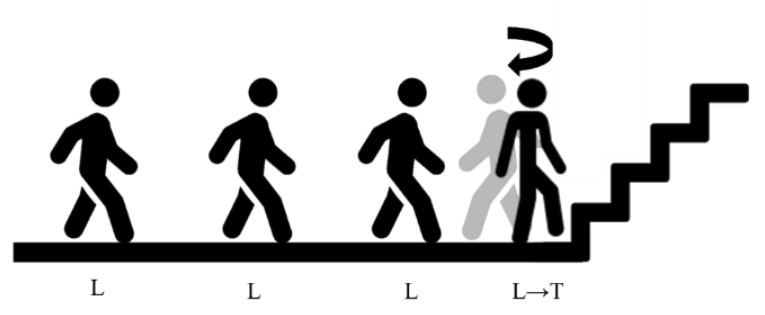

Figure 14. Transition to different terrain while using PPA. (a) The subject takes three steps on level ground and transitions to stair ascend leading with intact limb. (b) Subject walks on level ground and transitions to stair descend leading with the intact limb. (c) Subject walks toward the stairs but instead of ascending, the stairs turn and comes back. 
There are two major challenges that require investigation to achieve an adaptive control system. First, the control system requires detecting the change before it happens. Level walking is the baseline terrain that system works with and it is necessary to differentiate transition to different terrains. The first question to answer is whether the system can detect change in the locomotion mode from level ground. Following the change detection, the control system requires to predict the new terrain mode and adapt the PPA system. As different terrains require a different type of assistance, it is crucial to have accurate prediction in this situation to avoid loss of balance and possible injury. Thus, the second challenge is that whether the control system can accurately predict different terrain types.

In this study, it was hypothesized that using a combination of physiological, kinetic and kinematic information and fusing them, it will be possible to predict changes in the terrain before the transition step. In addition to the prediction of change in the terrain, the adaptive control system would be capable of differentiating between various terrain types and respond accordingly. Thus, predicting the transition to a different terrain will ensure a smooth gait with optimal assistance. According to the analysis of clinical need, this study aims to develop effective methods to support accurate prediction of user's intention to move up and down stairs before the move is made on the prosthetic leg for adaptive control of prosthesis.

In this study, a machine learning-based approach obtained to predict deviation from level ground and to predict the terrain type using the information buffered before the heel contact of the prosthetic foot. Electromyogram signals from lower leg muscles on both amputee and intact limb of transtibial below-knee amputees used as well as accelerometer 
and gyroscope data on the ankle, knee and hip from both prosthetic and intact sides for real-time prediction of following gait cycle.

The rest of the chapter is organized as follows. In section 3.3 material and methods used in this study are explained. Section 3.4 illustrates the results of the pseudo-real-time implementation of the proposed method. And in sections 3.5 and 3.6 presents the discussion of the results and conclusion, respectively.

\subsection{Material and Methods}

\subsubsection{Experimental protocol}

Two transtibial amputees (below the knee) and two simulated subjects recruited for the study. The non-amputee subjects operated the prostheses in a bent-knee configuration to simulate the amputee gait. Physical features of the subjects shown in Table 3.

Table 3. Physical features of the prosthetic control subjects.

\begin{tabular}{l|lllcll}
\multicolumn{2}{l}{ Subject } & Gender & Age & Weight $(\mathrm{kg})$ & Height $(\mathrm{cm})$ & Amputation type \\
\hline 1 & $\mathrm{M}$ & 30 & 75 & 172 & Simulated \\
2 & $\mathrm{M}$ & 27 & 69 & 173 & Simulated \\
3 & $\mathrm{M}$ & 47 & 81 & 173 & Transtibial \\
4 & $\mathrm{M}$ & 53 & 115 & 170 & Transtibial
\end{tabular}

The bent-knee simulation achieved using a custom-built socket to fit simulated subjects.

Figure 15 shows the amputee and simulate prosthetic configurations. Prior to data collection, subjects signed informed consent and the study approved by Institutional Review Board (IRB) of Florida International University and Hunter Holmes McGuire Veteran Affairs Medical Center. 


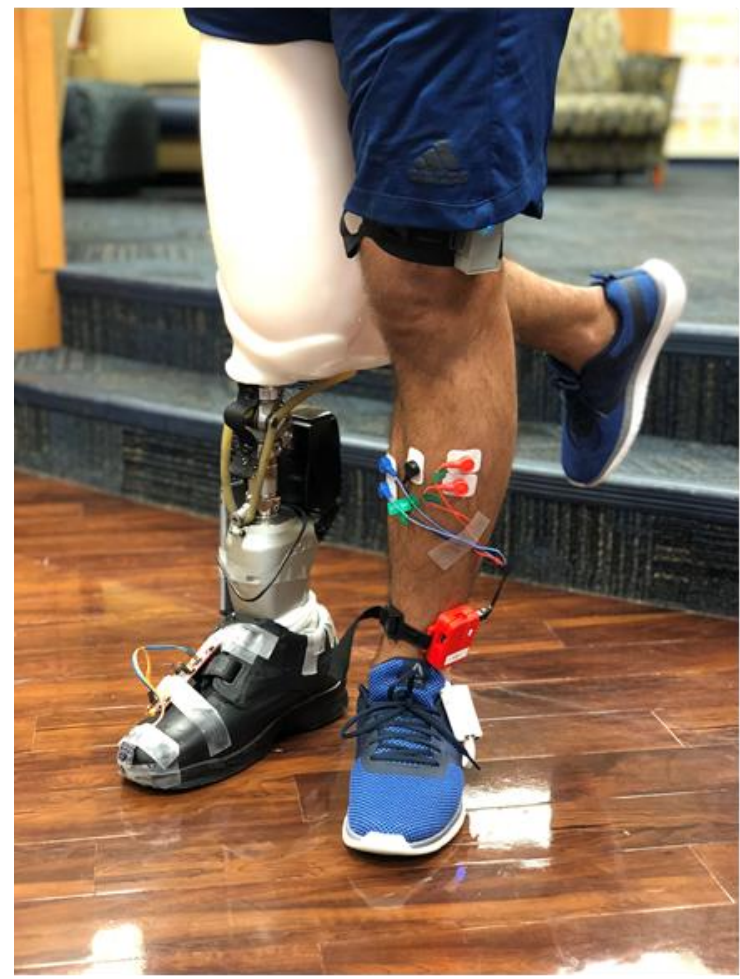

(a)

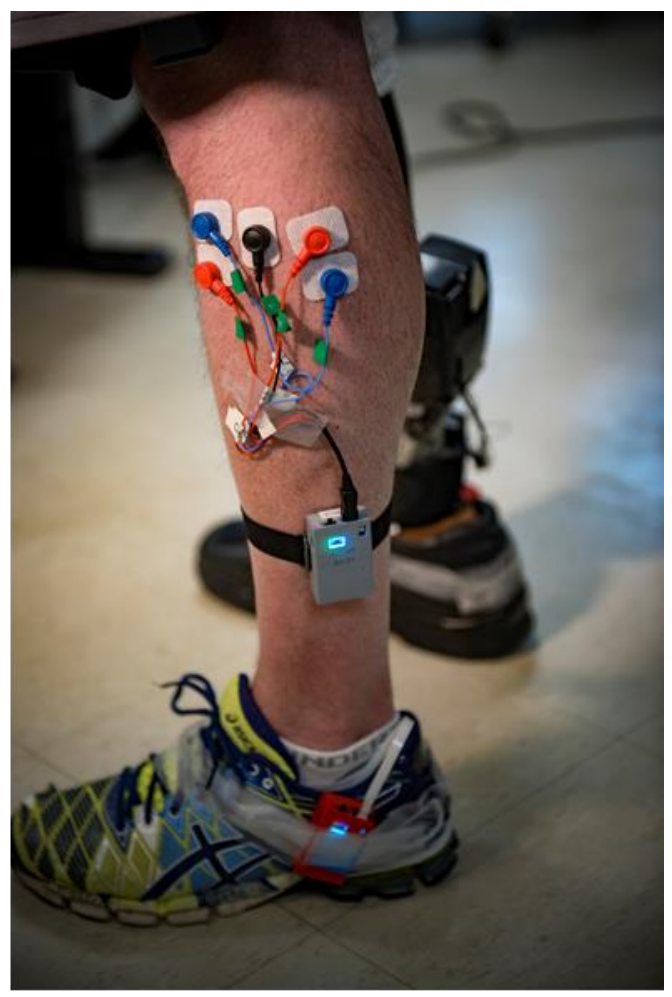

(b)

Figure 15. Simulated prosthetic user (a), Amputee subject (b)

Physiological, kinetic and kinematic data collected from subjects while operating a powered prosthetic ankle in level terrain, stairs, and turning. EMG signals collected from both intact and amputee limbs. EMG signals collected using a custom-built wireless sensor. The muscles targeted in this study are Soleus (Sol), Tibialis Anterior (TA), Rectus Femoris (RF), Vastus Lateralis (VL), Biceps Femoris (BF), Gluteus Medius (GM). Sol and TA muscles are below knee muscle and their EMG signals recorded solely from the intact side. The EMG signals sampled at $1000 \mathrm{~Hz}$ sampling frequency using $\mathrm{Ag} / \mathrm{AgCl}$ surface electrodes. Furthermore, five Inertial Measurement Units (IMU) placed on the residual limbs. IMU consists of three degree-of-freedom (DOF) accelerometer and three DOF 
gyroscope. IMU collected using custom-built wireless sensors with 1000 sampling rate and they were placed on both intact and prosthetic ankle, knees and the hips of the subjects.

For the purpose of gait phase detection, a wearable wireless ground reaction force (GRF) system designed. The GFR system consists of an insole system imbedded with three piezoresistive pressure sensors located at forefoot, mid-foot and the heel. The GRF system was placed inside the prosthetic shoe and signals recorded with $125 \mathrm{~Hz}$ sampling frequency. Finally, to be able to have an independent observation of the environment an infrared (IR) distance sensor developed and wirelessly synchronized with other sensors. The IR sensor placed on the tip of the prosthetic foot to measure the distance from the obstacles in front of it. All sensors synchronized, and data collected from subjects during gait in various terrains.

Data collection protocol consisted of level ground, stair ascent, stair descent, and turning. Subject prosthetic fitting and sensor setup was done by experts. Experts place electrodes for each muscle according to SENIAM [118] and, sensors are placed and connected. Subjects operated the PPA in three different paths. For stair ascent, subjects started walking a level terrain where they took four steps and walked up two stairs. Stair descent recording consisted of two steps in level ground followed by going down two stairs. In addition, for turning the subjects walked on level terrain for four steps toward the stairs, turned around, and walked back. Each recording repeated at least thirty times. Figure 14, shows the investigated terrain types.

In this study, BiOM ankle prosthetic system used. BiOM is the only commercially available PPA system that provides propulsion to amputees and mimics the natural gait [119]. This system models the muscle function during the stance phase by introducing the 
impedance-controlled stiffness parameter. The control system for this prosthetic is designed to provide the propulsion at the end of the load-bearing phase (stance phase) and specifically in the heel off $(\mathrm{HO})$ of the prosthetic. The amount of assistance provided during the propulsion controlled by power and stiffness parameters. The parameters calibrated for each user allowing the system to be compatible with users' physical features. The parameters are set to a fixed value and the control system fails to acknowledge the changes in the terrains and environment. The physiological and mechanical data recorded from subjects of the study to investigate fusing information for predicting the change in terrain and the type of intention.

\subsubsection{Gait phase detection}

To ensure a timely prediction of intention it is required to detect the change in the terrain before the prosthetic engagement. The ability to predict the intention will allow the system to have a smooth transition that will improve the gait performance and reduce the possibility of losing balance and falling.

Gait is a bipedal process that includes a stance and swing phases for each side. This study focuses on the prosthetic ankle and applies a phase detection algorithm to detect the start of stance phase from the GRF system. The presented algorithm detects HC in realtime. The HC detection algorithm starts with buffering the last 200ms of GRF signal. The GRF data calculated using the three pressure sensors inside the sole as following:

$$
G R F[n]=\sum_{i=1}^{3} P_{m}[n]
$$

Eq. 3-1

Where, $P_{i}$ is the pressure signal on the $i^{\text {th }}$ location inside the sole and $\mathrm{n}$ is the current time. 
The algorithm uses an adaptive threshold. The threshold calculated as following:

$$
\theta=\left\{\begin{array}{lr}
0.01 & \mu_{G R F}>0.01 \\
0.1 \times \mu_{G R F} & \text { otherwise }
\end{array}, \mu_{G R F}=\frac{1}{N} \sum_{n=1}^{N} G R F[n]\right.
$$

Where, $\theta$ is the adaptive threshold, $\mu_{G R F}$ is the average of GRF signal and $N$ is the length of the GRF signal. The value of 0.01 chosen empirically.

The GRF applied to the threshold and first-order discrete differential at time $n$ is calculated as follows:

$$
\begin{aligned}
& G R F_{C}[n]=\left\{\begin{array}{lr}
0 & \text { if } G R F[n]<\theta \\
G R F[n] & \text { otherwise }
\end{array}\right. \\
& G R F_{\text {diff }}[n]=G R F_{c}[n]-G R F_{c}[n-1]
\end{aligned}
$$

From the transformed signal, the $\mathrm{HC}$ is detected at the time $T_{H C}$ to have the following criteria:

1. $G R F_{\text {diff }}\left[T_{H C}\right]>0$

2. $\sum_{n=T_{H C^{-5}}}^{T_{H C^{-1}}} G R F_{\text {diff }}\left[T_{H C}-n\right]=0$

Figure 16 shows the GRF signal and detected HCs using the presented algorithm for a sample signal. The results of HC detection were validated by visual inspection. 


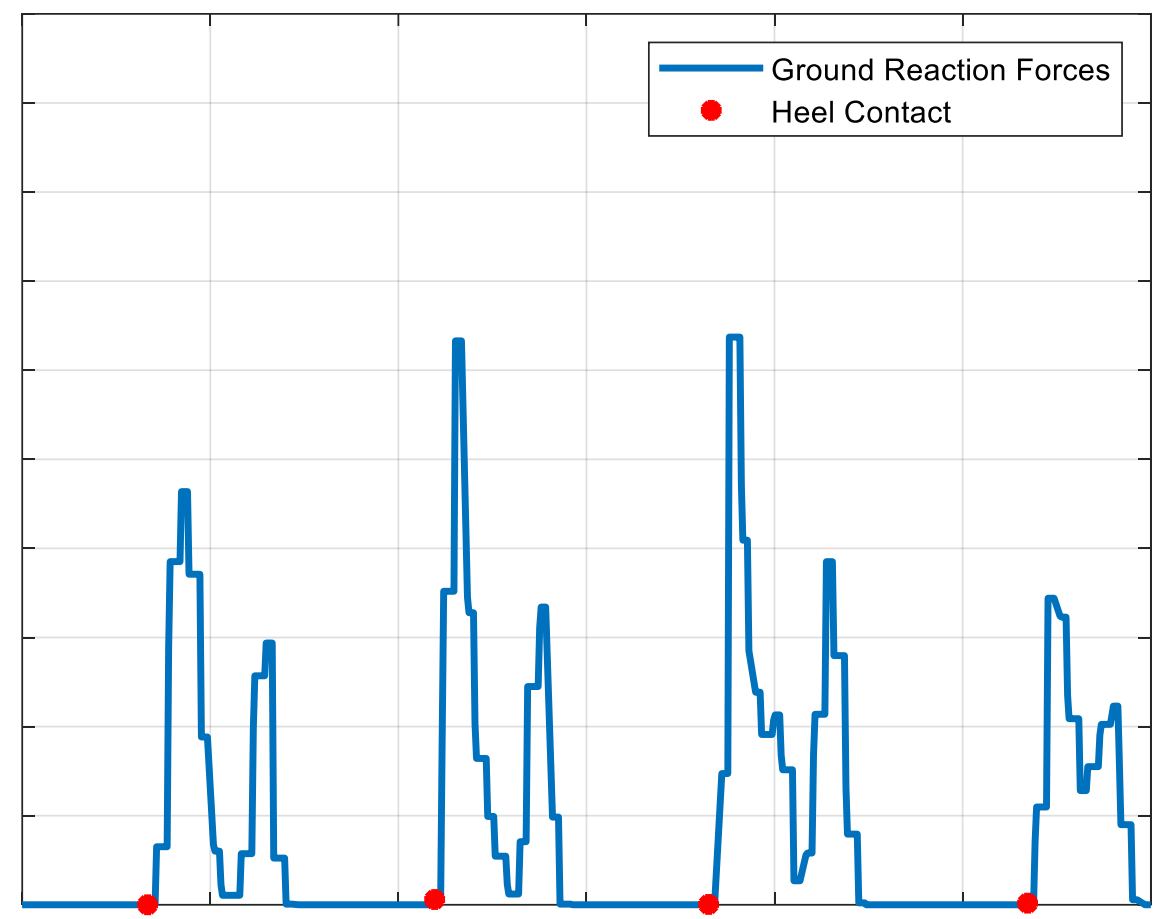

Figure 16. Ground reaction forces recorded using insole piezo-resistive sensors inside prosthetic shoes and detected heel contact times

The timing and samples on different sensors are not the same, meaning the sample showing the HC on GRF signal does not directly show the HC on the EMG, IMU, and IR sensors. Thus, a synchronization method implemented to find the HC time on each sensor. The time is recorded on each sensor and it was used for synchronization. Once $\mathrm{HC}$ is detected on GRF signal, the closest time to HC was calculated on other sensors and it was chosen as the $\mathrm{HC}$ on that specific sensor. This synchronization was done as the following:

$$
T_{H C_{m}}=\min _{n}\left|T_{m}-T_{H C_{G R F}}\right|
$$

Where, $T_{H C_{m}}$ is the time of $\mathrm{HC}$ on sensor $m, T_{m}$ is time on sensor $m$, and $T_{H C_{G R F}}$ is the time of HC on GRF signal.

Finding the $\mathrm{HC}$ time on each sensor ensure synchronization and crucial in avoiding miscalculations. The detected $\mathrm{HC}$ used as the timing for the end of the prediction window, which will allow the control algorithm to predict the intention before prosthetic 
engagement. In the next section, the preprocessing for EMG, IMU, and IR signals are explained.

\subsubsection{Feature extraction}

Following the HC detection, several features extracted from EMG, IMU and IR sensors to quantify the signals and simplify them for the control system. Feature extraction is done in time-domain and frequency-domain to highlight various characteristics of each signal. In this study, a windowed method used for feature extraction. The window ends at the HC to ensure enough time for control system to adapt to change in the terrain.

The EMG signals have a noisy nature and they are susceptible to motion artefact and high-frequency noises. Prior to feature extraction, the noise needs to be removed from the signals. For this goal, a fourth-order bandpass Butterworth filter with high-pass and lowpass bandwidths of 20 and $450 \mathrm{~Hz}$, respectively was applied. Figure 17 shows a sample of the EMG signals before and after artefact removal process. The effect of motion artefact is clearly visible in Figure 17. 

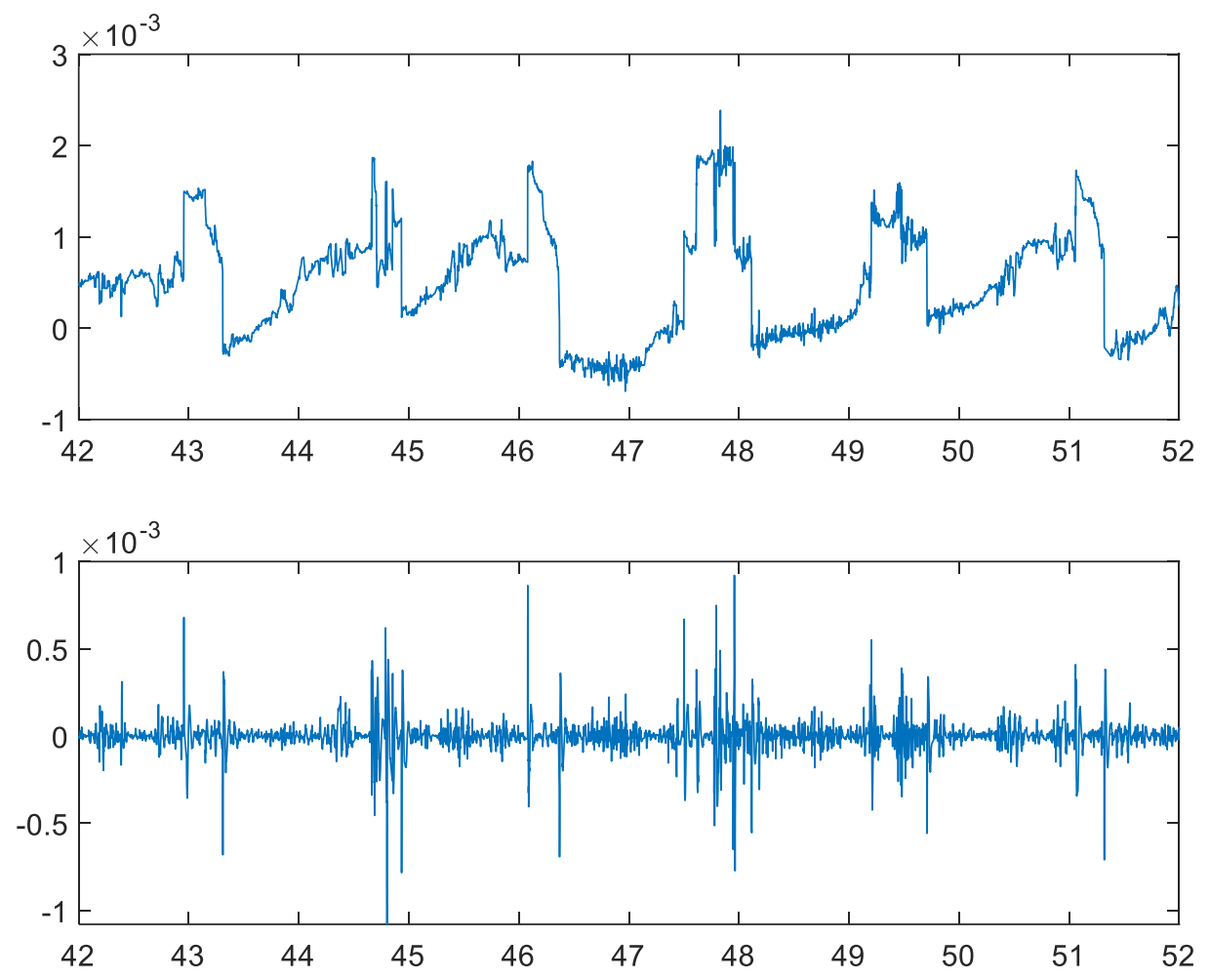

Figure 17. EMG signal from Soleus before and after motion artefact removal of subject 1.

Time-domain and Frequency-domain features extracted from EMG signals for each muscle. Feature extraction is done in a window from $300 \mathrm{~ms}$ before the $\mathrm{HC}$ up to the $\mathrm{HC}$ time. The following features extracted:

- Mean Absolute Value (MAV):

$$
M A V_{m}=\frac{1}{N} \sum_{n=1}^{N}\left|E M G_{m}[n]\right|
$$

Where $\mathrm{N}$ is the length of the window, $E M G_{m}$ is the EMG signal for muscle $m$.

- Variance (Var):

$V A R_{m}=\frac{1}{N} \sum_{n=1}^{N}\left(E M G_{m}[n]-\mu_{m}\right)^{2} ; \mu_{m}=\frac{1}{N} \sum_{n=1}^{N} E M G_{m}[n]$

- Root Mean Square (RMS): 


$$
R M S_{m}=\sqrt{\frac{1}{N} \sum_{n=1}^{N}\left(E M G_{m}[n]\right)^{2}}
$$

- Log Detector (LogDet): estimates the muscle contraction force in logarithmic form as following:

$$
\operatorname{LogDet}_{m}=\exp \left(\frac{1}{N} \sum_{n=1}^{N} \log \left(\left|E M G_{m}[n]\right|\right)\right.
$$

- Wavelength Length (WL) shows the complexity of the signal.

$$
W L_{m}=\sum_{n=2}^{N}\left|E M G_{m}[n]-E M G_{m}[n-1]\right|
$$

- Zero-Crossing (ZC): this measure shows the number of times the EMG signal changes sign and it shows the frequency component of signal in the time domain.

$$
\begin{gathered}
Z C_{m}=\sum_{n=2}^{N} \operatorname{sgn}\left(E M G_{m}[n] . E M G_{m}[n-1]\right) \\
\operatorname{sgn}(X)= \begin{cases}0 & X<0 \\
1 & X \geq 0\end{cases}
\end{gathered}
$$

- Willison Amplitude (WAmp)

$$
\begin{gathered}
W A m p_{m}=\sum_{n=2}^{N} F\left(\left|E M G_{m}[n]-E M G_{m}[n-1]\right|\right) \\
F(X)= \begin{cases}0 & X<\text { threshold } \\
F(X) & X \geq \text { threshold }\end{cases}
\end{gathered}
$$

- Slope Sign Change (SSC) is the number of times the slope of the EMG signal changes sign. 


$$
\begin{aligned}
& S S C_{m}=\sum_{n=2}^{N-1} f(\left(E M G_{m}[n]-E M G_{m}[n-1]\right) \\
&\left.\times\left(E M G_{m}[n]-E M G_{m}[n+1]\right)\right) \\
& f(x)= \begin{cases}0 & X<\text { threshold } \\
1 & X \geq \text { threshold }\end{cases}
\end{aligned}
$$

Eq.

The threshold of $50 \mu V$ used for both $W A m p_{m}$ and $S S C_{m}$ features.

In addition, IMU sensors are preprocessed and features are extracted from them in the same window before the HC. IMU signals used in this study have 3 degrees-of-freedom (DOF) that consists of accelerometer and gyroscope signals in $\mathrm{x}, \mathrm{y}$, and $\mathrm{z}$-axis. The features that are extracted from these signals are mean, maximum value, minimum value, and standard deviation of each signal. The calculation of these features is done as follows:

- Mean:

$$
\mu_{m}^{I M U}=\frac{1}{N} \sum_{n=1}^{N} I M U_{m}[n]
$$

- Maximum:

$$
\max _{n}^{\mathrm{IMU}}=\max \left(I M U_{m}\right)
$$

- Minimum:

$$
\max _{m}^{I M U}=\min _{n}\left(I M U_{m}\right)
$$

- Standard deviation:

$$
\sigma_{m}^{I M U}=\sqrt{\frac{1}{N} \sum_{n=1}^{N}\left(I M U_{m}[n]-\mu_{m}^{I M U}\right)^{2}}
$$


Prior to feature extraction, a $4^{\text {th }}$-order low-pass Butterworth filter with a cutoff frequency of $20 \mathrm{~Hz}$ applied to remove high-frequency noises.

Finally, the IR sensors reading at the $\mathrm{HC}$ added to the feature set to facilitate the detection of physical obstacle and improve the performance. Considering there are 10 channels of EMG and 5 IMU sensors and one IR sensor, in the end, there are 60 EMG features, 120 IMU features and, 1 IR features. This results in a feature vector with 181 dimensions.

\subsubsection{Prediction model}

To provide adaptive control and ensure a smooth transition between different terrain types it is important to predict the change before it happens. This would allow the system to change parameters and ensure smooth transition. For this purpose, a machine-learning algorithm developed to fuse multiple information, classify, and predict the terrain type of the next step from the current HC.

\subsubsection{Feature selection}

It is important to reduce the feature dimensions to prevent dimensionality problem and reduce the complexity by removing the correlated and irrelevant features. This will also reduce complexity and calculation time. Thus, an ensemble learning method based on decision trees implemented. Extra Tree Classifier (ETC), which randomizes the decisions and subsets the data to minimize over-learning and over-fitting, applied to the data. Decision tree-based methods work using the basic idea of using multistage approach to break up a complex decision into a union of several simpler decisions and assigns importance to the features that have been used [120]. The ETC classifier uses an extreme randomization process to draw the splits which results in reduced complexity and increased speed [121]. The feature importance calculated and number of features to be used in the 
prediction was investigated in this study. The feature selection is done on training data with a supervised learning approach. An ETC trained using training data with and best feature subset is chosen according to their importance in the trained ETC. The number of selected features investigated to find the best design that would provide a high accuracy without overfitting. The feature selection is done on subject-dependent basis to ensure the subject variability is considered. As a result, the final feature dimension is expected to vary from subject to subject.

\subsubsection{Gradient Boosted Tree (GBT)}

Decision trees use a tree-like model for decision-making and is a popular tool in regression and classification. The basic idea of decision trees arises from the approach of breaking up a complex decision into a union of several simpler decisions [120]. This machine learning technique is easily visualized and interpreted. In addition, it does not require feature normalization and works well with datasets containing mixture of features. However, decision trees can easily overfit the machine-learning problem. Usually to avoid overfitting an ensemble of trees implemented for better generalization.

Gradient Boosted Trees (GBT) build the prediction model using a series of small decision trees. Each tree attempts to correct errors from the previous stage. Figure 18 illustrates the general outline for GBTs. GBT complexity is controlled using a learning rate, which controls how hard each new tree tries to correct the remaining mistakes from the previous round. High learning rate results in more complex trees, while lower learning rate training simpler trees. 


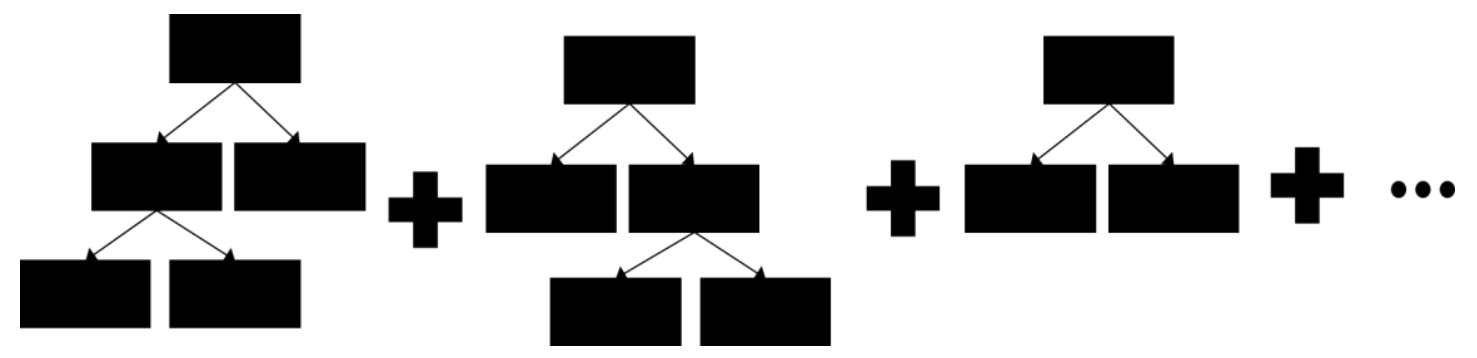

Figure 18. A general overview of gradient boosted trees.

GBMs often work well for many problems, the prediction model requires only modest memory, and it is fast. GBMs also do not require careful normalization of features and handles mixture of features well. However, inclusion of the gradient boosting method increases the complexity and makes it hard to interpret. In this study, GBM method implemented without feature normalization to investigate terrain prediction performance.

\subsubsection{Pseudo real-time testing}

Aim of this study is to present a real-time adaptive control system for a powered prosthesis. In this study, a pseudo-real-time method implemented to test the real-time terrain prediction. Figure 19 shows the flowchart of the pseudo-real-time adaptive control system. 


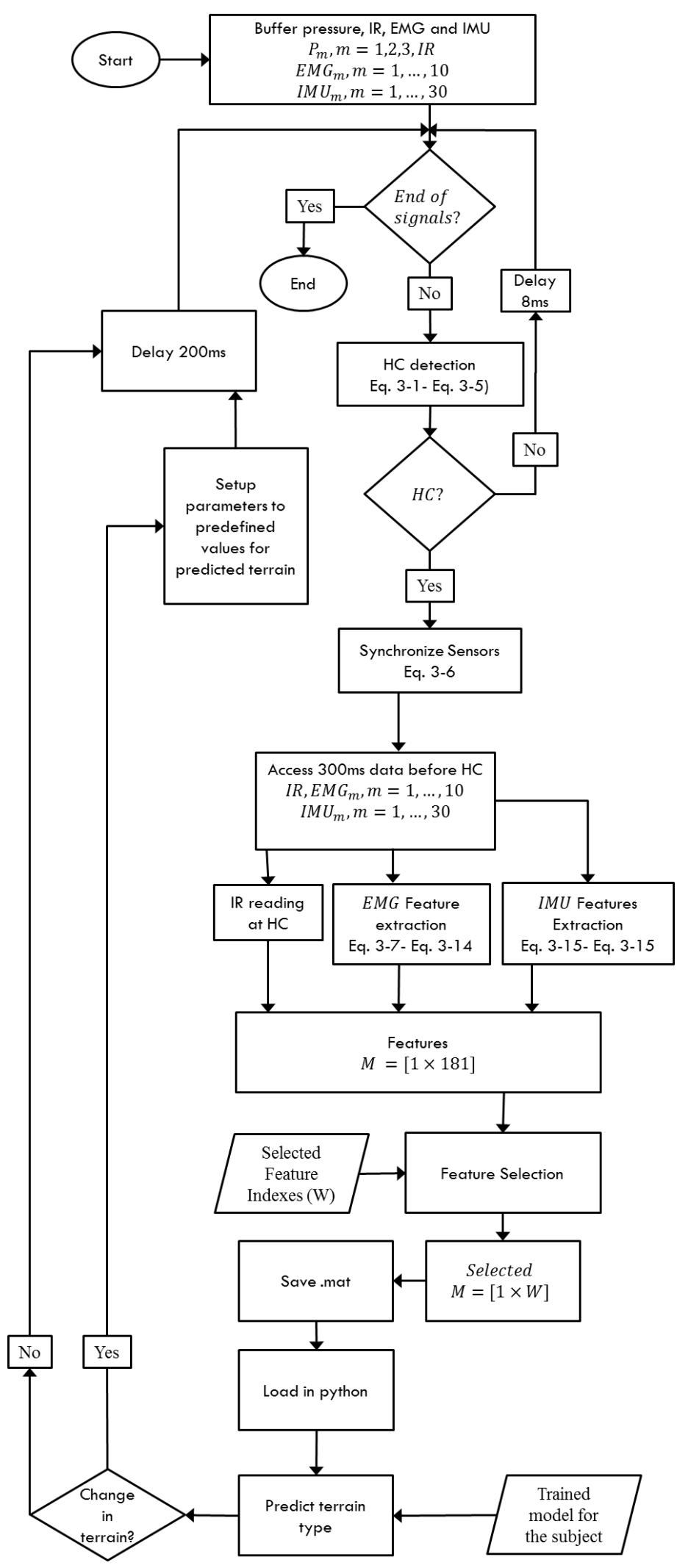

Figure 19. Flowchart for the pseudo-real-time terrain mode prediction and adaptive control for powered prosthetic ankle optimization. 
The real-time system uses the trained model and uses the model to predict each step before prosthetic HC. The system starts with loading the data to a buffer. The second step of the flowchart is HC detection, where the system loads the last $200 \mathrm{~ms}$ data from the pressure data on the prosthetic foot and uses Eq. 3-1 through Eq. 3-5 to find the HC. The $\mathrm{HC}$ loop is repeated until a new $\mathrm{HC}$ is detected. Once the $\mathrm{HC}$ is detected the system find the exact time of the $\mathrm{HC}$ on pressure sensor and matches the EMG and IMU sensors timing with that. After this synchronization part, the system extracts features from IR, EMG and IMU signals using Eq. 3-7 through Eq. 3-15 and builds a vector for testing. Then input feature space is reduced using selected features in the training. Then reduced feature vector is input to the trained model and terrain type gets a prediction. Following the prediction of the state for next step, the system checks to see whether the label is different from the current one. In case of change in the terrain type, system automatically changes the stiffness and power parameter to the pre-tuned values by the prosthetic clinicians. The system repeats this approach until it reaches to the end of the signals.

\subsubsection{Evaluation}

The prediction system was based on supervised machine learning. The following measures are introduced for evaluation of the system performance on predicting the terrain type. The prediction system included four classes of level walking, stair ascent, stair descent and, turning.

$$
\begin{array}{lc}
\text { Sensitivity }=\frac{T P}{T P+F N} \times 100 \% & \text { Eq. } \\
\text { Specificity }=\frac{T N}{T N+F P} \times 100 \% & \text { Eq. }
\end{array}
$$




$$
\begin{array}{cc}
\text { Precision }=\frac{T P}{T P+F P} \times 100 \% & \text { Eq. } \\
\text { Accuracy }=\frac{T P+T N}{T P+F P+T N+F N} \times 100 \% & \text { Eq. } \\
F 1-\text { score }=\frac{2 \times \text { Precision } \times \text { Sensitivity }}{\text { Precision }+ \text { Sensitivity }} \times 100 \% & \text { Eq. }
\end{array}
$$

Where, TP is the number of positive events correctly predicted as positive, FP is the number of negative events falsely predicted as positive, $\mathrm{TN}$ is the number of the negative event correctly predicted as negative, $\mathrm{FN}$ is the number of positive events falsely predicted as negative.

The accuracy measure shows the overall performance over all of the testing samples. Recall or true positive rate show the accuracy of getting positive event correct. Precision is the positive predicted value rate. F1-score is harmonic mean of precision and recall. It is worth noting that the classes investigated in this study are not balanced meaning there are more level ground samples compared to transition to uneven terrains and if the evaluation gets limited to accuracy it will fail to show the full picture. For this purpose, precision, recall, and F1-score used in the evaluation. 


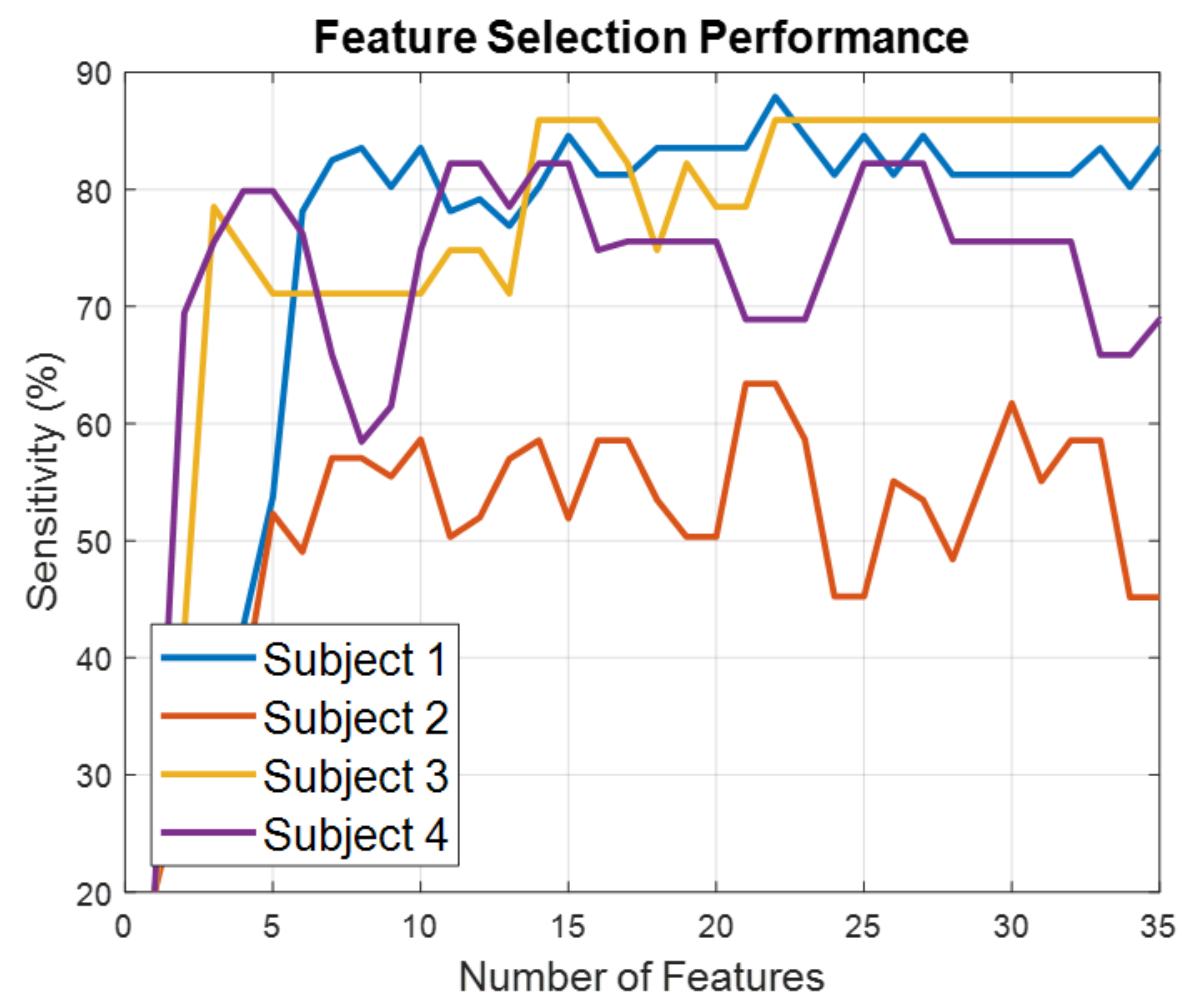

Figure 20. Feature selection results.

\subsection{Results}

Data processing is done in MATLAB and Python platforms. The data labeling was done during data collection and compared to expert labeling according to waveforms and ensure accuracy. HC detection was validated by comparing to visual detection and resulted in $100 \%$ accuracy. Table 4 shows the results of $\mathrm{HC}$ detection validation.

Table 4. Automatic HC detection results

\begin{tabular}{|c|cccc|}
\hline Subject & Total HCs & Missed HCs & Average Latency & Accuracy \\
\hline 1 & 951 & 0 & $0.3 \mathrm{~ms}$ & $100 \%$ \\
\hline 2 & 747 & 0 & $13.3 \mathrm{~ms}$ & $100 \%$ \\
\hline 3 & 504 & 0 & $0 \mathrm{~ms}$ & $100 \%$ \\
\hline 4 & 534 & 32 & $12 \mathrm{~ms}$ & $94 \%$ \\
\hline
\end{tabular}


The maximum number of features investigated for each subject from 5 up to 50 features. Importance of number of features investigated using a 10-fold cross-validation scheme and independent testing. Feature selection is done using Extra Tree Classifier. Figure 20 shows the accuracy for feature selection. The feature space dimension is chosen to provide the highest average cross-validation accuracy for training. For example, according to Figure, 22 features selected for subject 1 . Names of the selected features for each subject is listed in Table 5.

Table 5. Selected features using feature importance of extended tree classifier.

\begin{tabular}{|c|c|c|}
\hline Subject & $\begin{array}{l}\text { Number of } \\
\text { features }\end{array}$ & Selected Features \\
\hline 1 & 22 & 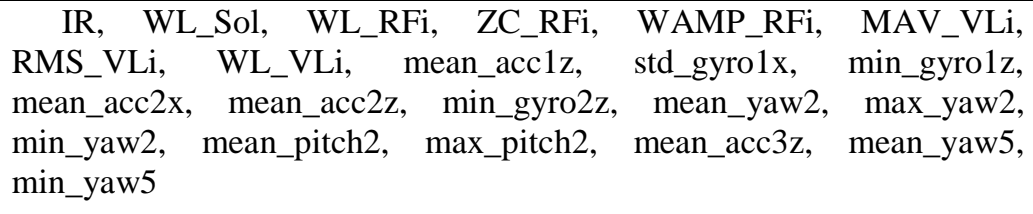 \\
\hline 2 & 21 & $\begin{array}{l}\text { IR, MAV_VLi, RMS_VLi, WL_VLi, mean_yaw1, max_yaw1, } \\
\text { min_yaw1, mean_acc2z, mean_gyro2z, std_gyro2z, mean_yaw2, } \\
\text { min_yaw2, max_acc3y, std_gyro3x, std_yaw3, min_yaw4, max_gyro5x, } \\
\text { std_gyro5y, mean_yaw5, max_yaw5, min_yaw5 }\end{array}$ \\
\hline 3 & 14 & $\begin{array}{l}\text { IR, WL_TA, RMS_Sol, mean_yaw2, max_yaw2, min_yaw2, } \\
\text { mean_acc4z, max_acc5z, mean_gyro5y, mean_yaw5, max_yaw5, } \\
\text { mean_pitch5, max_pitch5, min_pitch5 }\end{array}$ \\
\hline 4 & 11 & $\begin{array}{l}\text { IR, RMS_Sol, WL_Sol, mean_gyro2y, max_yaw2, min_yaw2, } \\
\text { mean_acc4z, std_acc4z, std_pitch4, max_acc5z, min_pitch5 }\end{array}$ \\
\hline
\end{tabular}

The selected features for each subject used to train classifiers. The training data divided into training and validation subsets and best classifier parameters was investigated. The validation subset used to ensure that it is not overfitting the training data and will be generalizable to unseen data. 
The trained classifiers for each subject saved and it was tested in a pseudo-real-time approach on multiple recordings that were not used in the training part. The pseudo-realtime approach starts by initializing a 200ms window of data and buffering GRF, IR, EMG and IMU signals. From the buffered data, the method searches for an $\mathrm{HC}$ and repeats the process until an $\mathrm{HC}$ is detected. When $\mathrm{HC}$ is detected features are extracted from $300 \mathrm{~ms}$ before HC up to HC. Figure 21, shows a sample for GRF and EMG signals used in this study that was processed in real-time and HCs are shown. The highlighted parts in Figure shows the $300 \mathrm{~ms}$ window where the feature extraction is done and two samples of transition to stair ascend and a level ground sample is highlighted. It can clearly be seen from Figure 21 that the EMG activity is higher in amplitude and frequency for TA, Sol, VL, and RF on intact side, which was chosen in the feature selection part (Table 5).

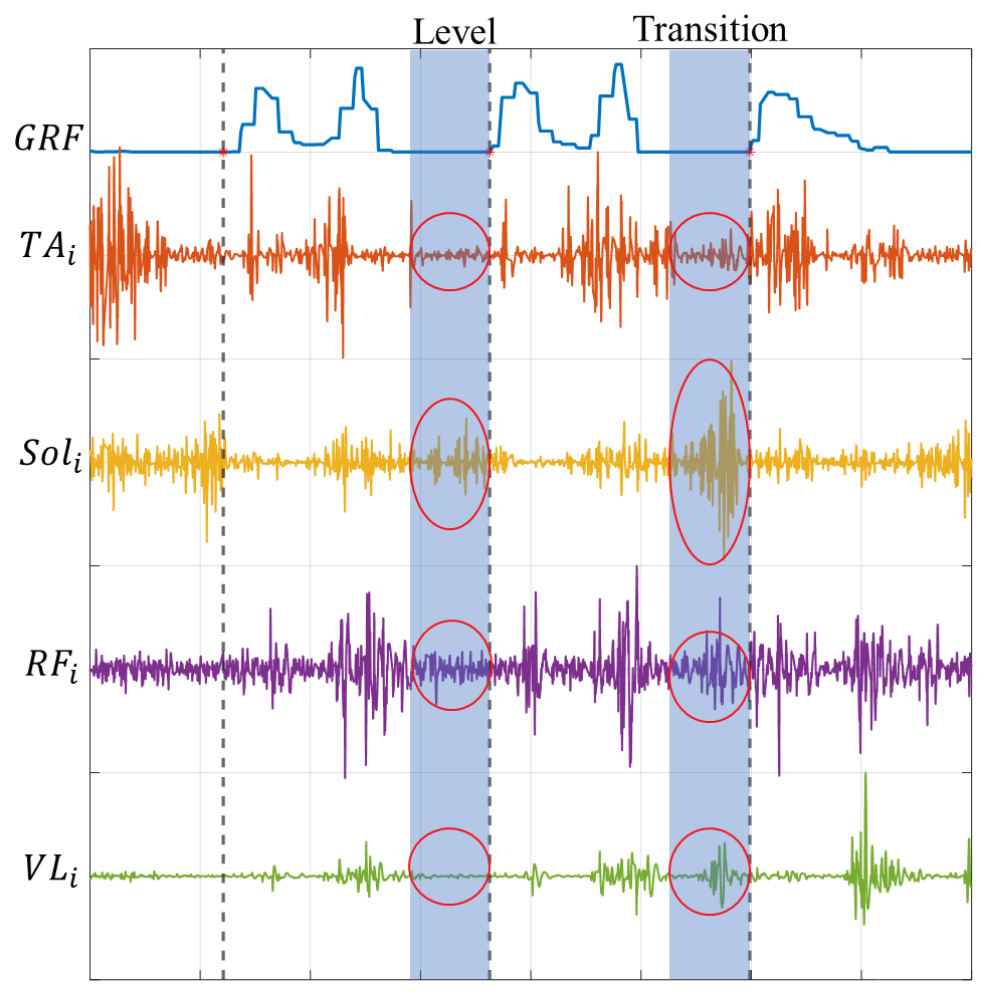

Figure 21. A sample of GRF and EMG signals with detected HCs for two level terrain gait cycles followed by a stair ascend step. The transition and one level terrain sample are highlighted in blue. 
Figure 22 shows the distribution of sample features that have been selected by ETC method. IR feature showed the lower distribution for stair ascend and turning cases while it was saturated for level ground and stair descend. Gyroscope data for stair down shows a lower distribution, which highlight the slowing down. Accelerometer distribution was lower for stair descent and ascent. EMG activity increased in non-level terrains and the biggest meaningful increase was observed for stair ascending.
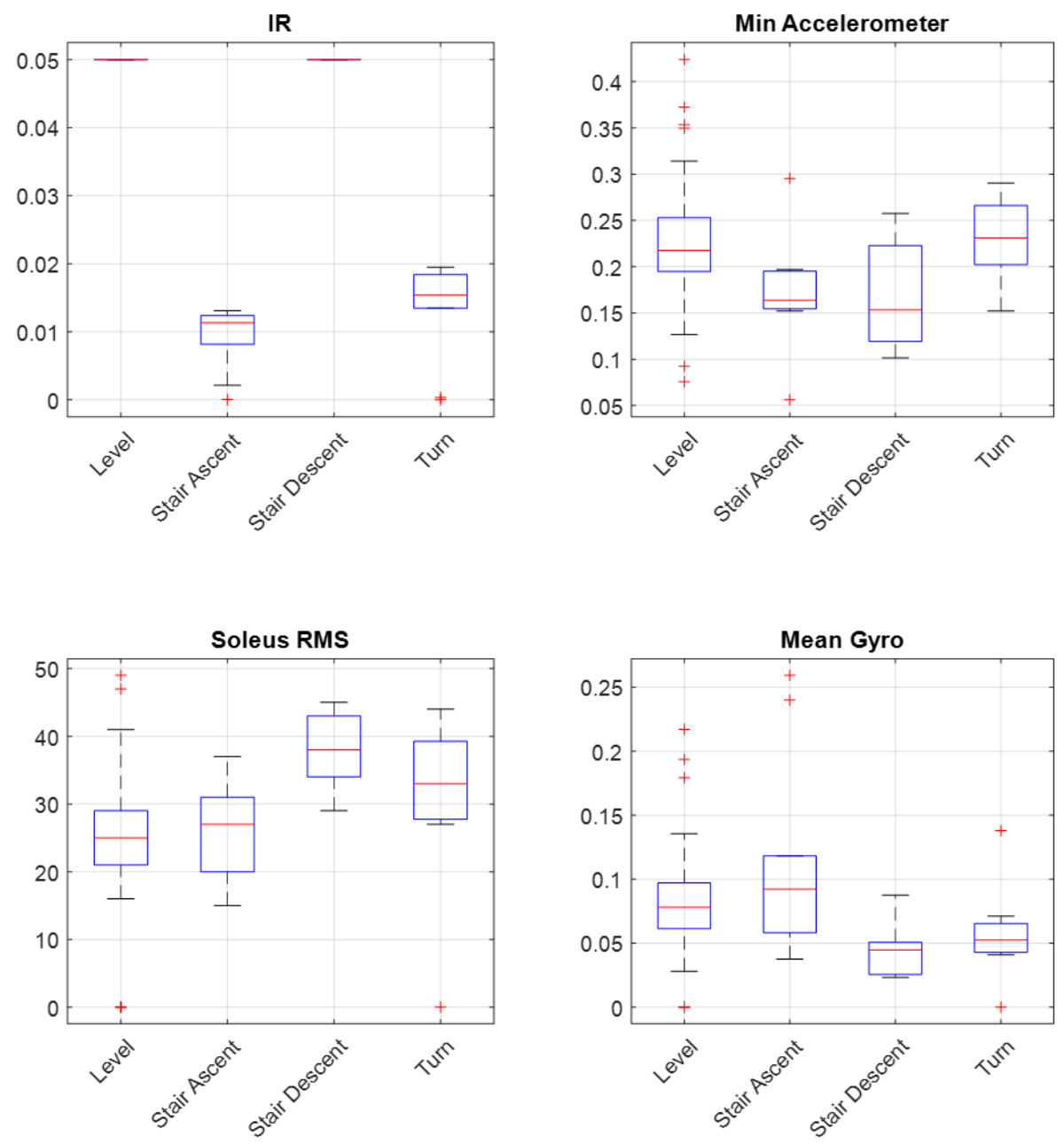

Figure 22. Distribution of selected features for four different classes investigated in this study. (a) IR distance reading at $\mathrm{HC}$ for subject 4; (b) maximum for gyro signal in z-axis on the intact hip of subject 2; (c) minimum for accelerometer in y-axis on amputee's hip for subject 1; (d) RMS for intact Soleus for subject 3. 
The combination of different sensors fusion methods compared to find the least complexity for terrain prediction. Figure 23, shows the accuracy, sensitivity, specificity results for pseudo-real-time testing for each subjects to detect the change in terrain. Sensor fusion significantly performed better than single sensor prediction ( $\mathrm{p}$-value $<0.05)$. However, there was no significant difference between all sensors combination versus combination of IMU and IR.
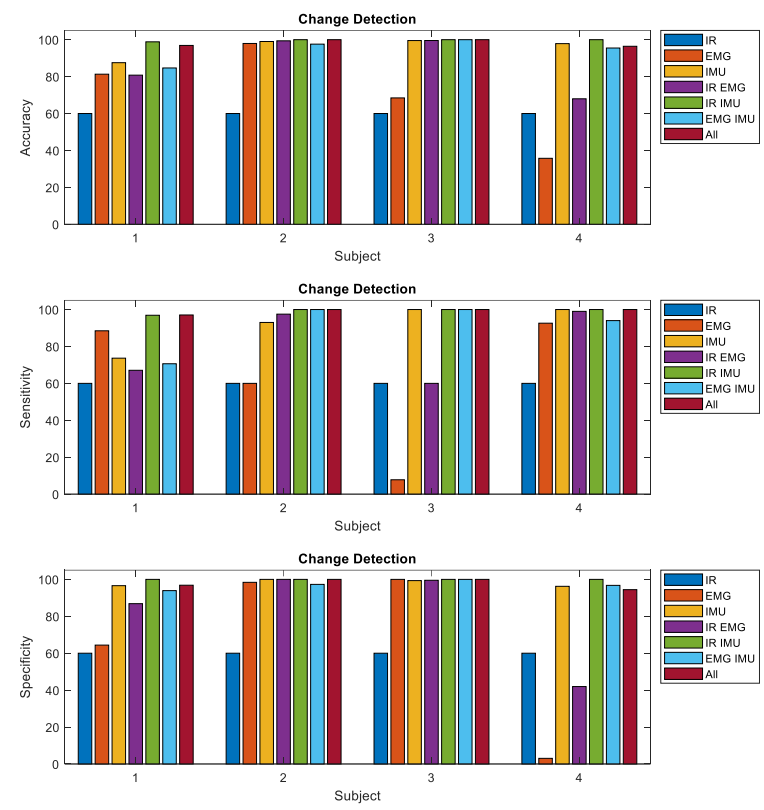

Figure 23. Pseudo-real-time testing results for detection of the change in locomotion mode.

Figure 24, Figure 25, and Figure 26 illustrates the comparison of sensor fusion methods for prediction $\mathrm{f}$ stair ascent, stair descent, and turning examples respectively. Similar to detection part, IR and IMU fusion performed significantly higher than simpler methods and it did not result in a significant difference with other more complex fusions. 

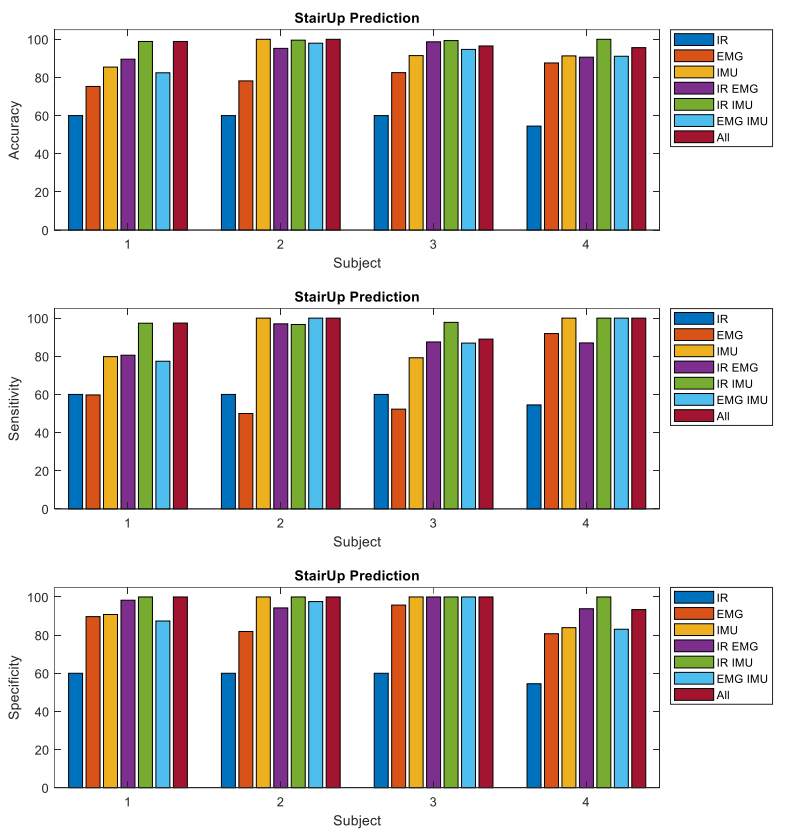

Figure 24. Pseudo-real-time testing results for prediction of stair ascent.
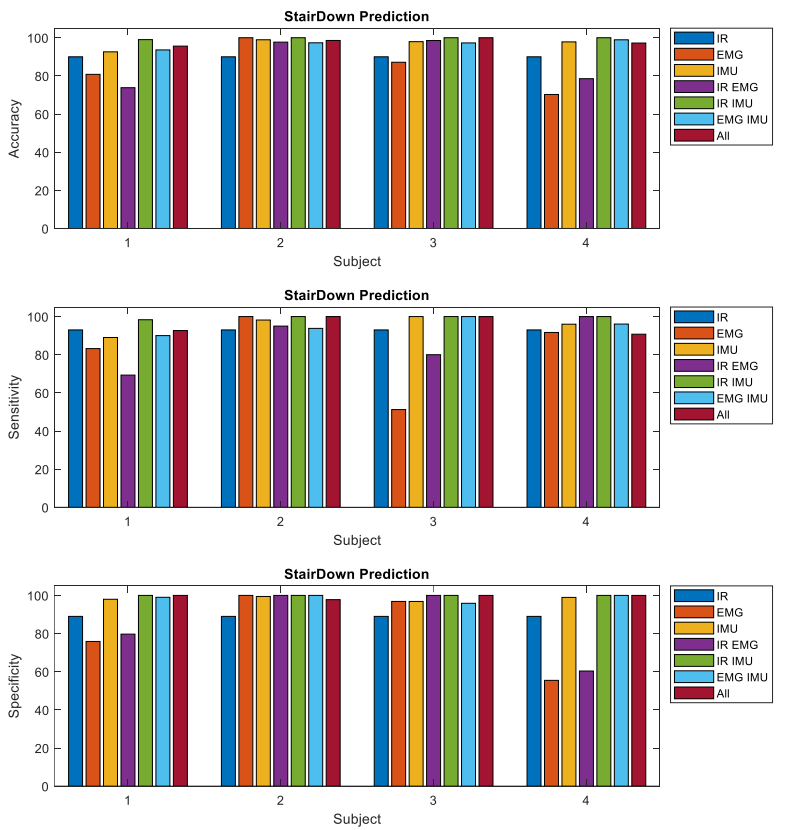

Figure 25. Pseudo-real-time testing results for prediction of stair descent. 

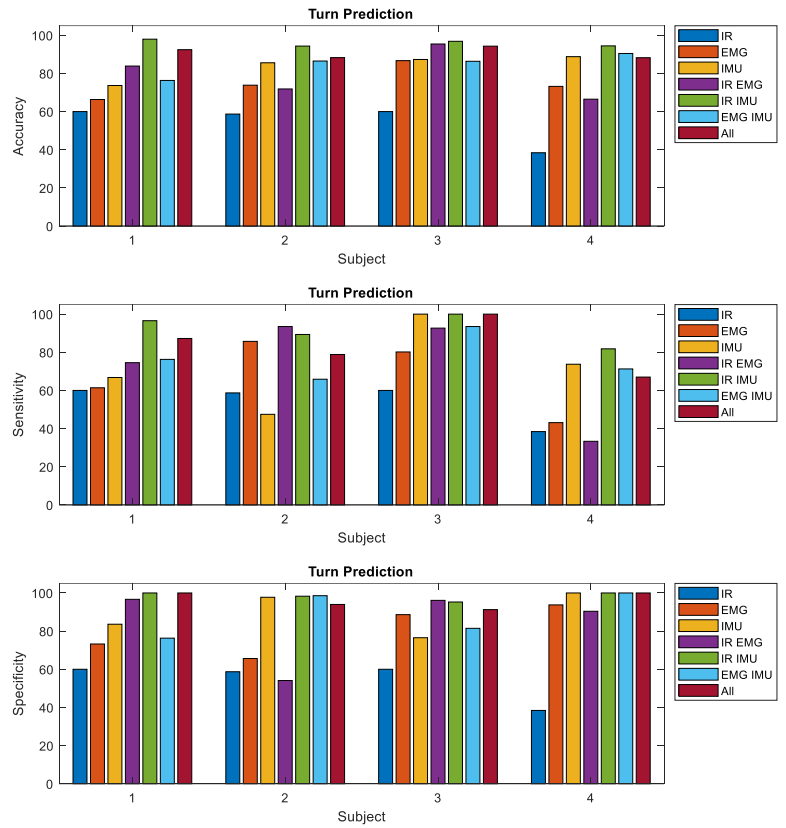

Figure 26. Pseudo-real-time testing results for prediction of turn.

The confusion matrix for pseudo-real-time testing is shown in Figure 27 for IMU and IR fusion method. Fusion of IR and IMU resulted in a significantly higher accuracy, sensitivity, and specificity for each locomotion mode prediction and the change detection. This fusion method is easier to implement and add to prosthetic compared to EMG and the processing is less complex. 

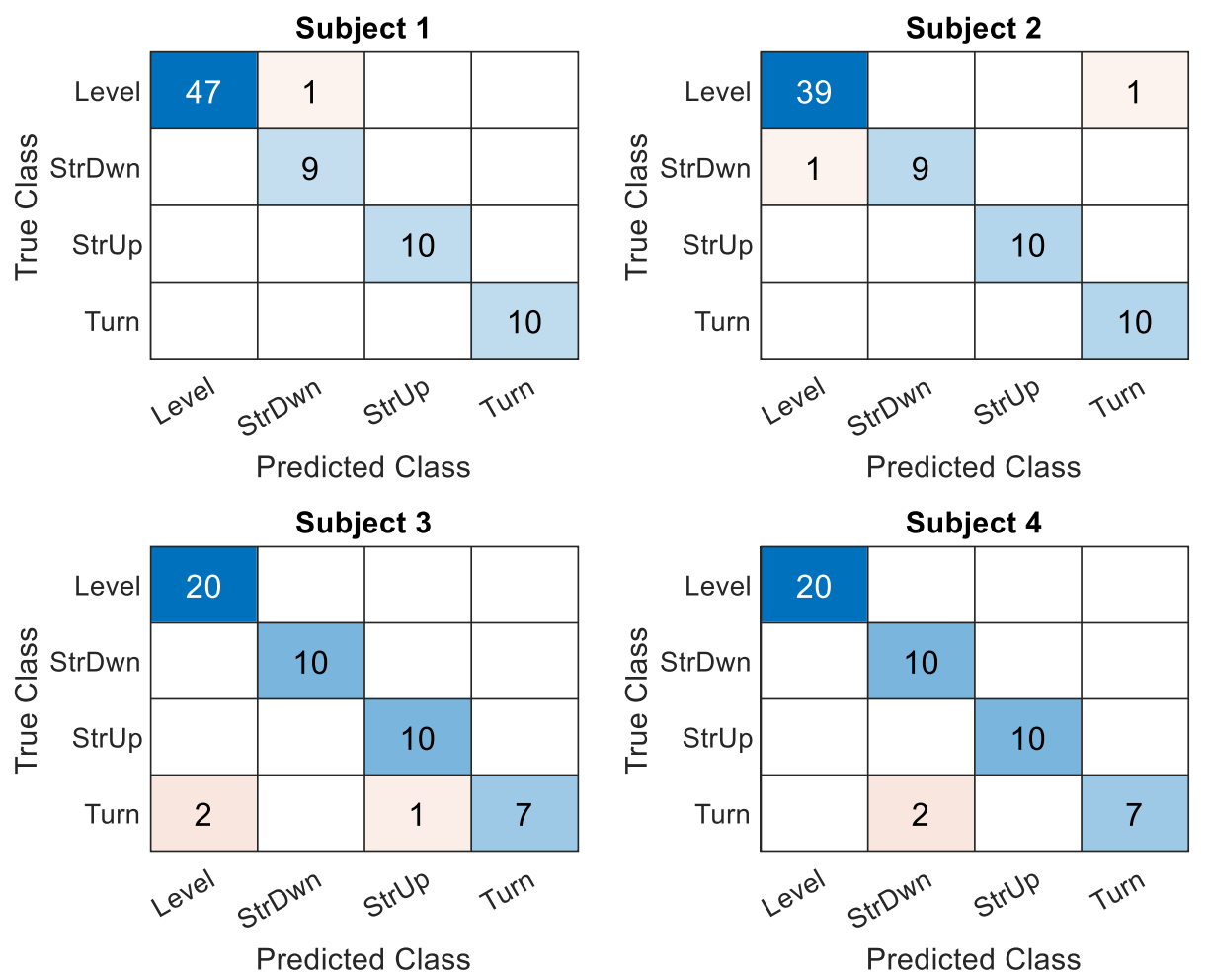

Figure 27. Confusion matrix for pseudo real-time testing of terrain prediction on four subjects.

Table 6. shows the performance measures of accuracy, sensitivity, and specificity for the four terrains investigated in this study. The evaluation of real-time simulation resulted in accuracy of more than $94 \%$ for prediction of the change in terrain.

Table 6. Accuracy, Sensitivity and, Specificity measures for change and terrain prediction for level terrain, stair ascent (SU), stair descent (SD), and turn (T).

\begin{tabular}{ccllcccccc}
\hline Subjects & Accuracy & \multicolumn{4}{c}{ Sensitivity } & \multicolumn{5}{c}{ Specificity } \\
\hline & & L & SU & SD & T & L & SU & SD & T \\
$\mathbf{1}$ & 98.7 & 96.6 & 100 & 100 & 100 & 100 & 100 & 100 & 100 \\
$\mathbf{2}$ & 97.1 & 96.6 & 100 & 100 & 100 & 97.5 & 100 & 100 & 100 \\
$\mathbf{3}$ & 94 & 93.1 & 100 & 100 & 87.5 & 100 & 100 & 94.4 & 100 \\
$\mathbf{4}$ & 95.9 & 100 & 100 & 100 & 77.7 & 100 & 89.4 & 100 & 100 \\
\hline
\end{tabular}

\subsection{Discussion}

This study successfully showed the feasibility of predicting terrain type using a sensor fusion technique for real-time adaptive control of PPA. The proposed method fuses 
information on various sensors types including multi-channel physiological, kinetic, and kinematic information and presented a phase-dependent terrain type prediction control system. The results of the study obtained from two simulated subjects (Subject 1,2) and two transtibial amputees (Subject 3,4) while they operated a PPA system. The proposed method resulted in up to 98.7 percent accuracy for prediction of four terrains of level, stair ascent, stair descent, and turning.

The phase-dependency approach was chosen to ensure enough time for the control system to adapt to change when it happens. BIOM PPA that was used in the study works on a phase-based control system that imitates the lost muscles on lower leg and provides positive power in the HO. As the PPA system provides the net-positive power at the HO phase of prosthetic gait it was concluded that the ability to predict the terrain change at $\mathrm{HC}$ would be optimal for control system. The HC detected using a custom-built wearable GRF system embedded with insole pressure sensors. HC time used as the end of the processing window and other sensors synchronized and buffered 300ms before HC up to HC.

In this study, neural information from lower leg muscles on both intact and prosthetic side was used to quantify the intention and use it for prediction. Ten channels of EMG signals collected from the subjects while they walked on different terrains and multiple time-domain and frequency-domain features calculated on a windowed signal from 300ms before $\mathrm{HC}$ up to the HC. In addition, five IMU sensors placed on both legs on the intact ankle, both knees, and both hips. Each IMU consisted of 3 DOF accelerometer and gyroscopes in $\mathrm{x}, \mathrm{y}$, and $\mathrm{z}$ directions. Like EMG, features from low-pass filtered IMU signals are calculated in a window from $300 \mathrm{~ms}$ before HC up to HC. Finally, an infrared distance sensor was placed on the prosthetic toe for obstacle detection and the value at $\mathrm{HC}$ 
was used. The choice of IR distance sensor was done to include knowledge from the environment and the results showed that this information is very useful for terrains that lead to a physical barrier, which in this case included stair ascent and turning.

Features selection was done to investigate the efficacy of various sensors and reduce the complexity of the prediction system. As it was expected, IR sensor was saturated in absence of a physical obstacle and had smaller value otherwise. This provided a great discriminatory feature between stair ascend and turning versus stair descend and level ground. This effect can clearly be seen in distribution of the IR feature in Figure 22.

The selection of features showed that the muscles on the intact side provide the most useful information regarding the change in the terrain. This effect happens as a result of investigating the change before it happens on the prosthetic side. Even though there is no change in the terrain at the $\mathrm{HC}$ of prosthetic foot, but subject's muscle activity increases on the intact side as the intact side starts to transition to new terrain. This is clearly seen from Figure 21, where the EMG activity increases in the transition step compared to the level ground steps prior to it. In addition, this relates to the fact that during prosthetic gait users tend to rely more on the intact side and as result increased activity on this side.

IMU features also showed a meaningful difference in their distribution for various terrains. Generally, there was a lower distribution in accelerometer data which happens as a result of slowing down and change in the accelerometer and gyroscope on the prosthetic limb. The slowing down was more dominant on simulated subject as they were inexperienced to prosthetic use compared to amputees. On the intact side, as the limb prepares to start transitioning the moments tend to increase and the IMU data showed this behavior. Biomechanically, prosthetic gait when transitioning into stairs (ascent or descent) 
moments on the prosthetic limb reduce as a result of slowing and down increase on the intact side which can quantified using the IMU data.

The first major challenge in adaptive control of PPAs is the ability to detect change from level terrain before it happens. The second challenge is to predict the non-level terrain from other non-terrain types. The current shortcoming for PPAs is the lack of adaptivity to different terrains and because of that the setup is constant throughout the day.

To resolve this shortcoming a machine learning-based models developed to investigate the feasibility of improving the current control systems by making them adaptive. In this study, a Gradient Boosted Tree classifier was trained and simulated real-time testing resulted in an accuracy of up to $98.7 \%$ and sensitivity and specificity of up to $100 \%$. These results ensure that the first challenge of detecting the change is feasible.

The results prove the hypothesis of using a sensor fusion method for data before even entering a new terrain it is possible to predict the change. However, to be able to adaptively control the PPA system it is crucial to predict the type of new terrain as they require different assistance levels. For example, going up the stair requires more power and less stiffness while going down needs less power and more stiffness. Using GBT classifier terrain types predicted and confusion matrix for the actual terrain versus the predicted one was shown in Figure 27.

Throughout the day it is possible to walk toward obstacles or stairs that might lead the algorithm to detect stair descend and increase the power. However, it is likely these events might not lead to stair descend. To simulate these situations and test the system subjects walked toward the stairs but did not proceed to go up but turned around continued walking 
on level ground. The results of the simulation resulted in 2 samples of turning getting mislabeled as stair ascent for subject 4 and 3 misclassification for subject 3 .

The results of the study showed a high accuracy of up to $98.7 \%$ for prediction terrain for four different terrains. The study used a sensor fusion method to incorporate IMU and IR signals to predict the change before it happens and adapt the PPA system to the new terrain. The results of this study prove that it is feasible to use various sensors and fuse their information to predict changes in the terrain type in real-time. Implementation of the present method will result in smooth transitions between terrains and optimal assistance for different intentions.

\subsection{Conclusion}

In this chapter, a novel pseudo-real-time approach was presented for prediction of different terrain types and detecting the changes. The changes in terrain were detected using a machine learning classifier and a control system proposed to adapt the PPA to change in the terrain and improve gait and balance for amputee users. 


\section{CHAPTER 4 CONCLUSION}

In this study, a novel optimization and control scheme presented for powered lower limb prosthesis. The study included two major parts of optimized tuning and adaptive control. The proposed methods utilize wearable wireless body area sensor networks to collect physiological, kinetic and kinematic information from the residual limbs and transform the collected data into meaningful categories to be used in optimization and control.

The optimized tuning part focuses on the prosthetic parameter setup. The main parameters for the BIOM leg are the stiffness and power parameters. These parameters control the amount of assistance that PPA provides and the resistance in the prosthetic joint. Currently, the parameter tuning is done by prosthetic clinicians, which lack quantitative measure and objectivity. The current methods rely on the experience of the clinician and feedback from the user. This process usually requires multiple appointments until the best setup can be determined.

The proposed alternative method used various sensors from the residual limbs to replace the observation-based tuning with an evidence-based approach. The proposed method collected EMG signals from the muscles on residual limbs and modeled the metabolic energy. The ultimate goal is for the PPA system to minimize the metabolic energy to provide the most natural assistance. The energy model optimization for various parameter combinations resulted in a comparable value with expert values that showed the feasibility of using a smart technique for this purpose. However, due to a having so many possible combinations for the parameters it is not feasible to collect all of them which would take more than 14 hours of data collection. To resolve the issue a Nelder-Mead Simplex (NM) optimization method proposed which complemented with a Latin Hypercube Sampling 
(LHS). The proposed method optimized the energy expenditure model using a smaller number of combinations and was able to do the tuning in less than 12 minutes.

The second and most important challenge in PPA control is adaptive prediction of the terrain. Operating PPA systems have shown to lower the energy expenditure and be very effective in mimicking natural gait in level terrains. However, amputees face various terrains such as stairs, ramps and obstacles throughout daily life and can be challenging to operate PPAs in these situations. Having impedance-controlled parameters allows PPA systems to provide different assistance on different terrains. Even though current systems are able to provide varying assistance depending on the terrain type, they lack an accurate adaptive terrain prediction control system to provide adaptive assistance. In chapter 3 , a real-time adaptive control system presented to resolve this challenge.

The adaptive control system used a phase-dependent approach to predict the upcoming terrain type on the heel contact (HC) for prosthetic leg. The ability to predict the changes in terrain type at the $\mathrm{HC}$ allows the control system to change the parameter setup for the PPA to have optimal assistance in non-level terrains. The proposed method used EMG signals from lower limb muscles, multiple IMU signals on knees, hips and intact ankle, ground reaction forces and, infrared distance sensors. The collected information was processed using sensor fusion techniques and modelled using a machine-learning-based classifier to predict different terrains. Comparison of various sensors compared and it was observed that addition of more sensors does not result in a significant difference and the best combination with smallest complexity was chosen as combination of IR and IMU sensors. The system evaluated using an independent testing data in pseudo-real-time 
approach. The results of the study showed up to $98.7 \%$ accuracy for prediction of stair ascend, stair descend, and turning from level terrain at $\mathrm{HC}$ time.

\subsection{Future direction}

Future works will focus on implementing the adaptive control in real-time and extend to different terrains such as ramps or are complex geometries and natural terrains like walking on sand or hiking outdoors. In future studies, the changes throughout the day and from day to day will be investigated and included to have a more comprehensive control system.

In addition, it is necessary to study the electrode implantation inside the socket and over the limb to be able to embed the sensors with current PPA technologies, which would allow long-term use and facilitate studying real-life situations that can be tested outside the lab environment. Furthermore, it is beneficial to investigate optimized socket design and fitting to improve the comfort and prevent damages as a result of long-term use on the residual limbs. 


\section{REFERENCES}

1 Eilenberg, M.F., Geyer, H., and Herr, H.: 'Control of a powered ankle-foot prosthesis based on a neuromuscular model', IEEE transactions on neural systems and rehabilitation engineering, 2010, 18, (2), pp. 164-173

2 Dillingham, T.R., Pezzin, L.E., and MacKenzie, E.J.: 'Limb amputation and limb deficiency: epidemiology and recent trends in the United States', Southern medical journal, 2002, 95, (8), pp. 875-884

3 Wrobel, J.S., Mayfield, J.A., and Reiber, G.E.: 'Geographic variation of lowerextremity major amputation in individuals with and without diabetes in the Medicare population', Diabetes care, 2001, 24, (5), pp. 860-864

4 Lavery, L.A., Ashry, H.R., Van Houtum, W., Pugh, J.A., Harkless, L.B., and Basu, S.: 'Variation in the incidence and proportion of diabetes-related amputations in minorities', Diabetes care, 1996, 19, (1), pp. 48-52

5 Lavery, L.A., Ashry, H., Armstrong, D., and Pugh, J.: 'Diabetes-related lowerextremity amputations disproportionately affect Blacks and Mexican Americans', Southern medical journal, 1999, 92, (6), pp. 593-599

6 Van Houtum, W., and Lavery, L.: 'Regional variation in the incidence of diabetesrelated amputations in the Netherlands', Diabetes research and clinical practice, 1996, 31, (1-3), pp. 125-132

7 Van Houtum, W.H., Lavery, L.A., and Harkless, L.B.: 'The impact of diabetesrelated lower-extremity amputations in The Netherlands', Journal of Diabetes and its Complications, 1996, 10, (6), pp. 325-330

8 Feinglass, J., Kaushik, S., Handel, D., Kosifas, A., Martin, G.J., and Pearce, W.H.: 'Peripheral bypass surgery and amputation: northern Illinois demographics, 1993 to 1997', Archives of Surgery, 2000, 135, (1), pp. 75-80

9 Boyle, D., Parker, D., Larson, C., and Pessoa-Brandão, L.: 'Nature, incidence, and cause of work-related amputations in Minnesota', American journal of industrial medicine, 2000, 37, (5), pp. 542-550

10 Owings, M., and Kozak, L.J.: 'Ambulatory and inpatient procedures in the United States, 1996', 1998

11 Ziegler-Graham, K., MacKenzie, E.J., Ephraim, P.L., Travison, T.G., and Brookmeyer, R.: 'Estimating the prevalence of limb loss in the United States: 2005 to 2050', Archives of physical medicine and rehabilitation, 2008, 89, (3), pp. 422-429

12 https://advancedamputees.com/amputee-statistics-you-ought-know 
13 Popovic, D., Tomovic, R., Tepavac, D., and Schwirtlich, L.: 'Control aspects of active above-knee prosthesis', International journal of man-machine studies, 1991, 35, (6), pp. 751-767

14 Bédard, S., and Roy, P.-O.: 'Actuated leg prosthesis for above-knee amputees', in Editor (Ed.)^(Eds.): 'Book Actuated leg prosthesis for above-knee amputees' (Google Patents, 2008, edn.), pp.

15 Herr, H.M., and Grabowski, A.M.: 'Bionic ankle-foot prosthesis normalizes walking gait for persons with leg amputation', Proceedings of the Royal Society B: Biological Sciences, 2011, 279, (1728), pp. 457-464

16 Holmberg, W.S.U.: 'An autonomous control system for a prosthetic foot ankle', IFAC Proceedings Volumes, 2006, 39, (16), pp. 856-861

17 Liu, M., Datseris, P., and Huang, H.H.: 'A prototype for smart prosthetic legsanalysis and mechanical design', in Editor (Ed.)^(Eds.): 'Book A prototype for smart prosthetic legs-analysis and mechanical design' (Trans Tech Publ, 2012, edn.), pp. 19992006

18 Sup, F., Bohara, A., and Goldfarb, M.: 'Design and control of a powered transfemoral prosthesis', The International journal of robotics research, 2008, 27, (2), pp. 263-273

19 Au, S.K., Herr, H., Weber, J., and Martinez-Villalpando, E.C.: 'Powered ankle-foot prosthesis for the improvement of amputee ambulation', in Editor (Ed.)^(Eds.): 'Book Powered ankle-foot prosthesis for the improvement of amputee ambulation' (IEEE, 2007, edn.), pp. 3020-3026

20 Klute, G., Czerniecki, J., and Hannaford, B.: 'Muscle-like pneumatic actuators for below-knee prostheses', in Editor (Ed.)^(Eds.): 'Book Muscle-like pneumatic actuators for below-knee prostheses’ (2000, edn.), pp. 289-292

21 Koniuk, W.: 'Self-adjusting prosthetic ankle apparatus', in Editor (Ed.)^(Eds.): 'Book Self-adjusting prosthetic ankle apparatus' (Google Patents, 2002, edn.), pp.

22 Li, C., Tokuda, M., Furusho, J., Koyanagi, K.i., Morimoto, S., Hashimoto, Y., Nakagawa, A., and Akazawa, Y.: 'Research and development of the intelligentlycontrolled prosthetic ankle joint', in Editor (Ed.)^(Eds.): 'Book Research and development of the intelligently-controlled prosthetic ankle joint' (IEEE, 2006, edn.), pp. 1114-1119

23 Williams, R.J., Hansen, A.H., and Gard, S.A.: 'Prosthetic ankle-foot mechanism capable of automatic adaptation to the walking surface', Journal of biomechanical engineering, 2009, 131, (3), pp. 035002

24 Hansen, A., Gard, S., Childress, D., Ruhe, B., and Williams, R.: 'Automaticallyadapting ankle-foot prosthesis concept', in Editor (Ed.) $)^{\wedge}$ Eds.): 'Book Automaticallyadapting ankle-foot prosthesis concept' (2007, edn.), pp. 
25 Collins, S.H., and Kuo, A.D.: 'Controlled energy storage and return prosthesis reduces metabolic cost of walking', Power, 2005, 600, pp. 800

26 Lawson, B.E., Mitchell, J., Truex, D., Shultz, A., Ledoux, E., and Goldfarb, M.: ‘A robotic leg prosthesis: Design, control, and implementation', IEEE Robotics \& Automation Magazine, 2014, 21, (4), pp. 70-81

27 Bellman, R.D., Holgate, M.A., and Sugar, T.G.: 'SPARKy 3: Design of an active robotic ankle prosthesis with two actuated degrees of freedom using regenerative kinetics', in Editor (Ed.)^(Eds.): 'Book SPARKy 3: Design of an active robotic ankle prosthesis with two actuated degrees of freedom using regenerative kinetics' (IEEE, 2008, edn.), pp. 511516

28 Au, S.K., Bonato, P., and Herr, H.: 'An EMG-position controlled system for an active ankle-foot prosthesis: an initial experimental study', in Editor (Ed.)^(Eds.): 'Book An EMG-position controlled system for an active ankle-foot prosthesis: an initial experimental study' (IEEE, 2005, edn.), pp. 375-379

29 Au, S.K., Weber, J., and Herr, H.: 'Biomechanical design of a powered ankle-foot prosthesis', in Editor (Ed.)^(Eds.): 'Book Biomechanical design of a powered ankle-foot prosthesis' (IEEE, 2007, edn.), pp. 298-303

$30 \mathrm{Au}$, S.K., Weber, J., and Herr, H.: 'Powered ankle--foot prosthesis improves walking metabolic economy', IEEE Transactions on Robotics, 2009, 25, (1), pp. 51-66

31 https://en.wikipedia.org/wiki/Body_area_network

32 Ullah, S., Higgins, H., Braem, B., Latre, B., Blondia, C., Moerman, I., Saleem, S., Rahman, Z., and Kwak, K.S.: 'A comprehensive survey of wireless body area networks', Journal of medical systems, 2012, 36, (3), pp. 1065-1094

33 Chen, M., Gonzalez, S., Vasilakos, A., Cao, H., and Leung, V.C.: 'Body area networks: A survey', Mobile networks and applications, 2011, 16, (2), pp. 171-193

34 Movassaghi, S., Abolhasan, M., Lipman, J., Smith, D., and Jamalipour, A.: 'Wireless body area networks: A survey', IEEE Communications surveys \& tutorials, 2014, 16, (3), pp. 1658-1686

35 Rangayyan, R.M.: 'Biomedical signal analysis' (John Wiley \& Sons, 2015. 2015)

36 Konrad, P.: 'The abc of emg', A practical introduction to kinesiological electromyography, 2005, 1, (2005), pp. 30-35

37 Freriks, B., and Hermens, H.: 'European recommendations for surface electromyography: results of the SENIAM project' (Roessingh Research and Development, 2000. 2000) 
40 Wang, W.-W., and Fu, L.-C.: 'Mirror therapy with an exoskeleton upper-limb robot based on IMU measurement system', in Editor (Ed.)^(Eds.): 'Book Mirror therapy with an exoskeleton upper-limb robot based on IMU measurement system' (IEEE, 2011, edn.), pp. $370-375$

41 Cifuentes, C., Braidot, A., Rodríguez, L., Frisoli, M., Santiago, A., and Frizera, A.: 'Development of a wearable ZigBee sensor system for upper limb rehabilitation robotics', in Editor (Ed.)^(Eds.): 'Book Development of a wearable ZigBee sensor system for upper limb rehabilitation robotics' (IEEE, 2012, edn.), pp. 1989-1994

42 Kim, J.-N., Ryu, M.-H., Yang, Y.-S., and Kim, T.-K.: 'Upper extremity rehabilitation program using inertial sensors and virtual reality for patients with upper extremity hemiplegia due to disorders after stroke’, in Editor (Ed.)^(Eds.): 'Book Upper extremity rehabilitation program using inertial sensors and virtual reality for patients with upper extremity hemiplegia due to disorders after stroke' (2012, edn.), pp. 71-76

43 Eriksson, J., Mataric, M.J., and Winstein, C.J.: 'Hands-off assistive robotics for post-stroke arm rehabilitation', in Editor (Ed.)^(Eds.): 'Book Hands-off assistive robotics for post-stroke arm rehabilitation' (IEEE, 2005, edn.), pp. 21-24

44 Ding, Z., Luo, Z., Causo, A., Chen, I., Yue, K., Yeo, S., and Ling, K.: 'Inertia sensor-based guidance system for upperlimb posture correction', Medical engineering \& physics, 2013, 35, (2), pp. 269-276

45 Fenu, G., and Steri, G.: 'IMU based post-traumatic rehabilitation assessment', in Editor (Ed.)^(Eds.): 'Book IMU based post-traumatic rehabilitation assessment' (IEEE, 2010, edn.), pp. 1-5

46 Yi, J., Zhang, J., Song, D., and Jayasuriya, S.: 'IMU-based localization and slip estimation for skid-steered mobile robots', in Editor (Ed.)^(Eds.): 'Book IMU-based localization and slip estimation for skid-steered mobile robots' (IEEE, 2007, edn.), pp. $2845-2850$

47 Yi, J., Wang, H., Zhang, J., Song, D., Jayasuriya, S., and Liu, J.: 'Kinematic modeling and analysis of skid-steered mobile robots with applications to low-cost inertialmeasurement-unit-based motion estimation', IEEE transactions on robotics, 2009, 25, (5), pp. 1087-1097

48 Ward, C.C., and Iagnemma, K.: 'Model-based wheel slip detection for outdoor mobile robots', in Editor (Ed.)^(Eds.): 'Book Model-based wheel slip detection for outdoor mobile robots' (IEEE, 2007, edn.), pp. 2724-2729 
49 Bloesch, M., Hutter, M., Hoepflinger, M.A., Leutenegger, S., Gehring, C., Remy, C.D., and Siegwart, R.: 'State estimation for legged robots-consistent fusion of leg kinematics and IMU', Robotics, 2013, 17, pp. 17-24

50 Bruggemann, T.S., Greer, D.G., and Walker, R.A.: 'GPS fault detection with IMU and aircraft dynamics', IEEE Transactions on Aerospace and Electronic Systems, 2011, 47, (1), pp. 305-316

51 Allerton, D.J., and Jia, H.: 'A review of multisensor fusion methodologies for aircraft navigation systems', The Journal of Navigation, 2005, 58, (3), pp. 405-417

52 Azfar, A.Z., and Hazry, D.: 'A simple approach on implementing imu sensor fusion in pid controller for stabilizing quadrotor flight control', in Editor (Ed. $)^{\wedge}($ Eds. $)$ : 'Book A simple approach on implementing imu sensor fusion in pid controller for stabilizing quadrotor flight control' (IEEE, 2011, edn.), pp. 28-32

53 Ahmad, N., Ghazilla, R.A.R., Khairi, N.M., and Kasi, V.: 'Reviews on various inertial measurement unit (IMU) sensor applications', International Journal of Signal Processing Systems, 2013, 1, (2), pp. 256-262

54 Kong, K., and Tomizuka, M.: 'A gait monitoring system based on air pressure sensors embedded in a shoe', IEEE/ASME Transactions on mechatronics, 2009, 14, (3), pp. $358-370$

55 Loudon, J.K., Swift, M., and Bell, S.: 'The clinical orthopedic assessment guide' (Human Kinetics, 2008. 2008)

56 Iosa, M., Fusco, A., Marchetti, F., Morone, G., Caltagirone, C., Paolucci, S., and Peppe, A.: 'The golden ratio of gait harmony: repetitive proportions of repetitive gait phases', BioMed research international, 2013, 2013

57 Mannini, A., and Sabatini, A.M.: 'Gait phase detection and discrimination between walking-jogging activities using hidden Markov models applied to foot motion data from a gyroscope', Gait \& posture, 2012, 36, (4), pp. 657-661

58 Kong, K., and Tomizuka, M.: 'Smooth and continuous human gait phase detection based on foot pressure patterns', in Editor $(\mathrm{Ed} .)^{\wedge}(\mathrm{Eds}$.$) : 'Book Smooth and continuous$ human gait phase detection based on foot pressure patterns' (IEEE, 2008, edn.), pp. 36783683

59 Wang, Q., Chai, X., Wang, Y., Liu, D., Chen, M., Li, Y., Liu, X., and Bai, O.: 'A high data rate, multi-nodes wireless personal-area sensor network for real-time data acquisition and control', in Editor (Ed.) ${ }^{\wedge}(E d s$.$) : 'Book A high data rate, multi-nodes$ wireless personal-area sensor network for real-time data acquisition and control' (IEEE, 2017, edn.), pp. 1-5

60 Bai, O., Kelly, G., Fei, D.-Y., Murphy, D., Fox, J., Burkhardt, B., Lovegreen, W., and Soars, J.: 'A wireless, smart EEG system for volitional control of lower-limb 
prosthesis', in Editor (Ed.)^(Eds.): 'Book A wireless, smart EEG system for volitional control of lower-limb prosthesis’ (IEEE, 2015, edn.), pp. 1-6

61 Wang, Y., Zheng, Y., Bai, O., Wang, Q., Liu, D., Liu, X., and Sun, J.: 'A multifunctional wireless body area sensors network with real time embedded data analysis', in Editor (Ed.)^(Eds.): 'Book A multifunctional wireless body area sensors network with real time embedded data analysis' (IEEE, 2016, edn.), pp. 508-511

62 InvenSense, T.: 'MPU-9250, Nine-Axis (Gyro+ Accelerometer+ Compass) MEMS MotionTracking ${ }^{\mathrm{TM}}$ Device', in Editor (Ed.)^(Eds.): 'Book MPU-9250, Nine-Axis (Gyro+ Accelerometer+ Compass) MEMS MotionTrackingTM Device’ (2014, edn.), pp.

63 https://www.tekscan.com/products-solutions/force-sensors/a301

64 https://www.st.com/en/imaging-and-photonics-solutions/vl5310x.html

65 Stepien, J.M., Cavenett, S., Taylor, L., and Crotty, M.: 'Activity levels among lower-limb amputees: self-report versus step activity monitor', Archives of physical medicine and rehabilitation, 2007, 88, (7), pp. 896-900

66 Au, S.K., Bonato, P., and Herr, H.: 'An EMG-position controlled system for an active ankle-foot prosthesis: an initial experimental study', in Editor (Ed.)^(Eds.): 'Book An EMG-position controlled system for an active ankle-foot prosthesis: an initial experimental study' (IEEE, 2005, edn.), pp. 375-379

67 Fey, N.P., Klute, G.K., and Neptune, R.R.: 'Altering prosthetic foot stiffness influences foot and muscle function during below-knee amputee walking: a modeling and simulation analysis', Journal of biomechanics, 2013, 46, (4), pp. 637-644

68 Fey, N.P., Klute, G.K., and Neptune, R.R.: 'Optimization of prosthetic foot stiffness to reduce metabolic cost and intact knee loading during below-knee amputee walking: a theoretical study', Journal of biomechanical engineering, 2012, 134, (11), pp. 111005

69 Childers, W.L., Kistenberg, R.S., and Gregor, R.J.: 'Pedaling asymmetries in cyclists with unilateral transtibial amputation: effect of prosthetic foot stiffness', Journal of applied biomechanics, 2011, 27, (4), pp. 314-321

70 Fey, N.P., Klute, G.K., and Neptune, R.R.: 'The influence of energy storage and return foot stiffness on walking mechanics and muscle activity in below-knee amputees', Clinical Biomechanics, 2011, 26, (10), pp. 1025-1032

71 Robert Gailey PhD, P.: 'Review of secondary physical conditions associated with lower-limb amputation and long-term prosthesis use', Journal of rehabilitation research and development, 2008, 45, (1), pp. 15 
72 Sewell, P., Noroozi, S., Vinney, J., and Andrews, S.: 'Developments in the transtibial prosthetic socket fitting process: A review of past and present research', Prosthetics and Orthotics International, 2000, 24, (2), pp. 97-107

73 Hanspal, R., Fisher, K., and Nieveen, R.: 'Prosthetic socket fit comfort score', Disability and rehabilitation, 2003, 25, (22), pp. 1278-1280

74 Wang, D., Liu, M., Zhang, F., and Huang, H.: 'Design of an expert system to automatically calibrate impedance control for powered knee prostheses', in Editor (Ed.) $)^{\wedge}$ (Eds.): 'Book Design of an expert system to automatically calibrate impedance control for powered knee prostheses' (IEEE, 2013, edn.), pp. 1-5

75 Huang, H., Crouch, D.L., Liu, M., Sawicki, G.S., and Wang, D.: 'A cyber expert system for auto-tuning powered prosthesis impedance control parameters', Annals of biomedical engineering, 2016, 44, (5), pp. 1613-1624

76 Wen, Y., Brandt, A., Liu, M., Huang, H.H., and Si, J.: 'Comparing parallel and sequential control parameter tuning for a powered knee prosthesis', in Editor (Ed.) $)^{\wedge}$ (Eds.): 'Book Comparing parallel and sequential control parameter tuning for a powered knee prosthesis' (IEEE, 2017, edn.), pp. 1716-1721

77 Cain, S.M., Gordon, K.E., and Ferris, D.P.: 'Locomotor adaptation to a powered ankle-foot orthosis depends on control method', Journal of neuroengineering and rehabilitation, 2007, 4, (1), pp. 48

78 Sawicki, G.S., and Ferris, D.P.: 'Mechanics and energetics of level walking with powered ankle exoskeletons', Journal of Experimental Biology, 2008, 211, (9), pp. 14021413

79 Ferris, D.P., Gordon, K.E., Sawicki, G.S., and Peethambaran, A.: 'An improved powered ankle-foot orthosis using proportional myoelectric control', Gait \& posture, 2006, 23, (4), pp. 425-428

80 Atri, R., Marquez, J.S., Murphy, D., Gorgey, A., Fei, D., Fox, J., Burkhardt, B., Lovegreen, W., and Bai, O.: 'Investigation of muscle activity during loaded human gait using signal processing of multi-channel surface EMG and IMU', in Editor (Ed.)^(Eds.): 'Book Investigation of muscle activity during loaded human gait using signal processing of multi-channel surface EMG and IMU' (IEEE, 2016, edn.), pp. 1-6

81 Atri, R., Marquez, J.S., Murphy, D., Gorgey, A., Fei, D., Fox, J., Lovegreen, W., and Bai, O.: 'EMG-based energy expenditure optimization for active prosthetic leg tuning', in Editor (Ed.)^(Eds.): 'Book EMG-based energy expenditure optimization for active prosthetic leg tuning' (IEEE, 2017, edn.), pp. 394-397

82 Kumar, M., Husian, M., Upreti, N., and Gupta, D.: 'Genetic algorithm: Review and application', International Journal of Information Technology and Knowledge Management, 2010, 2, (2), pp. 451-454 
83 Whitley, D.: 'A genetic algorithm tutorial', Statistics and computing, 1994, 4, (2), pp. $65-85$

84 Chelouah, R., and Siarry, P.: 'Genetic and Nelder-Mead algorithms hybridized for a more accurate global optimization of continuous multiminima functions', European Journal of Operational Research, 2003, 148, (2), pp. 335-348

85 Iman, R.L.: 'Latin hypercube sampling', Wiley StatsRef: Statistics Reference Online, 2008

86 Konrad, P.: 'A Practical Introduction to Kinesiological Electromyography, Noraxon INC', in Editor (Ed.)^(Eds.): 'Book A Practical Introduction to Kinesiological Electromyography, Noraxon INC' (USA, 2005, edn.), pp.

87 Merletti, R., and Parker, P.A.: 'Electromyography: physiology, engineering, and non-invasive applications' (John Wiley \& Sons, 2004. 2004)

88 Hermens, H.J., Freriks, B., Merletti, R., Stegeman, D., Blok, J., Rau, G., Disselhorst-Klug, C., and Hägg, G.: 'European recommendations for surface electromyography', Roessingh research and development, 1999, 8, (2), pp. 13-54

89 Herr, H.M., Han, Z., Barnhart, C.E., and Casler Jr, R.J.: 'Prosthetic, orthotic or exoskeleton device', in Editor (Ed.)^(Eds.): 'Book Prosthetic, orthotic or exoskeleton device' (Google Patents, 2015, edn.), pp.

90 Konrad, P.: 'The abc of emg', A practical introduction to kinesiological electromyography, 2005, 1, pp. 30-35

91 De Luca, C.J.: 'The use of surface electromyography in biomechanics', Journal of applied biomechanics, 1997, 13, (2), pp. 135-163

92 Moritani, T., and Muro, M.: 'Motor unit activity and surface electromyogram power spectrum during increasing force of contraction', European journal of applied physiology and occupational physiology, 1987, 56, (3), pp. 260-265

93 Yana, K., Mizuta, H., and Kajiyama, R.: 'Surface electromyogram recruitment analysis using higher order spectrum', in Editor $(E d .)^{\wedge}(E d s$.$) : 'Book Surface$ electromyogram recruitment analysis using higher order spectrum' (IEEE, 1995, edn.), pp. $1345-1346$

94 Reaz, M.B.I., Hussain, M., and Mohd-Yasin, F.: 'Techniques of EMG signal analysis: detection, processing, classification and applications', Biological procedures online, 2006, 8, (1), pp. 11

95 Umberger, B.R., Gerritsen, K.G., and Martin, P.E.: 'A model of human muscle energy expenditure', Computer methods in biomechanics and biomedical engineering, 2003, 6, (2), pp. 99-111 
96 Lagarias, J.C., Reeds, J.A., Wright, M.H., and Wright, P.E.: 'Convergence properties of the Nelder--Mead simplex method in low dimensions', SIAM Journal on optimization, 1998, 9, (1), pp. 112-147

97 Gao, F., and Han, L.: 'Implementing the Nelder-Mead simplex algorithm with adaptive parameters', Computational Optimization and Applications, 2012, 51, (1), pp. 259-277

98 Singer, S., and Nelder, J.: 'Nelder-mead algorithm', Scholarpedia, 2009, 4, (7), pp. 2928

99 Hastings, W.K.: 'Monte Carlo sampling methods using Markov chains and their applications', Biometrika, 1970, 57, (1), pp. 97-109

100 Helton, J.C., and Davis, F.J.: 'Latin hypercube sampling and the propagation of uncertainty in analyses of complex systems', Reliability Engineering \& System Safety, 2003, 81, (1), pp. 23-69

101 Ye, T., and Kalyanaraman, S.: 'A recursive random search algorithm for large-scale network parameter configuration', ACM SIGMETRICS Performance Evaluation Review, 2003, 31, (1), pp. 196-205

102 McKay, M.D., Beckman, R.J., and Conover, W.J.: 'A comparison of three methods for selecting values of input variables in the analysis of output from a computer code', Technometrics, 2000, 42, (1), pp. 55-61

103 Olsson, A.M., and Sandberg, G.E.: 'Latin hypercube sampling for stochastic finite element analysis', Journal of Engineering Mechanics, 2002, 128, (1), pp. 121-125

104 Owen, A.B.: 'Controlling correlations in Latin hypercube samples', Journal of the American Statistical Association, 1994, 89, (428), pp. 1517-1522

105 McKay, M.D., Beckman, R.J., and Conover, W.J.: 'Comparison of three methods for selecting values of input variables in the analysis of output from a computer code', Technometrics, 1979, 21, (2), pp. 239-245

106 Tucker, M.R., Olivier, J., Pagel, A., Bleuler, H., Bouri, M., Lambercy, O., del R Millán, J., Riener, R., Vallery, H., and Gassert, R.: 'Control strategies for active lower extremity prosthetics and orthotics: a review', Journal of neuroengineering and rehabilitation, 2015, 12, (1), pp. 1

107 Martinez-Villalpando, E.C., Mooney, L., Elliott, G., and Herr, H.: 'Antagonistic active knee prosthesis. A metabolic cost of walking comparison with a variable-damping prosthetic knee', in Editor $(E d .)^{\wedge}(E d s$.$) : 'Book Antagonistic active knee prosthesis. A$ metabolic cost of walking comparison with a variable-damping prosthetic knee' (IEEE, 2011, edn.), pp. 8519-8522 
108 Farris, R.J., Quintero, H.A., Murray, S.A., Ha, K.H., Hartigan, C., and Goldfarb, M.: 'A preliminary assessment of legged mobility provided by a lower limb exoskeleton for persons with paraplegia', IEEE Transactions on neural systems and rehabilitation engineering, 2013, 22, (3), pp. 482-490

109 Jin, D., Yang, J., Zhang, R., Wang, R., and Zhang, J.: 'Terrain identification for prosthetic knees based on electromyographic signal features', Tsinghua Science and Technology, 2006, 11, (1), pp. 74-79

110 Huang, H., Kuiken, T.A., and Lipschutz, R.D.: 'A strategy for identifying locomotion modes using surface electromyography', IEEE Transactions on Biomedical Engineering, 2009, 56, (1), pp. 65-73

111 Huang, H., Zhang, F., Hargrove, L.J., Dou, Z., Rogers, D.R., and Englehart, K.B.: 'Continuous locomotion-mode identification for prosthetic legs based on neuromuscularmechanical fusion', IEEE Transactions on Biomedical Engineering, 2011, 58, (10), pp. 2867-2875

112 Varol, H.A., Sup, F., and Goldfarb, M.: 'Multiclass real-time intent recognition of a powered lower limb prosthesis', IEEE Transactions on Biomedical Engineering, 2010, 57, (3), pp. 542-551

113 Hargrove, L.J., Simon, A.M., Young, A.J., Lipschutz, R.D., Finucane, S.B., Smith, D.G., and Kuiken, T.A.: 'Robotic leg control with EMG decoding in an amputee with nerve transfers', New England Journal of Medicine, 2013, 369, (13), pp. 1237-1242

114 Wentink, E., Beijen, S., Hermens, H.J., Rietman, J.S., and Veltink, P.H.: 'Intention detection of gait initiation using EMG and kinematic data', Gait \& posture, 2013, 37, (2), pp. $223-228$

115 Hoover, C.D., Fulk, G.D., and Fite, K.B.: 'Stair ascent with a powered transfemoral prosthesis under direct myoelectric control', IEEE/ASME Transactions on Mechatronics, 2013, 18, (3), pp. 1191-1200

116 Spanias, J.A., Perreault, E.J., and Hargrove, L.J.: 'Detection of and compensation for EMG disturbances for powered lower limb prosthesis control', IEEE Transactions on Neural Systems and Rehabilitation Engineering, 2016, 24, (2), pp. 226-234

117 Spanias, J., Simon, A., Finucane, S., Perreault, E., and Hargrove, L.: 'Online adaptive neural control of a robotic lower limb prosthesis', Journal of neural engineering, 2018, 15, (1), pp. 016015

118 Stegeman, D., and Hermens, H.: 'Standards for surface electromyography: The European project Surface EMG for non-invasive assessment of muscles (SENIAM)', Enschede: Roessingh Research and Development, 2007, pp. 108-112 
120 Safavian, S.R., and Landgrebe, D.: 'A survey of decision tree classifier methodology', IEEE transactions on systems, man, and cybernetics, 1991, 21, (3), pp. 660674

121 Désir, C., Petitjean, C., Heutte, L., Salaun, M., and Thiberville, L.: 'Classification of endomicroscopic images of the lung based on random subwindows and extra-trees', IEEE Transactions on Biomedical Engineering, 2012, 59, (9), pp. 2677-2683

122 Vapnik, V.: 'The nature of statistical learning theory' (Springer science \& business media, 2013. 2013)

123 Suykens, J.A., and Vandewalle, J.: 'Least squares support vector machine classifiers', Neural processing letters, 1999, 9, (3), pp. 293-300

124 Kannathal, N., Acharya, U.R., Lim, C.M., Sadasivan, P., and Krishnan, S.: 'Classification of cardiac patient states using artificial neural networks', Experimental \& Clinical Cardiology, 2003, 8, (4), pp. 206

125 Manda, A., Walker, R.B., and Khamanga, S.M.: 'An Artificial Neural Network Approach to Predict the Effects of Formulation and Process Variables on Prednisone Release from a Multipartite System', Pharmaceutics, 2019, 11, (3), pp. 109

126 Malouf, R.: 'A comparison of algorithms for maximum entropy parameter estimation', in Editor (Ed.)^(Eds.): 'Book A comparison of algorithms for maximum entropy parameter estimation' (Association for Computational Linguistics, 2002, edn.), pp. $1-7$

127 Andrew, G., and Gao, J.: 'Scalable training of L 1-regularized log-linear models', in Editor (Ed.) $)^{\wedge}$ Eds.): 'Book Scalable training of L 1-regularized log-linear models' (ACM, 2007, edn.), pp. 33-40 
VITA

ROOZBEH ATRI

2007-2012

B.Sc., Electrical and Electronic Engineering University of Tabriz

Tabriz, Iran

2012-2015

M.Sc., Bio-Electrical Engineering K. N. Toosi University of Technology

Tehran, Iran

2016-2019

$\mathrm{PhD}$, Electrical and Computer Engineering

Florida International University

Miami, Florida, USA

\section{PUBLICATIONS}

1. Atri, R., Marquez, J., Leung, C., Siddiquee, M., Murphy, D., Gorgey, A., Lovegreen, W., Fei, D.Y. and Bai, O., 2018. Smart Data-Driven Optimization of Powered Prosthetic Ankles Using Surface Electromyography. Sensors, 18(8), p.2705.

2. Marquez, J.S., Atri, R. and Bai, O., 2018, July. Exploration of Metrics for Leg Length Discrepancy Using a Wearable Gait Analysis System. In 2018 40th Annual International Conference of the IEEE Engineering in Medicine and Biology Society (EMBC) (pp. 1-4). IEEE.

3. Siddiquee, M.R., Marquez, J.S., Atri, R., Ramon, R., Mayrand, R.P. and Bai, O., 2018. Movement artefact removal from NIRS signal using multi-channel IMU data. BioMedical Engineering OnLine, 17(1), p.120.

4. Atri, R., Marquez, J.S., Murphy, D., Gorgey, A., Fei, D., Fox, J., Burkhardt, B., Lovegreen, W. and Bai, O., 2016, December. Investigation of muscle activity during loaded human gait using signal processing of multi-channel surface EMG and IMU. In Signal Processing in Medicine and Biology Symposium (SPMB), 2016 IEEE (pp. 16). IEEE.

5. Bai, O., Atri, R., Marquez, J.S. and Fei, D.Y., 2017, March. Characterization of lower limb activity during gait using wearable, multi-channel surface EMG and IMU sensors. In Electrical Engineering Congress (iEECON), 2017 International (pp. 1-4). IEEE.

6. Atri, R., Marquez, J.S., Murphy, D., Gorgey, A., Fei, D., Fox, J., Lovegreen, W. and Bai, O., 2017, July. EMG-based energy expenditure optimization for active prosthetic 
leg tuning. In Engineering in Medicine and Biology Society (EMBC), 2017 39th Annual International Conference of the IEEE (pp. 394-397). IEEE.

7. Murphy, D.P., Bai, O., Gorgey, A.S., Fox, J., Lovegreen, W.T., Burkhardt, B.W., Atri, R., Marquez, J.S., Li, Q. and Fei, D.Y., 2017. Electroencephalogram-Based Braincomputer interface and Lower-Limb Prosthesis control: A case study. Frontiers in neurology, 8, p.696.

8. Atri, R. and Mohebbi, M., 2014. Screening Obstructive Sleep Apnea Using Wavelet Features of ECG Signals. In Biomedical Engineering (ICBME), 2014. 6th Iranian conference on E-health and ICT application in Medical science.

9. Atri, R., Marquez, J., Murphy, D., Lovegreen, W., Gorgey, A., Fei, D.Y. and Bai, O., 2019. Prediction of Locomotion Mode Transitions Using Neural and Kinematic Signals for Powered Prosthetic Control. Submitted In Medical \& Biological Engineering \& Computing.

10. Siddiquee, M.R., Atri, R., Marquez, J.S., Shafiul, H., Ramon, R., Mayrand, R.P. and Bai, O., 2019. Sensor Location Optimization of Wireless Wearable fNIRS System for Cognitive Workload Monitoring Using a Data Driven Approach for Improved Wearability. Submitted In PLOS One. 